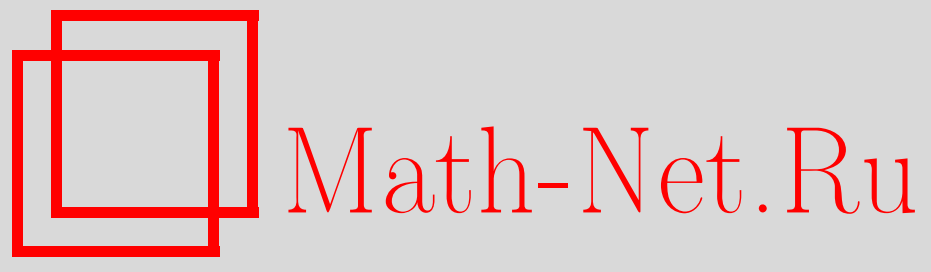

И. Д. Шкредов, Структурные теоремы в аддитивной комбинаторике, УМH, 2015, том 70, выпуск 1, 123-178

DOI: https://doi.org/10.4213/rm9647

Использование Общероссийского математического портала Math-Net.Ru подразумевает, что вы прочитали и согласны с пользовательским соглашением http://www . mathnet.ru/rus/agreement

Параметры загрузки:

IP: 18.234 .197 .8

26 апреля 2023 г., 05:15:39

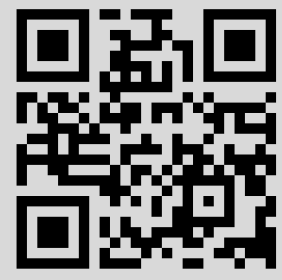




\section{Структурные теоремы в аддитивной комбинаторике}

\section{И. Д. Шкредов}

В обзоре рассматривается несколько структурных результатов аддитивной комбинаторики. Обсуждаются классические и современные проблемы комбинаторной теории чисел, анализ Фурье высших порядков, обратные теоремы для норм Гауэрса, старшие энергии, связь между комбинаторной и аналитической теорией чисел.

Библиография: 149 названий.

Ключевые слова: аддитивная комбинаторика, множества с малым удвоением, нормы Гауэрса, анализ Фурье.

DOI: $10.4213 / \mathrm{rm} 9647$

\section{СоДЕРЖАНИЕ}

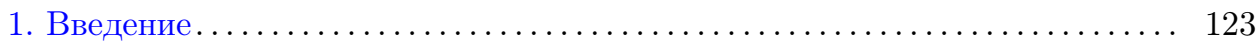

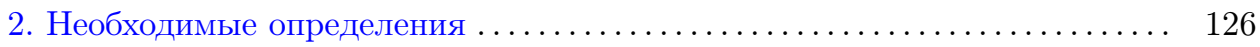

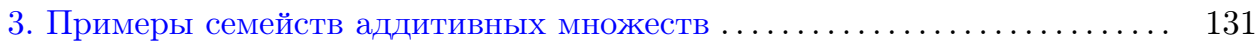

4. Строение множеств с малым удвоением..................... 135

5. Доказательство теоремы Фреймана и теоремы Сандерса в группе $\mathbb{F}_{2}^{n} \ldots 142$

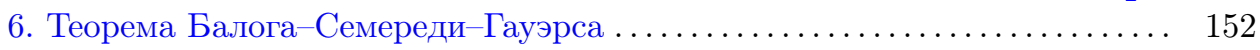

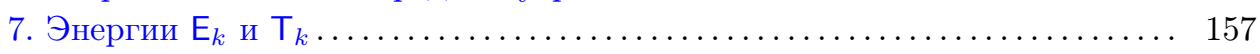

8. Структурные теоремы для норм Гауэрса...................... 163

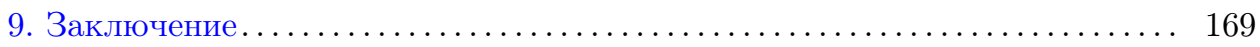

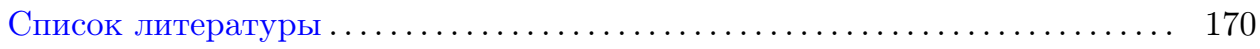

\section{1. Введение}

Аддитивная комбинаторика - это промежуточный между комбинаторикой и теорией чисел раздел математики, в котором изучают комбинаторные вопросы, связанные с групповой операцией (см. книги [1], [2] и обзоры [3], [4]). Иными словами, основным предметом данной науки является комбинаторика подмножеств некоторой группы $\mathbf{G}$, а специфические алгебраические свойства

Исследование выполнено за счет гранта Российского научного фонда (проект 14-11-00433). 
“обертывающей” группы G обычно оставляются без внимания. Если изучается комбинаторика подмножеств данного кольца, то говорят об арифметической комбинаторике.

Настоящий обзор посвящен одной важной особенности аддитивной комбинаторики, которую, несколько расплывчато, можно сформулировать как принцип существования жесткой структуры при наличии какого-либо "критического", соотношения. Мы имеем в виду следующую абстрактную постановку. Предположим, что у нас есть некоторое семейство объектов и действительный функционал на нем. Пусть также известно, что максимальное значение этого функционала равно $M$. Возьмем (не очень большой) параметр $K \geqslant 1$ и зададим такой вопрос: что можно сказать об объектах нашего семейства со значением функционала, большим $M / K$ ? Есть ли у этих объектов какая-то структура? Наоборот, гарантирует ли наличие у объекта подобной структуры то, что значение нашего функционала на нем будет большим? Замечательно, что в широком классе аддитивно-комбинаторных задач ответы на эти вопросы оказываются положительными, и искомые "экстремальные" подобъекты чрезвычайно структурированы. Аналогичные идеи обсуждаются в обзорах [5] и [6].

Приведем два примера этого общего феномена, первый - из простейшей теории функций, а второй - собственно из аддитивной комбинаторики. Пусть у нас есть действительные функции $f$ на отрезке $\{1,2, \ldots, n\}$ с ненормированной $L_{2}$-нормой, равной $n^{1 / 2}$, иными словами, пусть для каждой такой функции выполнено равенство $\sum_{x=1}^{n}|f(x)|^{2}=n$. Тогда по неравенству Коши-Буняковского $L_{1}$-норма любой функции $f$ не превосходит $n$. Это глобальная верхняя оценка для семейства функций с $L_{2}$-нормой, равной $n^{1 / 2}$, а функционалом на данном семействе выступает $L_{1}$-норма. Что можно сказать о строении функций, для которых выполнено неравенство $\|f\|_{L_{1}} \geqslant n / K$ ? Легко видеть, что у таких функций много больших значений. Действительно, пусть

$$
\Omega_{K}=\Omega_{K}(f)=\left\{x \in\{1,2, \ldots, n\}:|f(x)| \geqslant \frac{1}{2 K}\right\} .
$$

Тогда $\sum_{x \in \Omega_{K}}|f(x)| \geqslant \frac{n}{2 K}$, и, снова применяя неравенство Коши-Буняковского, а также условие $\sum_{x=1}^{n}|f(x)|^{2}=n$, мы имеем $\left|\Omega_{K}\right| \geqslant \frac{n}{4 K^{2}}$. Таким образом, если параметр $K$ мал, то мощность множества $\Omega_{K}$ сравнима с длиной $n$ всего отрезка. Наоборот, если для функции $f$ выполнено неравенство $\left|\Omega_{K}(f)\right| \geqslant n /\left(4 K^{2}\right)$, то, как легко видеть, ее $L_{1}$-норма не меньше, чем $n /\left(8 K^{3}\right)$. Используя обозначение $p_{j}(K)$ для многочленов от $K$, мы получаем, что для нашего семейства функций условие $\|f\|_{L_{1}} \geqslant n / p_{1}(K)$ выполнено тогда и только тогда, когда $\left|\Omega_{p_{2}(K)}(f)\right| \geqslant n / p_{3}(K)$. Таким образом, в некотором грубом смысле, характеризация через мощность множества $\Omega_{K}$ является критерием для функции иметь большую $L_{1}$-норму.

Во втором примере в качестве объектов мы рассматриваем конечные подмножества произвольной абелевой группы, например группы целых чисел, а функционалом выступает отношение мощности множества к мощности множества 
его попарных сумм. Ясно, что это отношение не превосходит единицы. Кроме того, оказывается, что можно полностью описать все множества, у которых рассматриваемое отношение велико. Грубо говоря, эти множества будут очень экономно лежать в суммах небольшого числа арифметических прогрессий (см. раздел 4). В некотором смысле с данного структурного результата [7] и началась современная аддитивная комбинаторика.

В последнее время (см. [8]-[23] и др.) выяснилось, что в очень широком классе аддитивно-комбинаторных задач удается доказать подобные структурные результаты, причем возникающие здесь семейства объектов могут весьма отличаться от классических. Основной целью настоящего обзора является демонстрация недавних достижений в этой области. В каждом разделе настоящей статьи мы формулируем соответствующие структурные теоремы для своих функционалов и семейств, а также стараемся привести несколько приложений полученных результатов.

Опишем вкратце структуру настоящего обзора. В разделе 2 мы напоминаем некоторые понятия аддитивной комбинаторики и анализа Фурье. Следующий раздел 3 содержит несколько примеров семейств аддитивных множеств, с которыми приходится иметь дело в комбинаторной теории чисел. Изложение проходит, в основном, в исторической последовательности. Мы рассматриваем вначале случайные множества (которые с точки зрения аддитивной комбинаторики являются скорее предметом исследования теории вероятностей и теории чисел), затем классическое аддитивно-комбинаторное семейство множеств с малым удвоением и, наконец, новые семейства, ставшие предметом интенсивных исследований последних лет. Более подробно лучше всего изученный предмет аддитивной комбинаторики - множества с малым удвоением - обсуждается в следующем разделе 4 . В разделе 5 мы даем доказательство теоремы Фреймана - классического аддитивно-комбинаторного результата и теоремы Сандерса-Конягина, относящейся к новейшим достижениям в этой области. Доказательства приводятся лишь для группы $\mathbb{F}_{2}^{n}$. Рассуждения в этой группе обладают чрезвычайной наглядностью и практически не содержат технических моментов. Поэтому данная часть обзора является самым простым введением в предмет. Попутно в этом разделе мы приводим понятия спектра и полиномиального роста в группах, а также такие важные инструменты, как, например, неравенство треугольника Ружи и неравенство Плюннеке [2], лемму о почти периодичности Крута-Сисаска [24], [25] и теорему Чанг о коэффициентах Фурье [26]. Далее, в разделе 6 мы обсуждаем важный аддитивно-комбинаторный результат - теорему Балога-Семереди-Гауэрса и иллюстрируем ее применение на примере задачи о суммах произведений в конечном поле. С точки зрения философии, изложенной выше, данная теорема представляет собой результат о структуре множеств с экстремальным соотношением между мощностью и четвертым моментом их преобразования Фурье. Раздел 7 посвящен последним результатам из теории старших энергий сверток и преобразований Фурье характеристических функций множеств. Очень интересно, что экстремальными семействами в подобного рода задачах будут уже не классические множества с малым удвоением, а более сложно устроенные аддитивные множества. В разделе 8 обсуждаются обратные теоремы для норм Гауэрса и вытекающие из них декомпозиционные результаты, а также имеющиеся здесь приложения к теории 
чисел и аддитивной комбинаторике. Наконец, в заключении рассматривается ряд открытых проблем.

При написании этой статьи мы интенсивно пользовались обзорами [4], [27] и лекциями [28].

\section{2. Необходимые определения}

В этом разделе мы напомним некоторые понятия аддитивной комбинаторики, а также анализа Фурье. Пусть $\mathbf{G}=(\mathbf{G},+)$ - локально компактная абелева группа с аддитивной групповой операцией + . Хорошо известно, что на всякой такой группе существует мера Хаара $\mu_{\mathbf{G}}$, т. е. неотрицательная регулярная мера (все определения могут быть найдены, например, в [29; гл. 1, 2 и добавление $E])$ такая, что для всякого борелевского множества $B$ и произвольного элемента $x \in \mathbf{G}$ имеем

$$
\mu_{\mathbf{G}}(B+x)=\mu_{\mathbf{G}}(B) .
$$

На пространстве $L_{2}\left(\mathbf{G}, \mu_{\mathbf{G}}\right)=L_{2}(\mathbf{G})$ задано скалярное произведение двух комплексных функций $f$ и $g$ :

$$
\langle f, g\rangle=\int_{\mathbf{G}} f(x) \overline{g(x)} d \mu_{\mathbf{G}}(x) .
$$

Пусть далее $\widehat{\mathbf{G}}$ - двойственная группа для группы $\mathbf{G}$, т. е. локально компактная абелева группа непрерывных гомоморфизмов $\xi$ из $\mathbf{G}$ в $\mathbf{S}^{1}=\{z \in \mathbb{C}:|z|=1\}$, $\xi: x \mapsto \xi \cdot x=\xi(x), x \in \mathbf{G}$. Преобразование Фурье $(\Phi f)(\xi)=\widehat{f}(\xi)$ произвольной комплексной функции $f \in L_{1}(\mathbf{G})$ задается формулой

$$
(\Phi f)(\xi)=\widehat{f}(\xi)=\int_{\mathbf{G}} f(x) \overline{\xi(x)} d \mu_{\mathbf{G}}(x) .
$$

Ясно, что всегда справедливо неравенство

$$
\|\widehat{f}\|_{\infty} \leqslant \int_{\mathbf{G}}|f(x)| d \mu_{\mathbf{G}}(x)=\|f\|_{1} .
$$

Пусть $\Gamma \subseteq \widehat{\mathbf{G}}-$ некоторое множество характеров и $\varepsilon \in[0,1]$ - действительное число. Определим множество Бора, порожденное множеством Г:

$$
\mathscr{B}(\Gamma, \varepsilon)=\left\{x \in \mathbf{G}:\|\xi \cdot x\| \leqslant \frac{\varepsilon}{2 \pi} \text { для всех } \xi \in \Gamma\right\},
$$

где $\|\cdot\|$ - евклидова метрика на окружности. Можно сказать, что величина $\|\xi \cdot x\|$ измеряет, насколько $\xi$ отличается от тривиального характера $\xi_{0} \equiv 1$.

В дальнейшем нам понадобится понятие свертки двух функций $f, g \in L_{1}(\mathbf{G})$ :

$$
(f * g)(x)=\int_{\mathbf{G}} f(y) g(x-y) d \mu_{\mathbf{G}}(x),
$$

а также другой вариант свертки:

$$
(f \circ g)(x)=\int_{\mathbf{G}} f(y) g(x+y) d \mu_{\mathbf{G}}(x) .
$$


Если $k$ - натуральное число, то $k$-я свертка обозначается $*_{k}$ и определяется по индукции: $*_{k}:=*\left(*_{k-1}\right)$. Имеем (см., например, [29])

$$
\widehat{(f * g)}(\xi)=\widehat{f}(\xi) \widehat{g}(\xi) \quad \text { и } \quad \widehat{(f \circ g)}(\xi)=\overline{\bar{f}} \widehat{g} .
$$

Операция свертки очень важна для аддитивных задач комбинаторной теории чисел. Действительно, пусть $A$ и $B$ - произвольные подмножества группы $\mathbf{G}$. Определим сумму (по Минковскому) этих множеств:

$$
A+B:=\{c \in \mathbf{G}: c=a+b, a \in A, b \in B\} .
$$

Пусть $A(x)$ - характеристическая функция множества $A$, а $B(x)$ - характеристическая функция множества $B$. Легко видеть, что $(A * B)(x) \neq 0$ гарантирует включение $x \in A+B$, а в случае конечной группы $\mathbf{G}$ имеем $\operatorname{supp}(A * B)=A+B$. Свертка о соответствует разности множеств $A$ и $B$ :

$$
A-B:=\{c \in \mathbf{G}: c=a-b, a \in A, b \in B\} .
$$

Аналогично (3) определяется сумма нескольких множеств. Если в группе $(\mathbf{G},+)$ есть еще одна бинарная операция, скажем, умножение, то тогда можно определить и произведение множеств. В дальнейшем нам понадобится $A \dot{+} B-$ сумма различных элементов множеств $A$ и $B$ :

$$
A+B:=\{c \in \mathbf{G}: c=a+b, a \in A, b \in B, a \neq b\} .
$$

Обозначим через $A_{1} \dot{+} A_{2} \dot{+} \cdots \dot{+} A_{d}$ множество, образованное суммой различных элементов из множеств $A_{1}, \ldots, A_{d}$. Множество, состоящее из суммы $d$ различных элементов множества $A$, обозначим через $d \dot{A}$.

Часто мы будем рассматривать ситуацию, когда абелева группа $\mathbf{G}$ конечна. Хорошо известно, что в этом случае $\widehat{\mathbf{G}}=\mathbf{G}$. Обозначим через $N$ мощность группы G. Очень удобно определить преобразование Фурье функции $f$ формулой

$$
(\Phi f)(\xi)=\widehat{f}(\xi)=\sum_{x \in \mathbf{G}} f(x) \overline{\xi(x)} .
$$

Нам понадобятся несколько известных тождеств, касающихся преобразования Фурье:

$$
\begin{gathered}
\|f\|_{2}^{2}:=\sum_{x \in \mathbf{G}}|f(x)|^{2}=\frac{1}{N} \sum_{\xi \in \widehat{\mathbf{G}}}|\widehat{f}(\xi)|^{2}=\frac{1}{N}\|\widehat{f}\|_{2}^{2} \quad \text { (равенство Парсеваля); } \\
\langle f, g\rangle:=\sum_{x \in \mathbf{G}} f(x) \overline{g(x)}=\frac{1}{N} \sum_{\xi \in \widehat{\mathbf{G}}} \widehat{f}(\xi) \overline{\widehat{g}(\xi)}=\frac{1}{N}\langle\widehat{f}, \widehat{g}\rangle \quad \text { (тождество Планшереля); } \\
\sum_{x \in \mathbf{G}}|(f * g)(x)|^{2}=\sum_{x \in \mathbf{G}}\left|\sum_{y \in \mathbf{G}} f(y) g(x-y)\right|^{2}=\frac{1}{N} \sum_{\xi \in \widehat{\mathbf{G}}}|\widehat{f}(\xi)|^{2}|\widehat{g}(\xi)|^{2} ; \\
f(x)=\frac{1}{N} \sum_{\xi \in \widehat{\mathbf{G}}} \widehat{f}(\xi) \xi(x) \quad(\text { формула обращения); } \\
\widehat{f * g}=\widehat{f} \widehat{g} \quad \text { и }(\widehat{f g})(x)=\frac{1}{N}(\widehat{f} * \widehat{g})(x) .
\end{gathered}
$$




\section{Будем писать для краткости $\sum_{s}$ вместо $\sum_{s \in \mathbf{G}}$ и $\sum_{\xi}$ вместо $\sum_{\xi \in \widehat{\mathbf{G}}}$.}

Приведем два примера групп, которые будут особенно часто использоваться в нашем обзоре.

Пример 1. Пусть $N$ - натуральное число и $\mathbf{G}=\mathbb{Z} / N \mathbb{Z}=\mathbb{Z}_{N}$. Тогда все характеры - это корни из единицы степени $N$, т. е. $e^{2 \pi i r / N}$, где $r=0,1, \ldots, N-1$. Будем писать $e_{N}(x)$ вместо $e^{2 \pi i x / N}$. Тогда преобразование Фурье функции $f$ задается формулой

$$
(\Phi f)(\xi)=\widehat{f}(\xi)=\sum_{x \in \mathbb{Z}_{N}} f(x) e_{N}(-\xi x)
$$

В группе $\mathbb{Z}_{N}$ можно описать структуру множеств Бора (2). Простые рассуждения, связанные с принципом Дирихле, позволяют доказать следующую лемму (см., например, $[2 ; \S 4.4])$.

Лемма 1. Пусть $\mathbf{G}=\mathbb{Z}_{N} u|\Gamma|=d$. Тогда множество Бора $\mathscr{B}(\Gamma, \varepsilon)$ содержит арифметическую прогрессию длины $\varepsilon N^{1 / d}$.

Таким образом, множество Бора можно представлять себе как объединение арифметических прогрессий.

ПримеР 2. Пусть $p$ - простое число, $n$ - натуральное число и $\mathbf{G}=\mathbb{F}_{p}^{n}=$ $(\mathbb{Z} / p \mathbb{Z})^{n}$. Группа $\mathbf{G}$ является векторным пространством со скалярным произведением

$$
x \cdot y=\mathbf{x} \cdot \mathbf{y}=\langle\mathbf{x}, \mathbf{y}\rangle=x_{1} y_{1}+\cdots+x_{n} y_{n} \quad(\bmod p) .
$$

Преобразование Фурье функции $f: \mathbb{F}_{p}^{n} \rightarrow \mathbb{C}$ вычисляется по формуле

$$
\widehat{f}(r)=\sum_{x \in \mathbb{F}_{p}^{n}} f(x) e_{p}(-\langle r, x\rangle)
$$

где $e_{p}(x)=e^{2 \pi i x / p}$. Особенно просто устроено преобразование Фурье в группе $\mathbb{F}_{2}^{n}$. В самом деле, так как $e_{2}(x)=(-1)^{x}$, то для действительной функции $f$ числа $\widehat{f}(r)$ - действительные.

В группах $\mathbb{F}_{p}^{n}$ понятию множества Бора соответствует понятие аффинного подпространства. Пусть $\mathbf{v}_{1}, \ldots, \mathbf{v}_{k}$ - некоторые линейно независимые векторы, и пусть $c_{1}, \ldots, c_{k}-$ произвольные элементы $\mathbb{F}_{p}$. Аффинным подпространством коразмерности $k$ называется множество

$$
P=P_{c_{1}, \ldots, c_{k}}\left(\mathbf{v}_{1}, \ldots, \mathbf{v}_{k}\right)=\left\{\mathbf{x} \in \mathbb{F}_{p}^{n}:\left\langle\mathbf{x}, \mathbf{v}_{1}\right\rangle=c_{1}, \ldots,\left\langle\mathbf{x}, \mathbf{v}_{k}\right\rangle=c_{k}\right\} .
$$

Если все $c_{i}$ равны нулю, то иногда мы вместо $P_{0, \ldots, 0}\left(\mathbf{v}_{1}, \ldots, \mathbf{v}_{k}\right)$ будем писать $P\left(\mathbf{v}_{1}, \ldots, \mathbf{v}_{k}\right)$. Для множества $W=\left(w_{1}, \ldots, w_{|W|}\right)$ пусть $P(W)$ означает $P\left(w_{1}, \ldots, w_{|W|}\right)$. Преобразование Фурье характеристической функции $\chi_{P}$ множества $P=P(W)$ вычисляется чрезвычайно просто. Пусть $L-$ линейное пространство размерности $k$, натянутое на векторы $\mathbf{v}_{1}, \ldots, \mathbf{v}_{k}$, и пусть $\mathbf{r} \in \mathbb{F}_{p}^{n}-$ 
произвольный вектор. Ясно, что $P^{\perp}=L$ и для всякого $r \in L$ имеет место равенство $\widehat{\chi}_{P}(r)=|P|$. Далее, из равенства Парсеваля

$$
\sum_{r \in \mathbb{F}_{p}^{n}}\left|\widehat{\chi}_{P}(r)\right|^{2}=|P| N
$$

вытекает, что $\widehat{\chi}_{P}(r)=0$ для любого $r \notin L$. Иными словами,

$$
\widehat{\chi}_{P}(r)=\chi_{L}(r)|P|,
$$

где $\chi_{L}-$ характеристическая функция множества $L$. Таким образом, $\left|\widehat{\chi}_{P}(r)\right|$ либо нуль, либо равен $|P|$.

Аддитивной энергией двух конечных множеств $A, B \subseteq \mathbf{G}$ (см., например, [2; $\S 2.3])$ называется величина $\mathrm{E}(A, B)$, равная числу решений уравнения

$$
\mathrm{E}(A, B)=\left|\left\{a_{1}+b_{1}=a_{2}+b_{2}: a_{1}, a_{2} \in A, b_{1}, b_{2} \in B\right\}\right| .
$$

Если $A=B$, то мы просто пишем $\mathrm{E}(A)$ вместо $\mathrm{E}(A, A)$. Ясно, что

$$
\mathrm{E}(A, B)=\sum_{x}(A * B)^{2}(x)=\sum_{x}(A \circ B)^{2}(x)=\sum_{x}(A \circ A)(x)(B \circ B)(x)
$$

и, в случае конечной группы $\mathbf{G}$, по формуле (7) имеем

$$
\mathrm{E}(A, B)=\frac{1}{N} \sum_{\xi}|\widehat{A}(\xi)|^{2}|\widehat{B}(\xi)|^{2} .
$$

Аддитивная энергия множеств $A$ и $B$ связана с мощностью их суммы или разности неравенством, получающимся применением неравенства Коши-Буняковского:

$$
|A \pm B| \mathrm{E}(A, B) \geqslant|A|^{2}|B|^{2} .
$$

Пусть теперь $k$ - натуральное число и $A \subseteq \mathbf{G}$ - произвольное конечное множество. Энергиями $\mathrm{E}_{k}(A)$ (или, по-другому, старшими моментами сверток, см. [21]) называются величины

$$
\mathrm{E}_{k}(A)=\left|\left\{a_{1}-a_{1}^{\prime}=a_{2}-a_{2}^{\prime}=\cdots=a_{k}-a_{k}^{\prime}: a_{1}, a_{1}^{\prime}, \ldots, a_{k}, a_{k}^{\prime} \in A\right\}\right| .
$$

Если взять $k=2$, то мы получим обычную аддитивную энергию $\mathrm{E}_{2}(A)=\mathrm{E}(A)$. Можно рассмотреть и граничный случай $k=1$. В этой ситуации $\mathrm{E}_{1}(A)=$ $|A|^{2}$, поскольку соотношение (14) не дает нам никаких ограничений. Таким образом, мощность множества можно рассматривать как некий вырожденный тип энергии. Отметим, что тривиальная верхняя оценка на энергию $\mathrm{E}_{k}(A)-$ это $|A|^{k+1}$.

Чуть более общо, для вектора $s=\left(s_{1}, \ldots, s_{k-1}\right)$ положим $A_{s}^{B}=B \cap\left(A-s_{1}\right) \cap$ $\cdots \cap\left(A-s_{k-1}\right)$. Если $B=A$, то мы пишем $A_{s}$ вместо $A_{s}^{A}$. Тогда

$$
\mathrm{E}_{k}(A)=\sum_{x \in \mathbf{G}}(A \circ A)^{k}(x)=\sum_{s_{1}, \ldots, s_{k-1} \in \mathbf{G}}\left|A_{s}\right|^{2}
$$


и формула

$$
\mathrm{E}_{k}(A, B)=\sum_{x \in \mathbf{G}}(A \circ A)(x)(B \circ B)^{k-1}(x)=\sum_{s_{1}, \ldots, s_{k-1} \in \mathbf{G}}\left|B_{s}^{A}\right|^{2}
$$

задает совместную $k$-ю энергию множеств $A$ и $B$. Первые равенства в $(15),(16)$ можно рассматривать как определения величин $\mathrm{E}_{k}(A), \mathrm{E}_{k}(A, B)$ для нецелых $k$, $k \geqslant 1$. Для $P \subseteq A-A$ мы пишем $\mathrm{E}_{k}^{P}(A):=\sum_{x \in P}(A \circ A)^{k}(x)$.

Очевидно, что

$$
\begin{aligned}
\mathrm{E}_{k+1}(A, B) & =\sum_{x}(A \circ A)(x)(B \circ B)^{k}(x) \\
& =\sum_{x_{1}, \ldots, x_{k}}\left(\sum_{y} A(y) B\left(y+x_{1}\right) \cdots B\left(y+x_{k}\right)\right)^{2}=\mathrm{E}\left(\Delta_{k}(A), B^{k}\right),
\end{aligned}
$$

где $\Delta_{k}(A)$ - диагональ:

$$
\Delta(A)=\Delta_{k}(A):=\left\{(a, a, \ldots, a) \in A^{k}\right\} .
$$

Для $x \in \mathbf{G}$ положим $\Delta(x)=\Delta_{k}(x)=(x, \ldots, x) \in \mathbf{G}^{k}$.

Также нам понадобятся "дуальные" к $\mathrm{E}_{k}(A)$ величины, хорошо известные в аналитической теории чисел [30], [31]:

$$
\begin{aligned}
\mathrm{T}_{k}(A) & :=\left|\left\{a_{1}+\cdots+a_{k}=a_{1}^{\prime}+\cdots+a_{k}^{\prime}: a_{1}, \ldots, a_{k}, a_{1}^{\prime}, \ldots, a_{k}^{\prime} \in A\right\}\right| \\
& =\frac{1}{N} \sum_{\xi}|\widehat{A}(\xi)|^{2 k} .
\end{aligned}
$$

По поводу дуальности $\mathrm{E}_{k}$ и $\mathrm{T}_{k}$ см. [21]-[23]. Например, в [22; замечание 6.6] было доказано, что

$$
\left(\frac{\mathrm{E}_{3 / 2}(A)}{|A|}\right)^{2 k} \leqslant \mathrm{E}_{k}(A) \mathrm{T}_{k}(A)
$$

если число $k$ - четное. Более того, из формул (4)-(8) вытекает, что $\mathrm{T}_{k}\left(|\widehat{A}|^{2}\right)=$ $N^{2 k-1} \mathrm{E}_{2 k}(A)$ и $\sum_{x}(\widehat{\widehat{A}} \circ \widehat{A})^{k}(x)(\widehat{A} \circ \widehat{\widehat{A}})^{k}(x)=N^{2 k+1} \mathrm{~T}_{k}(A)$.

Сделаем еще несколько замечаний об обозначениях. Если $S \subseteq \mathbf{G}$ - некоторое множество, то $S(x)$ обозначает его характеристическую функцию. Иными словами, $S(x)=1$, если $x \in S$, и $S(x)=0$ в противном случае. Числа $\widehat{S}$ называют коэффициентами Фурье множества $S$. Символ $|S|$ используется для мощности множества $S$. Если $A, B \subseteq \mathbf{G}-$ произвольные множества и $\mu_{\mathbf{G}}(A)>0$, то $\mu_{A}(B):=\mu_{\mathbf{G}}(B) / \mu_{\mathbf{G}}(A)$. Мы также используем обозначение $\mu_{A}(x)$ для функции $A(x) /|A|$. Запись $\log$ означает логарифм по основанию два. Знаки « и суть обычные символы Виноградова, и если оценки в неравенствах зависят от некоторого параметра $M$ полиномиально, то мы пишем $\ll_{M}$, $\gg_{M}$. Если для двух величин $a, b$ выполнены неравенства $a \ll_{M} b, b \ll_{M} a$, то мы записываем этот факт следующим образом: $a \sim_{M} b$. В частности, символ $a \sim b$ означает, что $a \ll b$ и одновременно $b \ll a$. Если $n-$ натуральное число, то будем писать $[n]$ для отрезка натурального ряда $\{1,2, \ldots, n\}$. Через $\mathscr{P}$ обозначим множество простых чисел. Мы используем обозначение $e(x)$ для функции $e(x)=e^{2 \pi i x}$. Наконец, $\mathbb{Z}_{N}^{*}=\mathbb{Z}_{N} \backslash\{0\}$. 


\section{3. Примеры семейств аддитивных множеств}

В этом разделе мы разберем несколько модельных примеров аддитивных множеств, которые понадобятся нам впоследствии. Часто рассматривается лишь простейший случай группы $\mathbf{G}=\mathbb{F}_{2}^{n}$, поскольку мы считаем нашей основной задачей наиболее наглядно продемонстрировать имеющиеся различия между комбинаторными свойствами различных семейств множеств, а не охватить все их многообразие. Важность групп $\mathbb{F}_{p}^{n}$ обсуждается в прекрасном обзоре [3].

$\mathrm{C}$ точки зрения аддитивной комбинаторики исторически первым примером семейства аддитивных множеств были случайные множества, естественным образом возникающие в рамках вероятностного метода [32].

Пример 3. Пусть группа $\mathbf{G}$ - конечная, $N=|\mathbf{G}|, \delta \in(0,1]$ - произвольное число и $A$ - "случайное" подмножество $\mathbf{G}$ такое, что каждый элемент множества $A$ принадлежит ему с вероятностью $\delta$. Говоря более точно, пусть $\Omega-$ пространство последовательностей длины $N$, состоящих из нулей и единиц, а $\mathscr{F}$ - сигма-алгебра всех подмножеств $\Omega$. Если последовательность $\omega \in \Omega$ имеет $k$ единиц и $N-k$ нулей, то припишем этой последовательности вероятность $\delta^{k}(1-\delta)^{N-k}$. Мы задали вероятностное пространство $(\Omega, \mathscr{F}, \mathbf{P})$. Каждой точке $\omega=\left(\omega_{1}, \omega_{2}, \ldots, \omega_{N}\right)$ множества $\Omega$ поставим в соответствие множество $A(\omega)=\left\{i: \omega_{i}=1\right\}$. Случайные множества - это множества $A(\omega)$ для "типичных" последовательностей $\omega$. Очень многие характеристики случайных множеств могут быть легко посчитаны. Например, несложно показать, что математическое ожидание мощности множества $A(\omega)$ равно $\delta N$. Исходя из этого факта говорят, что мощность случайного множества в заданной выше вероятностной модели равна $\delta N$. Аналогично можно найти математическое ожидание мощности множества $|A+A|$. Оно оказывается по порядку равным величине $N$, если $\delta>N^{-1 / 2}$. Таким образом, сумма случайного множества с собой заполняет почти всю группу. Легко показать, что аддитивная энергия $\mathrm{E}(A)$ случайного множества с большой вероятностью (точнее, с вероятностью $1-o(1), N \rightarrow \infty)$ равна $\delta^{4} N^{3}$. Это легко увидеть из того, что в уравнении для аддитивной энергии $x+y=z+w, x, y, z, w \in A$, каждый из четырех элементов $x, y, z, w$ выбирается независимо с вероятностью $\delta$, кроме тех редких случаев, когда некоторые элементы оказываются совпадающими. Далее, пользуясь оценками больших уклонений для последовательности независимых одинаково распределенных случайных величин, можно также доказать, что все ненулевые коэффициенты Фурье случайного множества не превосходят $N^{1 / 2+\varepsilon}$, где $\varepsilon>0$ - произвольное (более подробно см., например, [32]). Более того, случайные множества представляют собой пример равномерных множеств, т.е. множеств с малыми нормами Гауэрса (см. [4], [33] и раздел 8). Наконец, можно показать, что в некотором смысле "почти все" подмножества $\mathbf{G}$ мощности $\delta N$ являются случайными.

Итак, случайные множества - это первый, достаточно простой пример аддитивных множеств. С аддитивно-комбинаторной точки зрения аналитические методы (см., например, [31], [34], [35]), такие как оценки тригонометрических 
сумм, т.е. коэффициентов Фурье характеристических функций, суть утверждения о том, что данное конкретное множество является в некотором смысле близким к случайному (см., например, статью [9] или обсуждение в 4 обзоpa [33]).

Противоположностью случайным множествам являются структурированнъе объекты. Мы приведем список семейств множеств, которые считаются структурированными в аддитивной комбинаторике.

ПримеР 4. Пусть $\mathbf{G}=\mathbb{Z}$ и $a \in \mathbb{Z}, d \in \mathbb{Z}, d \neq 0,-$ некоторые числа. Тогда множество $A=\{a, a+d, a+2 d, \ldots, a+(k-1) d\}$ - это обыкновенная арифметическая прогрессия с начальным элементом $a$ и разностью $d$. Такие множества задаются минимальным числом параметров, а именно двумя числами $a$ и $d$, и поэтому они считаются чрезвычайно структурированными. Легко вычислить все характеристики арифметической прогрессии $A$. Например, $|A+A|=2|A|-1$ и $\mathrm{E}(A)=\left(2|A|^{3}+|A|\right) / 3$. Сумма $Q=P_{1}+\cdots+P_{k}$ арифметических прогрессий $P_{1}, \ldots, P_{k}$ называется обобщенной арифметической прогрессией. При этом количество арифметических прогрессий $k$ называется размерностью обобщенной арифметической прогрессии. Вообще говоря, мощность $Q$ может быть меньше величины $\left|P_{1}\right| \cdots\left|P_{d}\right|$, но если $|Q|=\left|P_{1}\right| \cdots\left|P_{d}\right|$, то такая обобщенная арифметическая прогрессия называется точной. В ситуации, когда число $k$ достаточно мало, множество $Q$ также считается структурированным и его характеристики несложно найти. Например, легко видеть, что $|Q+Q| \leqslant 2^{k}|Q|$. Наконец, если удалить из арифметической прогрессии или из обобщенной арифметической прогрессии небольшое число элементов, то это не слишком изменит аддитивную структуру множества. Например, если $A \subseteq Q,|A| \geqslant \beta|Q|$, то, очевидно,

$$
|A+A| \leqslant|Q+Q| \leqslant 2^{k}|Q| \leqslant \beta^{-1} \cdot 2^{k}|A|
$$

и, значит, сумма множества $A$ с собой сравнима по мощности с $|Q+Q|$. В аддитивной комбинаторике "плотные" подмножества обобщенных арифметических прогрессий "малой” размерности считаются структурированными. Точный смысл слов "плотный" и "малый" обсуждается в разделе 4. Еще более сложными объектами, имеющими структуру, являются уже упоминавшиеся множества Бора (2).

Рассмотрим теперь группу $\mathbb{F}_{p}^{n}, p$ - простое. В таких группах обобщенные арифметические прогрессии и множества Бора совпадают между собой (см. раздел 2), и, как мы уже знаем, все они соответствуют семейству аффинных подпространств. Это объясняет, в частности, почему удобно рассматривать в качестве обертывающих групп $\mathbf{G}$ именно группы $\mathbb{F}_{p}^{n}$. Наконец, если мы имеем любое подпространство $H$, то независимо от его размерности $|H+H|=|H|$ и $\mathrm{E}(H)=|H|^{3}$.

Обычно для изучения случайных множеств используют методы теории вероятностей или же аналитический подход [34]. Для описания множеств из примера 4 (а также приводимых ниже примеров 5-7) применяют совершенно 
другие методы. Собственно, множества из примера 4 вплоть до самого последнего времени являлись основными объектами изучения аддитивной комбинаторики. Совсем недавно проблематика данной науки существенно расширилась, в том смысле, что теперь все чаще и чаще аддитивной комбинаторике приходится иметь дело с множествами, не подпадающими под определения из примеров 3,4 .

Приведем лишь два таких примера. Первый из них представляет собой смесь случайного и структурированного случаев. Мы будем называть такие объекты множествами вида $H \dot{+} \Lambda$.

ПримеР 5. Пусть $\mathbf{G}=\mathbb{F}_{2}^{n}, K$ - натуральное число, $K<n$. Рассмотрим первые $K$ векторов стандартного базиса $e_{1}=(1,0, \ldots, 0), e_{2}=(0,1,0, \ldots, 0), \ldots$, $e_{K}=(0, \ldots, 0,1,0, \ldots, 0)$. Положим $\Lambda=\left\{e_{1}, \ldots, e_{K}\right\}$, и пусть $H$ - произвольное подпространство в пространстве, натянутом на базисные векторы $e_{K+1}, \ldots, e_{n}$. Наконец, наше множество $A$ - это (прямая) сумма $H \dot{+} \Lambda$. Ясно, что $|A|=|H||\Lambda|$ и $A+A=H \dot{+} \Lambda \dot{+} \Lambda$. Вместо базиса $\Lambda$ можно также брать и произвольное случайное подмножество подпространства, натянутого на векторы $\left\{e_{1}, \ldots, e_{K}\right\}$, структура суммы $A+A$ от этого изменится не слишком сильно.

Важность данного семейства множеств в том, что они совмещают в себе одновременно структурные и случайные свойства. Действительно, с одной стороны, множество $A$ состоит из трансляций подпространства $H$, а с другой стороны, оно является случайным, например, в смысле анализа Фурье (более подробно см. раздел 8), поскольку $\widehat{A}=\widehat{H} \widehat{\Lambda}$ и коэффициенты Фурье множества $\Lambda$ малы. Неудивительно, что из-за совмещения этих двух свойств множества $A$ такого вида часто оказываются контрпримерами в различных аддитивно-комбинаторных задачах.

Следующий важный пример семейства аддитивных множеств, связанный с тематикой раздела 4, был любезно сообщен автору Т. Шоеном. Это семейство называется картошками Шоена. Несложно видеть, что оно обобщает множества $H \dot{+} \Lambda$.

ПримеР 6. Пусть $1 \leqslant M \leqslant K$ - действительные числа. Рассмотрим множество вида $H \dot{+} \Lambda$, где $|\Lambda|=M$. Для каждого $\lambda \in \Lambda$ пусть $B_{\lambda}$ - случайные подмножества $H+\lambda$, имеющие одинаковую мощность (она будет равна $|A| / M$, размер $A$ - это еще один параметр). Положим $A=\bigsqcup_{\lambda \in \Lambda} B_{\lambda}$. Наконец, пусть подпространство $H$ имеет мощность $|H|=K|A| / M^{2}=\delta^{-1}\left|B_{\lambda}\right|$, где $\lambda \in \Lambda$, $\delta=M / K$. Ясно, что в случае $M=K$ мы получаем рассмотренную ранее ситуацию $A=H \dot{+} \Lambda$. Поскольку множества $B_{\lambda}$ - случайные, легко видеть, что $\mathrm{E}(A)=\sum_{\lambda_{1}, \lambda_{2} \in \Lambda} \mathrm{E}\left(B_{\lambda_{1}}, B_{\lambda_{2}}\right) \sim \delta^{4}|H|^{3} M^{2}=|A|^{3} / K$ при условии, что параметр $K$ достаточно мал по сравнению с параметром $|A|$. Аналогично,

$$
\begin{aligned}
\mathrm{E}_{3}(A) & =\sum_{\lambda_{1}, \lambda_{2}, \lambda_{3} \in \Lambda} \sum_{x}\left(B_{\lambda_{1}} \circ B_{\lambda_{1}}\right)(x)\left(B_{\lambda_{2}} \circ B_{\lambda_{2}}\right)(x)\left(B_{\lambda_{3}} \circ B_{\lambda_{3}}\right)(x) \\
& \sim \delta^{6}|H|^{4} M^{3}=\frac{M|A|^{4}}{K^{2}} .
\end{aligned}
$$


Далее, $|A+A| \sim M^{2}|H|=K|A|$, если, опять же, параметр $K$ достаточно мал по сравнению с параметром $|A|$.

Найдем теперь аддитивные характеристики подмножеств $A_{s}=A \cap(A-s)$. Эти множества делятся на два класса, оба вносят одинаковый по порядку вклад в аддитивную энергию нашего множества $A$. Такая ситуация будет и в общем случае произвольного аддитивного множества (см. работы [19], [20], а также [22]).

1) "Малые" $A_{s}, s \in H \dot{+} \Lambda \dot{+} \Lambda$, т. е. $s \notin H$. В этом случае $\left|A_{s}\right| \sim \delta^{2}|H|=|A| / K$, поскольку множества $B_{\lambda}$ выбираются случайно. Далее, удобно найти сначала характеристики множеств $B_{\lambda}$. Поскольку характеристики при разных $\lambda$ одни и те же, для обозначения произвольного множества $B_{\lambda}$ мы будем использовать букву В. Итак,

$$
\begin{gathered}
|B|=\frac{|A|}{M}, \quad|B+B| \sim|H|=\frac{K|B|}{M}, \quad|A+B| \sim M|H|=\frac{K|A|}{M}, \\
\mathrm{E}(B) \sim \delta^{4}|H|^{3} \sim \frac{|A|^{3}}{M^{2} K} \sim \frac{M|B|^{3}}{K}, \quad \mathrm{E}(A, B)=M \mathrm{E}(B) \sim \frac{|A|^{3}}{M K} \sim \frac{M|B|^{2}|A|}{K} .
\end{gathered}
$$

Отсюда следует, что

$$
\left|A_{s}+A_{s}\right| \sim|H|=\frac{K|A|}{M^{2}} \quad \text { и }\left|A+A_{s}\right| \sim|H| M=\frac{K|A|}{M} .
$$

Наконец,

$$
\begin{gathered}
\mathrm{E}\left(A_{s}\right) \sim \delta^{8}|H|^{3} \sim \frac{M^{2}|A|^{3}}{K^{5}} \sim \frac{M^{2}\left|A_{s}\right|^{3}}{K^{2}}, \\
\mathrm{E}\left(A, A_{s}\right)=M \mathrm{E}\left(A_{s}\right) \sim \frac{M^{3}|A|^{3}}{K^{5}} \sim \frac{M^{3}}{K^{3}}\left|A_{s}\right|^{2}|A| .
\end{gathered}
$$

2) "Большие" $A_{s}, s \in H$, которые, как легко видеть, в $M$ раз больше, чем "малые" $A_{s}$. Иными словами, $\left|A_{s}\right| \sim M|A| / K$. Для этих множеств выполнены соотношения $\left|A_{s}+A_{s}\right| \sim\left|A+A_{s}\right| \sim|H| M^{2}=K|A|$. Пользуясь предыдущими вычислениями, получаем

$$
\begin{gathered}
\mathrm{E}\left(A_{s}\right) \sim M^{2} \delta^{8}|H|^{3} \sim \frac{M^{4}|A|^{3}}{K^{5}} \sim \frac{M\left|A_{s}\right|^{3}}{K^{2}}, \\
\mathrm{E}\left(A, A_{s}\right) \sim M^{2} \delta^{6}|H|^{3} \sim \frac{M^{2}|A|^{3}}{K^{3}} \sim \frac{\left|A_{s}\right|^{2}|A|}{K} .
\end{gathered}
$$

Наконец, последнее семейство, которое можно назвать семейством самодуальных множеств (см. [22]), также часто является контрпримером по многих задачах. Скажем, конструкции самодуальных множеств показывают, что суммы $A+A$ (или, более общо, множества так называемых популярных разностей, см. $[2 ; \S 2.6]$ или $[9 ;$ доказательство предложения 7.3]), вообще говоря, не являются структурированными множествами в смысле примера 4.

Пример 7. Пусть $k$ - натуральное число и $S_{1}, \ldots, S_{k} \subseteq[n]-$ произвольные непересекающиеся множества одинакового размера. Пусть также $H_{1}, \ldots$, $H_{k} \subseteq \mathbb{F}_{2}^{n}$ - подпространства такие, что каждое $H_{j}$ натянуто на векторы $e_{i_{j}}, i_{j} \in S_{j}$. Подпространства $H_{j}$ имеют одинаковую мощность, которую мы 
обозначим буквой $h$. Будем считать, что параметр $k$ достаточно мал по отношению к $h$. Наконец, наше самодуальное множество $A$ - это (дизъюнктное) объединение всех подпространств $H_{1}, \ldots, H_{k}$. Ясно, что

$$
A+A=A \cup\left(\bigcup_{i \neq j}\left(H_{i}+H_{j}\right)\right)
$$

и, следовательно, $|A+A| \gg|A|^{2}$. Иными словами, множество $A+A$ для самодуальных множеств очень большое. С другой стороны,

$$
\mathrm{E}(A)=\sum_{j=1}^{k} \mathrm{E}\left(H_{j}\right)+\sum_{i \neq j} \mathrm{E}\left(H_{i}, H_{j}\right)=k h^{3}+\frac{k(k-1)}{2} h^{2} \sim \sum_{j=1}^{k} \mathrm{E}\left(H_{j}\right)=\frac{|A|^{3}}{k^{2}},
$$

поскольку, по нашей договоренности, параметр $k$ достаточно мал по отношению к $h$. Таким образом, энергия множества $A$ сосредоточена в подпространствах $H_{j}$ и смешанные энергии не вносят существенного вклада в $\mathrm{E}(A)$.

Аналогом самодуальных множеств в целых числах являются объединения арифметических прогрессий $H_{1}, \ldots, H_{k}$ одинаковой длины $h$, но с "аддитивно независимыми" разностями, например, $\left\{1,(3 h),(3 h)^{2}, \ldots,(3 h)^{k-1}\right\}$. При таком выборе разностей наши расчеты для мощности $A+A$, а также вычисления в формуле (18) останутся в силе. Тем не менее мы предпочитаем рассматривать самодуальные множества в $\mathbb{F}_{2}^{n}$, поскольку, во-первых, их устройство несколько проще и, во-вторых, подпространства $H_{1}, \ldots, H_{k}$ обладают дополнительным полезным свойством "обобщенной аддитивной независимости" $\left|H_{i_{1}}+\cdots+H_{i_{l}}\right|=\left|H_{i_{1}}\right| \cdots\left|H_{i_{l}}\right|$, если индексы $i_{1}, \ldots, i_{l}$ различны. Смысл этого равенства, конечно, состоит в том, что между элементами $H_{1}, \ldots, H_{k}$ нет никаких нетривиальных аддитивных соотношений.

\section{4. Строение множеств с малым удвоением}

В этом разделе мы рассмотрим классический вопрос аддитивной комбинаторики (см. [1], [2], [7]), а также затронем имеющиеся здесь многочисленные приложения. Этот вопрос формулируется так: какова структура множества $A$, если известно, что величины $|A|$ и $|A+A|$ находятся в критическом соотношении, иными словами, если $|A+A|$ не слишком велико по отношению к $|A|$ ? Мы четко сформулируем данную проблему чуть ниже, а пока отметим, что совсем недавно в этой задаче наметился существенный прогресс, который связан, прежде всего, с результатами Шоена [36], Сандерса [37] и Конягина (см. [27]).

Константой удвоения множества $A \subseteq \mathbf{G}$ называется число

$$
\mathrm{D}=\mathrm{D}[A]:=\frac{|A+A|}{|A|} .
$$

Это важная характеристика множества, и мы видели в разделе 3, что различные аддитивные семейства могут существенно отличаться величиной D. Так, для случайного множества $A \subseteq \mathbf{G}$ имеем $|A+A| \gg \min \left\{|\mathbf{G}|,|A|^{2}\right\}$, т. е. константа удвоения случайного множества - большая. В этом разделе мы рассмотрим 
противоположную ситуацию и разберем случай, когда число D “мало”. Чуть более формально это означает, что нас интересует тот режим, когда мощность множества $A$ растет, а константа D фиксирована или же изменяется с изменением мощности $A$ очень медленно. Такие множества называют множествами с малым удвоением. Можно ожидать, что структура подобных множеств весьма неслучайна.

Ясно, что всегда $\mathrm{D} \geqslant 1$, и легко показать, что $\mathrm{D}[A]=1$ тогда и только тогда, когда $A$ является смежным классом некоторой подгруппы G. Таким образом, равенство $\mathrm{D}[A]=1$ выделяет смежные классы среди всего многообразия аддитивных множеств. Что будет, если рассмотреть бо́льшие константы удвоения? Например, если множество $A$ является подмножеством некоторого смежного класса $\Gamma$ и $|A| \geqslant|\Gamma| / K, K \geqslant 1$, то ясно, что $\mathrm{D}[A] \leqslant K$, поскольку $|A+A| \leqslant|\Gamma+\Gamma|=|\Gamma| \leqslant K|A|$. Замечательно, что при очень маленьких $\mathrm{D}[A]$ других примеров множеств с малым удвоением нет (см., например, $[2 ; \S 5.1])$.

Теорема 1. Пусть $A \subseteq \mathbf{G}$ - произвольное множество. Предположим, что $\mathrm{D}[A]<3 / 2$. Тогда найдутся подгруппа $\Gamma,|\Gamma| \leqslant \mathrm{D}[A]|A|$, и элемент $x \in \mathbf{G}$ maкuе, что $A \subseteq \Gamma+x$.

Константа $3 / 2$ в теореме 1 является точной. Действительно, пусть $\mathbf{G}=\mathbb{F}_{4}^{n}$ и множество $A$ равно дизъюнктному объединению некоторого подпространства $H$ и его сдвига $H+x$, где порядок элемента $x$ равен 4 . Тогда $\mathrm{D}[A]=$ $3 / 2$ и любое подпространство $\Gamma$, содержащее $A$, имеет мощность не меньше, чем $2|A|$. Таким образом, если мы хотим обобщить теорему 1 на бо́льшие константы удвоения, то ясно, что для описания соответствующих множеств $A$ нам потребуется использовать не менее двух смежных классов подгруппы Г. В следующей теореме рассматривается эта более сложная ситуация [27], [38].

Tеорема 2. Пусть $A \subseteq \mathbf{G}-$ произвольное множество и $\varepsilon \in(0,1)-$ действительное число. Предположим, что $\mathrm{D}[A]<2-\varepsilon$. Тогда найдутся подгруппа $\Gamma,|\Gamma| \leqslant \mathrm{D}[A]|A|$, и элементы $x_{1}, \ldots, x_{k} \in \mathbf{G}, k \ll_{\varepsilon} 1$, такие, что

$$
A \subseteq \bigsqcup_{j=1}^{k}\left(\Gamma+x_{j}\right) .
$$

В отличие от теоремы 1 , теорема 2 не является критерием в том смысле, что множества, удовлетворяющие (19), не обязаны иметь константу удвоения меньшую, чем 2 (более точное описание таких множеств дано в работе [38]). С другой стороны, для констант удвоения больше двух дело обстоит еще хуже, поскольку даже покрытий вида (19) для них недостаточно. Действительно, в примере 4 раздела 3 мы видели, что константа удвоения любой арифметической прогрессии в $\mathbb{Z}$ или $\mathbb{R}$ равна $2-1 /|A|<2$. Далее, единственная конечная подгруппа $\mathbb{Z}$ - это $\{0\}$. Значит, если бы для $\mathrm{D}>2$ был выполнен какой-то аналог (19), то любое такое множество $A$ покрывалось бы $O(1)$ элементами, что, конечно, невозможно. Этот пример показывает, что для описания множеств c $\mathrm{D}>2$, помимо смежных классов, необходимо рассматривать и арифметические прогрессии. В примере 4 мы изучали также и обобщенные арифметические прогрессии, т. е. суммы арифметических прогрессий, и указали, что если 
взять сумму небольшого числа таких прогрессий, то константа удвоения полученного множества будет маленькой. Оказывается, такой подход уже дает критерий того, что множество $A$ имеет малую константу удвоения, причем $\mathrm{D}[A]$ может здесь быть гораздо больше, чем 2, и даже зависеть (впрочем, достаточно слабо) от мощности $A$. Фрейман доказал в [7] этот критерий для случая $\mathbf{G}=\mathbb{Z}$ (теорема 3 ниже), а общая теорема (теорема 4 ниже) принадлежит Грину и Руже [39] (см. также [2; §5.6]).

ТеОРема 3. Пусть $A \subseteq \mathbb{Z}$ - произвольное множество с константой удвоения $\mathrm{D}=\mathrm{D}[A]$. Тогда найдутся точная обобщенная арифметическая прогрессия $Q,|Q| \leqslant \exp \{O(f(\mathrm{D}))\}|A|$, размерности $f(\mathrm{D})$ и множество $X \subseteq \mathbb{Z}$, $|X| \leqslant \exp \{O(f(\mathrm{D}))\}$, maкue, чmo

$$
A \subseteq Q+X
$$

Самое главное в теореме 3 - это, конечно, то, что функция $f(\mathrm{D})$ существует и зависит только от константы удвоения, но не от других характеристик множества $A$. В теореме Фреймана в очередной раз проявилась замечательная особенность аддитивной комбинаторики: если константа удвоения достаточно мала, а это означает в нашей терминологии, что величины $|A|$ и $|A+A|$ находятся в критическом соотношении, то такая ситуация возможна, только если множество $A$ устроено весьма специфическим образом.

Чтобы сформулировать аналог теоремы Фреймана для произвольной абелевой группы, нам понадобится одно дополнительное понятие, вбирающее в себя покрывающие множества из теорем 1,2 и 3. Обобщенной арифметической прогрессией $M$ в группе $\mathbf{G}$ называется прямая сумма $\Gamma+Q$ некоторой подгруппы $\Gamma \subseteq \mathbf{G}$ и (обычной) обобщенной арифметической прогрессии $Q$. Размерность $M$, по определению, равна размерности $Q$. Теперь мы можем сформулировать теорему Грина-Ружи.

Теорема 4. Пусть $A \subseteq \mathbf{G}$ - произвольное множество с константой удвоения $\mathrm{D}=\mathrm{D}[A]$. Тогда найдутся точная обобщенная арифметическая прогрессия $M$ в группе $\mathbf{G},|M| \leqslant \exp \{O(f(\mathrm{D}))\}|A|$, размерности $f(\mathrm{D})$ и множество $X \subseteq \mathbf{G},|X| \leqslant \exp \{O(f(\mathrm{D}))\}$, такие, что

$$
A \subseteq M+X
$$

То, что в данной формулировке теорема 4 является критерием для множества быть множеством с малым удвоением, увидеть достаточно легко. Действительно, если множество $A$ удовлетворяет (21), то

$$
|A+A| \leqslant|M+X+M+X| \leqslant|X|^{2}|M+M| \leqslant \exp \{O(f(\mathrm{D}))\}|\Gamma+Q+\Gamma+Q|
$$

$$
\begin{aligned}
& \leqslant \exp \{O(f(\mathrm{D}))\}|\Gamma||Q+Q| \leqslant \exp \{O(f(\mathrm{D}))\} \cdot 2^{f(\mathrm{D})}|\Gamma||Q| \\
& \leqslant \exp \{O(f(\mathrm{D}))\}|M| \\
& \leqslant \exp \{O(f(\mathrm{D}))\}|A|
\end{aligned}
$$

и, следовательно, множество $A$ является множеством с константой удвоения $\exp \{O(f(\mathrm{D}))\}$ совершенно автоматически. 
Легко видеть, что обобщенная арифметическая прогрессия $Q=P_{1}+\cdots+P_{k}$, $P_{j}=\left\{a_{j}, a_{j}+d_{j}, a_{j}+2 d_{j}, \ldots, a_{j}+\left(s_{j}-1\right) d_{j}\right\}, s_{j}=\left|P_{j}\right|, j \in[k]$, является образом $k$-мерного параллелепипеда

$$
\left[a_{1}, a_{1}+d_{1}, \ldots, a_{1}+\left(s_{1}-1\right) d_{1}\right] \times \cdots \times\left[a_{k}, a_{k}+d_{k}, \ldots, a_{k}+\left(s_{k}-1\right) d_{k}\right] \subseteq \mathbb{Z}^{k}
$$

при проекции $\varphi: \mathbb{Z}^{k} \rightarrow \mathbb{Z}, \varphi\left(x_{1}, \ldots, x_{k}\right)=x_{1}+\cdots+x_{k}$. Поэтому вместо обобщенных арифметических прогрессий можно рассматривать выпуклые прогресcuи, т. е. множества $Q$, которые являются образами произвольных гомоморфизмов $\phi, Q=\phi\left(T \cap \mathbb{Z}^{k}\right)$, где $T$ - некоторое выпуклое центрально-симметричное тело в $\mathbb{R}^{k}$. Ясно, что $\mathrm{D}[T]:=\operatorname{Vol}(T+T) / \operatorname{Vol}(T)=2^{k}=\exp \{O(k)\}$, но также можно показать, что и константа удвоения множества $\phi\left(T \cap \mathbb{Z}^{k}\right)$ есть $\exp \{O(k)\}$ (см., например, [27]). Поэтому вычисления в строчках (22a)-(22c) останутся справедливыми и для этого более общего класса множеств.

В последнее время усилился интерес к обобщениям теоремы Фреймана-Грина-Ружи в неабелевых группах и, вообще, к неабелевым аддитивно-комбинаторным постановкам (см. статьи [40]-[44] и обзор [5]). Заметим, например, что из работ [43], [44] вытекает количественное уточнение известной теоремы Громова о виртуально нильпотентных группах [45].

Итак, мы видели, что с качественной стороны теорема 4 является критерием для множества быть множеством с малым удвоением. С количественной же точки зрения все, разумеется, зависит от поведения функции $f(\mathrm{D})$. Скажем, формула (22) позволяет утверждать только, что константа удвоения множества $A$ есть $\exp \{O(f(\mathrm{D}))\}$, хотя мы знаем, что по условию она равна D. Если разрешить полиномиальные потери, то правильный количественный результат здесь был бы, если $f(\mathrm{D})=\log \mathrm{D}$. Это составляет предмет гипотезы, которую сейчас называют полиномиальной гипотезой Фреймана-Ружи (см., например, [46]), хотя ее выдвинула Каталина Мартон (см. [47]).

ГиПотезА 1. Пусть $A \subseteq \mathbf{G}$ - произвольное множество с константой удвоения $\mathrm{D}=\mathrm{D}[A]$. Тогда найдется точная выпуклая прогрессия $M$ такая, что утверждение теоремы 4 выполнено с $f(\mathrm{D})=\log \mathrm{D}$.

Чрезвычайно просто сформулировать полиномиальную гипотезу Фреймана-Ружи в группе $\mathbb{F}_{2}^{n}$.

ГипотезА 2. Пусть $A \subseteq \mathbb{F}_{2}^{n}$ - произвольное множество с константой удвоения $\mathrm{D}=\mathrm{D}[A]$. Тогда найдется подпространство $H$ такое, что $|A \cap H| \geqslant$ $|A| / \mathrm{D}^{C}$ и линейная оболочка множества $A \cap H$ имеет размер не более $\mathrm{D}^{C}|A|$. Здесь $C>0$ - некоторая абсолютная константа.

Гипотезу 2 можно проитерировать и показать, что множество $A$ состоит из кусочков $A_{j} \subseteq H_{j}$, где $H_{j}$ - подпространства $\mathbb{F}_{2}^{n},\left|H_{j}\right| \ll_{\mathrm{D}}\left|A_{j}\right|$ (см. [46]). Более того, в отличие от самодуальных множеств, из-за условия малого удвоения подпространства $H_{j}$ не могут быть аддитивно независимыми и, значит, структура множества $A$ более похожа на "картошки Шоена" (кроме, разумеется, случайного выбора множеств $A_{j}$ ).

Известно, что гипотеза 2 эквивалентна следующему утверждению о функциях [46]. 
ГиПотезА 3. Пусть $K \geqslant 1$ - действительное число и $f: \mathbb{F}_{2}^{n} \rightarrow \mathbb{F}_{2}^{\infty}$ - произвольная функиия, имеющая не менее $2^{3 n} / K$ аддитивных четверок, т.е. наборов $(x, y, z, w) \in\left(\mathbb{F}_{2}^{n}\right)^{4}$ таких, что $x+y=z+w$ и одновременно $f(x)+f(y)=$ $f(z)+f(w)$. Тогда найдется афбинная функция $g: \mathbb{F}_{2}^{n} \rightarrow \mathbb{F}_{2}^{\infty}$ такая, что $f(x)=g(x)$ для не менее чем $2^{n} / K^{C}$ значений $x \in \mathbb{F}_{2}^{n}$. Здесъ $C>0$-некоторая абсолютная константа.

В настоящее время полиномиальная гипотеза еще не доказана, хотя в последнее время в этой области был достигнут существенный прогресс. Сам Фрейман [7] установил лишь существование функции $f(\mathrm{D})$. Первые количественные, хотя и достаточно слабые, оценки были получены затем в [48]. Ружа [49] и затем Чанг [26] получили экспоненциальную и полиномиальную оценку на $f(\mathrm{D})$ соответственно (см. также [50]-[53]). Первую не полиномиальную оценку вида $f(\mathrm{D})=O\{\exp (O(\sqrt{\log \mathrm{D}}))\}$ нашел Шоен в [36]. Затем, используя новую замечательную технику Крута-Сисаска о почти периодичности сверток (см. [24], [25] и раздел 5), а также ряд других идей, Сандерс [37] доказал, что $f(\mathrm{D})=O\left(\log ^{4+o(1)} \mathrm{D}\right)$. Наконец, Конягин [27] получил наилучшую на сегодняшний день оценку для функции $f(\mathrm{D})$, а именно

$$
f(\mathrm{D})=O\left(\log ^{3+o(1)} \mathrm{D}\right) \text {. }
$$

Таким образом, от полиномиальной гипотезы Фреймана-Ружи результат Конягина отличается только константой $3+o(1)$, которая стоит в степени логарифма.

Полиномиальная гипотеза, если бы она была доказана, имела бы массу важнейших приложений. Часть из этих приложений к задачам о суммах произведений собрана в специально посвященной данному вопросу статье Чанг [54]. Теорема Фреймана и полиномиальная гипотеза связаны также и с другой важной областью - обратными результатами для норм Гауэрса и их применениям в теории чисел (см. [10]-[15], [55], [56], а также раздел 8). Мы отметим здесь лишь два ярких теоретико-числовых приложения [14].

Теорема 5. Количество арифметических прогрессий длины четыре, составленных из простых чисел $p_{1}<p_{2}<p_{3}<p_{4} \leqslant N$, равно

$$
(1+o(1)) \mathfrak{S}_{1} \frac{N^{2}}{\log ^{4} N},
$$

¿əe

$$
\mathfrak{S}_{1}=\frac{3}{4} \prod_{p \geqslant 5}\left(1-\frac{3 p-1}{(p-1)^{3}}\right) .
$$

Как известно [57], простые числа содержат арифметические прогрессии любой длины. Теорема 5 дает асимптотическую формулу для числа прогрессий длины 4 (для прогрессий длины три этот результат был известен ранее, см. [58]-[60]). Случай более длинных прогрессий также разобран в [14], но он формулируется несколько более сложно, поэтому мы не включили его в настоящий обзор. Еще одно приложение связано с усилением теоремы Виноградова о суммах трех простых. 
Теорема 6. Пусть $N$ - нечетное число. Тогда число представлений $N$ в виде суммы $N=p_{1}+p_{2}+p_{3}$ трех простых таких, что $p_{1}-p_{2} \in \mathscr{P}-1$, равно

$$
(1+o(1)) \mathfrak{S}_{3} \frac{N^{2}}{\log ^{4} N},
$$

где

$$
\mathfrak{S}_{3}=\frac{1}{3} \prod_{p \geqslant 3, p \mid N^{3}-N}\left(1-\frac{p^{2}-4 p+1}{(p-1)^{4}}\right) \cdot \prod_{p \geqslant 3, p \nmid N^{3}-N}\left(1+\frac{4 p-1}{(p-1)^{4}}\right) .
$$

Доказательство полиномиальной гипотезы Фреймана-Ружи позволило бы продвинуться в оценке остаточных членов теорем 5, 6 (см. [15]). Пока о малое в формулах (25), (26) этих утверждений является неэффективным.

Даже более слабые результаты по теореме Фреймана в духе оценки (24) дают множество приложений к теории чисел, аддитивной комбинаторике и другим областям математики (см., например, обзор [27] и статью [36]). Общая схема использования утверждений типа теорем 3, 4 была предложена Гауэрсом в работе [8] (см. обсуждение в разделе 6 настоящей статьи), который успешно применил ее [8], [9] в получении первых количественных результатов по теореме Семереди об арифметических прогрессиях [61], [62] (см. также обзор о теореме Семереди [4]). Затем подобные рассуждения стали систематически использоваться различными авторами. Так, Семереди и Ву [63] применили ее в задачах об арифметических прогрессиях в суммах $l A, l$ - большое. Тао и Ву нашли приложение к теории случайных матриц [64]. Упомянем теперь несколько аддитивно-комбинаторных следствий. В статье [65] была доказана следующая теорема.

Теорема 7. Пусть $N, k$ - натуральные числа, $k \geqslant 6$. Пусть также $c_{1}, \ldots, c_{k}-$ любые целье числа такие, что $c_{1}+\cdots+c_{k}=0$. Наконеи, пусть $A \subseteq\{1,2, \ldots, N\}-$ произвольное множество без решений уравнения

$$
c_{1} x_{1}+\cdots+c_{k} x_{k}=0
$$

где $x_{1}, \ldots, x_{k} \in A-$ различные. Тогда

$$
|A| \ll \exp \left\{-\log ^{1 / 7} N\right\} N
$$

где константа с >0 зависит только от $c_{1}, \ldots, c_{k}$.

Полученный результат интересен тем, что он, с точностью до константы в степени логарифма, совпадает с нижней границей на мощность множеств, не содержащих решений уравнения (27) (см. [66]).

Вопросы об арифметических прогрессиях в суммах произвольных множеств составляют интенсивно развивающийся раздел аддитивной комбинаторики (см., например, обзоры [4], [33], а также работы [24], [25], [36], [67]-[73]). Из результатов Сандерса [37] вытекает следующая теорема. 
Теорема 8. Пусть $N$ - натуральное число и $\delta \in(0,1]$ - действительное число. Пусть $A_{1}, A_{2}, A_{3}, A_{4} \subseteq\{1,2, \ldots, N\}$ - произвольные множества мощности, не меньшей, чем $\delta N$. Тогда множество $A_{1}+A_{2}+A_{3}+A_{4}$ содержит арифметическую прогрессию длины

$$
N^{O\left(\log ^{-C} \delta^{-1}\right)},
$$

где $C>0$ - некоторая абсолютная константа.

Пользуясь результатами и методами работы [37], можно доказать теорему об оценке $L_{4}$-нормы тригонометрической суммы по квадратам (см. [27], [36]).

Теорема 9. Пусть $n_{1}<n_{2}<\cdots<n_{k}-$ натуральные числа. Тогда

$$
\int_{0}^{1}\left|\sum_{j=1}^{k} \exp \left\{2 \pi i n_{j}^{2} \alpha\right\}\right|^{4} d \alpha \ll k^{3} \exp \left\{-\log ^{c} k\right\},
$$

где $C, c>0$ - некоторые абсолютные константы.

Теорема 9 представляет собой продвижение на пути к замечательной гипотезе Рудина [74] о том, что множество квадратов составляет “почти" $\Lambda(4)$-множество. Иными словами, он предположил, что справедлива следующая гипотеза.

ГиПотеЗА 4. Пусть $n_{1}<n_{2}<\cdots<n_{k}-$ натуральные числа. Тогда

$$
\int_{0}^{1}\left|\sum_{j=1}^{k} \exp \left\{2 \pi i n_{j}^{2} \alpha\right\}\right|^{4} d \alpha \ll k^{2+o(1)} .
$$

Эти же идеи позволяют усилить результат из [75].

Теорема 10. Пусть $A \subset \mathbb{R} u \alpha-$ трансцендентное число. Тогда

$$
|A+\alpha \cdot A| \gg|A| \exp \left\{C \log ^{c}|A|\right\},
$$

где $C, c>0$ - некоторые абсолютные константы.

Поскольку в теореме 10 используется, так сказать, логарифмическая шкала, то оценка (28) особенно близка к верхней: как известно [75], существует $A \subset \mathbb{R}$, для которого $|A+\alpha \cdot A|=|A| \exp \left\{O\left(\log ^{1 / 2}|A|\right)\right\}$.

Совсем недавно обнаружилось, что данный подход может быть полезен и в задачах теории функций [76]-[80]. На этом мы заканчиваем список приложений теоремы Сандерса-Конягина.

Помимо полиномиальной гипотезы Фреймана-Ружи, существует еще более сильная гипотеза, которая называется полиномиальной гипотезой Боголюбо8a. Название предложено в работе [52], сам Боголюбов [81] получил первый результат в этом направлении. Ружа [49] начал использовать теоремы такого типа в задачах о множествах с малым удвоением и, в частности, показал, что результаты боголюбовского типа сильнее фреймановских. Имеется несколько вариантов этой гипотезы (отличающихся числом слагаемых, а также кратностью вхождения элементов в сумму), мы приведем лишь один (см. [65]), так называемый плотностной, вариант. 
ГиПотезА 5. Пусть группа $\mathbf{G}$ - конечная, $\delta \in(0,1)$ - действительное число и $A \subseteq \mathbf{G}$ - произвольное множество, $|A|=\delta|\mathbf{G}|$. Тогда найдется множество Бора $\mathscr{B}(\Gamma, \varepsilon) \subseteq 2 A-2 A$ такое, ито $|\Gamma| \ll \log (1 / \delta)$ u $\varepsilon \gg \log ^{-1}(1 / \delta)$.

Наилучший результат по полиномиальной гипотезе Боголюбова был получен в работе [37]. Мы формулируем его в авторских терминах, а именно через константы удвоения соответствующих множеств. Известно [2; гл. 5], что данный подход может быть выражен через плотностной и наоборот.

Теорема 11. Пусть $\mathbf{G}$ - любая абелева группа и $A, B \subseteq \mathbf{G}$ - произвольные конечные множества такие, что $|A+B| \leqslant K \min \{|A|,|B|\}$. Тогда $(A-A)+$ $(B-B)$ содержит точную обобщенную арифметическую прогрессию $M$ в группе $\mathbf{G}$ размера $\exp \{-h(K)\}|A+B|$ и размерности $d(K)$. Здесъ $d(K)=O\left(\log ^{6} K\right)$ $u h(K)=O\left(\log ^{6} K \cdot \log \log K\right)$.

Можно показать, что в терминах теоремы 11 полиномиальная гипотеза Боголюбова означает, что $d(K)=h(K)=O(\log K)$.

\section{5. Доказательство теоремы Фреймана и теоремы Сандерса в группе $\mathbb{F}_{2}^{n}$}

В этом разделе мы дадим короткое доказательство количественной формы теоремы Фреймана в группе $\mathbb{F}_{2}^{n}$, принадлежащее Руже [47], а также доказательство теоремы Сандерса в той же группе. Важность групп $\mathbb{F}_{p}^{n}$ и, в частности, $\mathbb{F}_{2}^{n}$ обсуждается в обзоре [3]. Как уже отмечалось ранее, такие группы обладают рядом преимуществ: во-первых, они очень просто устроены, во-вторых, множества Бора в них совпадают с подпространствами, а это важно для анализа Фурье, и, наконец, в-третьих, очень часто перенос решений комбинаторных задач с групп $\mathbb{F}_{p}^{n}$ на произвольные группы сопряжен лишь с техническими, а не идейными сложностями. Поэтому, чтобы продемонстрировать пружины доказательств теорем Фреймана и Сандерса, очень естественно начать с простейших групп $\mathbb{F}_{2}^{n}$.

Сформулируем один количественный вариант теоремы Фреймана в $\mathbb{F}_{2}^{n}$ (см. [39]).

Теорема 12. Пусть $A \subseteq \mathbb{F}_{2}^{n}$ - произвольное множество, $|A+A| \leqslant K|A|$. Тогда найдется подпространство $H \subseteq \mathbb{F}_{2}^{n}$, содержащее множество А и такое, чmo

$$
|H| \leqslant K \cdot 2^{K^{5}}|A|
$$

Сразу надо отметить, что оценки теоремы 12 не являются наилучшими (см., например, [82], [83]). Более того, в работах [52], [84] получены точные асимптотические формулы для верхней границы (29) на мощность подпространства $H$, которые имеют вид $|H|=2^{2 K+O(\log K)}|A|$ (в недавней статье [85] найдено точное значение для мощности (минимального) $H$ из теоремы 12). Нашей целью является не получение таких тонких результатов, а только лишь демонстрация основных идей, лежащих в основе метода. 
Легко видеть, что нижние оценки на мощность $H$ имеют экспоненциальный характер. Действительно, пусть множество $A \subseteq \mathbb{F}_{2}^{n}$ состоит из $k$ векторов стандартного базиса $A=\left\{e_{1}, \ldots, e_{k}\right\}$. Тогда

$$
|A+A|=\left(\begin{array}{l}
k \\
2
\end{array}\right)+1, \quad K=\frac{k}{2}+O(1)
$$

и любое подпространство $H$, содержащее $A$, имеет размерность не менее $k$, а значит, $|H| \geqslant 2^{k}=2^{2 K+O(\log K)}|A|$. Отсюда видно, что в случае группы $\mathbb{F}_{2}^{n}$ верхние оценки в теореме Фреймана $|H|=2^{2 K+O(\log K)}|A|$ совпадают с нижними. Случай $\mathbb{F}_{p}^{n}$ рассмотрен в работе [86].

Мы начнем доказательство теоремы 12 с общих замечаний относительно аддитивных множеств. Предположим, что множества $A, B \subseteq \mathbf{G}$ аддитивно близки в том смысле, что $|A-B|$ мало по сравнению со средним геометрическим мощностей $A$ и $B$, т. е. с величиной $(|A||B|)^{1 / 2}$. Далее, пусть множества $B$ и $C$ аддитивно близки в том же смысле. Можно ли утверждать, что тогда и множества $A, C$ аддитивно близки? Ответ на этот вопрос оказывается положительным, а именно, справедливо неравенство треугольника Ружи.

Лемма 2. Пусть $A, B, C \subseteq \mathbf{G}$ - произволъные множества. Тогда

$$
|C||A-B| \leqslant|A-C||B-C| .
$$

Иными словами, бормула $d(A, B)=\log \left(|A-B| /\left(|A|^{1 / 2}|B|^{1 / 2}\right)\right)$ задает псевдометрику на пространстве аддитивных множеств:

$$
d(A, B) \leqslant d(A, C)+d(C, B) .
$$

ДокАзАТЕльство. Для каждого элемента $x \in A-B$ выберем уникальную пару $a=a(x) \in A, b=b(x) \in B$ такую, что $x=a-b$. Если таких пар несколько, то возьмем одну из них. Рассмотрим отображение

$$
\varphi: C \times(A-B) \rightarrow A \times B-\Delta(C),
$$

задаваемое формулой $\varphi(c, a-b)=(a-c, b-c)$. Нам надо убедиться в том, что отображение $\varphi$ инъективно, и тогда формула (30) будет доказана. Но если $\varphi(c, a-b)=(a-c, b-c)=\left(a^{\prime}-c^{\prime}, b-c^{\prime}\right)=\varphi\left(c^{\prime}, a-b^{\prime}\right)$, то $a-b=a^{\prime}-b^{\prime}$, что противоречит уникальности пары $(a, b)$. Значит, $a^{\prime}=a, b^{\prime}=b$ и, следовательно, $c^{\prime}=c$. Лемма доказана.

Если в лемме 2 положить $A=A, B=A, C=-A$, мы получим

$$
|A-A| \leqslant \frac{|A+A|^{2}}{|A|},
$$

и, значит, разность множества контролируются суммой. Аналогичное неравенство - в котором сумма и разность переставлены местами - получить гораздо сложнее (см. доказательство формулы (33) ниже). Такой эффект происходит из-за того, что сумма множества устроена в некотором смысле хуже, чем разность, а значит, условие на малость суммы более сильное, чем условие на малость разности. 
Зададимся теперь другим вопросом. Предположим, что множество $A$ является множеством с малым удвоением. Верно ли, что его сумма или разность также будут множествами с малым удвоением? Это уже не совсем простой вопрос, но ответ на него тоже оказался положительным. Мы сформулируем замечательную теорему Плюннеке [87], переоткрытую и изложенную на современном языке Ружей [88]. Приведенное ниже элементарное доказательство принадлежит Петридису [89], [90].

Прежде всего определим коэфбициент растяжения для пары множеств $(A, B) \subseteq \mathbf{G}^{2}:$

$$
R_{B}[A]:=\min _{\varnothing \neq X \subseteq A} \frac{|B+X|}{|X|} .
$$

Смысл коэффициента растяжения очевиден: для всякого непустого множества $Y$ из $A$ сумма $|B+Y|$ будет больше $|Y|$ не менее чем в $R_{B}[A]$ раз. В случае $B=A$ легко видеть, что коэффициент растяжения не превосходит константы удвоения множества $A$.

Пусть минимум выше достигается на множестве $X$. Это множество обладает весьма замечательным свойством.

Лемма 3. Для произвольного множества $C \subseteq \mathbf{G}$ выполнено неравенство

$$
|B+X+C| \leqslant R_{B}[A]|X+C| .
$$

ДоКАЗАТЕЛЬСтво. Мы используем индукцию по мощности множества $C$. Для $|C|=1$ имеем $|B+X+C|=|B+X|=R_{B}[A]|X|=R_{B}[A]|X+C|$ и и утверждение леммы выполнено. Пусть теперь $C=C^{\prime} \sqcup\{c\}$ и

$$
Y=\left\{x \in X: x+c \subseteq X+C^{\prime}\right\} \subseteq X
$$

Тогда по определению множества $Y$ имеем

$$
B+X+C=\left(B+X+C^{\prime}\right) \cup((B+X+c) \backslash(B+Y+c)) .
$$

Следовательно, по предположению индукции, а также определению множества $X$ получаем

$|B+X+C| \leqslant\left|B+X+C^{\prime}\right|+|B+X|-|B+Y| \leqslant R_{B}[A]\left|X+C^{\prime}\right|+R_{B}[A]|X|-|B+Y|$.

Поскольку $Y \subseteq X$, то $|B+Y| \geqslant R_{B}[A]|Y|$. Соединяя это неравенство с оценкой (32) и еще раз используя определение множества $Y$, окончательно находим

$$
\begin{aligned}
|B+X+C| & \leqslant R_{B}[A]\left|X+C^{\prime}\right|+R_{B}[A](|X|-|Y|) \\
& =R_{B}[A]\left(\left|X+C^{\prime}\right|+|X \backslash Y|\right)=R_{B}[A]|X+C| .
\end{aligned}
$$

Лемма доказана. 
Из леммы 3 вытекает неравенство, противоположное (31) в том смысле, что плюс и минус в нем поменялись местами:

$$
|A+A| \leqslant \frac{|A-A|^{2}}{|A|} .
$$

В свою очередь, последняя оценка следует из варианта неравенства треугольника (30) с плюсами:

$$
|C||A+B| \leqslant|A+C||B+C| .
$$

Чтобы доказать (34), возьмем множество $X \subseteq C$ такое, что $|A+X|=|X| R_{A}[C]$. Тогда по лемме 3 имеем

$$
|A+B| \leqslant|A+B+X| \leqslant R_{A}[C]|B+X|=\frac{|A+X|}{|X|}|B+X| \leqslant \frac{|A+C|}{|C|}|B+C|,
$$

и неравенство (34) доказано.

Теперь сформулируем теорему Плюннеке-Ружи.

Теорема 13. Пусть $K \geqslant 1$ - действительное число, $A \subseteq \mathbf{G}$ - произвольное множество, $|A+A| \leqslant K|A|$. Тогда для любых натуральных чисел $n, m$ выполнено неравенство

$$
|n A-m A| \leqslant K^{n+m}|A| \text {. }
$$

Доказательство. Возьмем $X \subseteq A$ такое, что $|A+X|=|X| R_{A}[A]$. Применяя несколько раз лемму 3 , находим

$|n A+X|=|A+(n-1) A+X| \leqslant R_{A}[A]|(n-1) A+X| \leqslant \cdots \leqslant R_{A}^{n}[A]|X| \leqslant K^{n}|X|$

и, аналогично, $|m A+X| \leqslant K^{m}|X|$. Соединяя эти два неравенства и пользуясь неравенством треугольника, получаем

$$
|n A-m A| \leqslant \frac{|n A+X||m A+X|}{|X|} \leqslant K^{n+m}|X| \leqslant K^{n+m}|A| .
$$

Теорема доказана.

В частности, сумма или разность множества с малым удвоением также будут множествами с малым удвоением. Другие общие результаты о суммах множеств можно найти в [91].

Наконец, последняя необходимая нам лемма называется леммой Ружи о покрытии. Смысл этой леммы состоит в том, что если два множества $A$ и $B$ аддитивно близки, т. е. величина $|A+B|$ мала по сравнению с мощностями $A$ и $B$, то их структура должна быть схожей, а именно, каждое из множеств будет покрываться небольшим числом трансляций разности другого.

Лемма 4. Пусть $A, B \subseteq \mathbf{G}$ - произвольные множества. Тогда найдется множество $X,|X| \leqslant|A+B| /|A|$, такое, что

$$
B \subseteq A-A+X
$$


Доказательство этой леммы очевидно. Достаточно рассмотреть максимальную по включению систему множеств $A+x \subseteq A+B, x \in X \subseteq B$. Похожим образом доказывается один вариант леммы Чанг о покрытии [26], принадлежащий Сандерсу [37]. Напомним, что для множества $T=\left\{t_{1}, \ldots, t_{s}\right\}$ его $\operatorname{Span}(T)-$ это множество $\left\{\sum_{j=1}^{s} \varepsilon_{j} t_{j}: \varepsilon_{j} \in\{0, \pm 1\}\right\}$.

Лемма 5. Пусть $A, B \subseteq \mathbf{G}, k$ - натуральное число $u|A+k B| \leqslant 2^{k}|A|$. Тогда найдется множество $T,|T| \leqslant k$, такое, что

$$
B \subseteq A-A+\operatorname{Span}(T)
$$

Действительно, пусть $T \subseteq B, T=\left\{t_{1}, \ldots, t_{s}\right\},-$ максимальное такое, что множества

$$
A+\left\{\sum_{j=1}^{s} \varepsilon_{j} t_{j}: \varepsilon_{j} \in\{0,1\}\right\}
$$

не пересекаются. Ясно, что из максимальности $T$ вытекает справедливость включения $B \subseteq A-A+\operatorname{Span}(T)$. С другой стороны, если $|T|>k$, то взяв любое подмножество $T^{\prime} \subseteq T,\left|T^{\prime}\right|=k+1$, и положив

$$
P=\left\{\sum_{j=1}^{k+1} \varepsilon_{j} t_{j}^{\prime}: \varepsilon_{j} \in\{0,1\}\right\} \backslash\left\{\sum_{j=1}^{k+1} t_{j}^{\prime}\right\},
$$

так что $|P|=2^{k+1}-1$, получим противоречие

$$
\left(2^{k+1}-1\right)|A| \leqslant|P+A| \leqslant|k B+A| \leqslant 2^{k}|A| .
$$

Теперь мы можем приступить к доказательству теоремы Фреймана 12.

Без ограничения общности считаем, что $0 \in A$. Применим лемму 4 для множеств $A=A$ и $B=D+D$, где $D=A-A$ (в $\mathbb{F}_{2}^{n}$, конечно, неважно, что писать плюс или минус, но мы определяем множество $D$ таким образом, чтобы данные рассуждения сохранились и в случае произвольной группы). Тогда

$$
D+D \subseteq D+X
$$

где по теореме 13

$$
|X| \leqslant \frac{|D+D+A|}{|A|} \leqslant K^{5} .
$$

Из включения (36) вытекает, что минимальное подпространство $H(D)$, содержащее $D$, принадлежит $D+H(X)$. Следовательно, $A \subseteq D \subseteq H(D)$ и $|H(D)| \leqslant|D| \cdot 2^{|X|} \leqslant K \cdot 2^{K^{5}}|A|$, что и требовалось доказать.

Получим теперь симметричный случай теоремы Сандерса 11 в группе $\mathbb{F}_{2}^{n}$.

Теорема 14. Пусть $A \subseteq \mathbb{F}_{2}^{n}$ - произвольное множество с $\mathrm{D}[A]=K$. Тогда $2 A-2 A$ содержит подпространство $H$ размера $|H| \geqslant|A| \exp \left\{-O\left(\log ^{4} K\right.\right.$. $\log \log K)\}$. 
Прежде чем начать доказывать теорему 14, рассмотрим одно важное понятие. Множество $A \subseteq \mathbf{G}$ называется множеством полиномиалъного роста cmenени $d$, если для всех натуральных $n$ выполнено неравенство

$$
|n A| \leqslant n^{d}|A| \text {. }
$$

Известная теорема Хованского [92] (см. также элементарное доказательство в [93]) утверждает, что для любого конечного подмножества абелевой полугруппы найдется многочлен $p(n)$ такой, что $|n A|=p(n)$ для всех достаточно больших $n$. Таким образом, любое конечное множество обладает полиномиальным ростом, при этом степень $d$ может зависеть от этого множества. Нас, конечно, будут интересовать оценки, где, в некотором смысле, степень $d$ от множества не зависит, но может выражаться через некоторые его характеристики.

Покажем, например, что любое множество с константой удвоения $K$ является множеством полиномиального роста степени $O\left(K^{5}\right)$. По теореме 13 мы знаем, что для всех натуральных $n$ выполнено неравенство $|n A| \leqslant K^{n}|A|$, но это экспоненциальный рост и поэтому данной оценки недостаточно. С другой стороны, при доказательстве теоремы 12 мы видели, что для $D=A-A$ справедливо включение $(36)$, и, следовательно, $n D \subseteq(n-1) X+D$. Поскольку группа $\mathbf{G}$ абелева, то

$$
|(n-1) X| \leqslant\left(\begin{array}{c}
|X|+n-2 \\
|X|-1
\end{array}\right) \leqslant n^{|X|}
$$

и, значит, по неравенству Плюннеке и оценке выше, находим

$$
|n A| \leqslant|n D| \leqslant|(n-1) X||D| \leqslant K^{2}|A| n^{|X|} \leqslant n^{O\left(K^{5}\right)}|A| .
$$

Итак, любое множество с малым удвоением является множеством полиномиального роста со степенью, зависящей только от константы удвоения. По теореме Фреймана в $\mathbb{F}_{2}^{n}$ мы знаем, что произвольное множество с малым удвоением экономно покрывается некоторым подпространством (см. теорему 12). Для множеств с полиномиальным ростом последний результат можно легко доказать и непосредственно.

Лемма 6. Пусть $A \subseteq \mathbb{F}_{2}^{n}-$ произвольное множество полиномиального роста степени d. Тогда найдется подпространство $H$, содержащее $A$ и такое, чmo $|H| \leqslant \exp \{O(d \log d)\}|A|$.

Чтобы получить это утверждение, нам понадобится лемма Сандерса [94] (уточнения и обобщения этой леммы содержатся в статье [95]).

Лемма 7. Пусть $A, B \subseteq \mathbb{F}_{2}^{n}$ - произвольные множества, удовлетворяющие неравенству $|A+B| \leqslant K|A|$. Тогда $\operatorname{dim} B \ll K \log |A|$.

Для доказательства леммы 7 достаточно взять максимальное независимое множество $\Lambda \subseteq B$ и заметить, что, по теореме Плюннеке и известным оценкам для биномиальных коэффициентов, для любого натурального $k$ выполнены неравенства

$$
\frac{|\Lambda|^{k}}{(C k)^{k}} \leqslant\left(\begin{array}{c}
|\Lambda| \\
k
\end{array}\right) \leqslant|k \Lambda| \leqslant|k B| \leqslant K^{k}|A|,
$$


где $C>0$ - некоторая абсолютная константа. Выбирая теперь параметр $k$ равным $2+[\log |A|]$, находим $|\Lambda| \ll K \log |A|$, что и требовалось.

Имея лемму Сандерса 7, мы уже легко получаем лемму 6. Действительно, рассмотрим последовательность вложенных множеств

$$
A+A \subseteq 2 A+A \subseteq \cdots \subseteq(n-1) A+A=n A .
$$

Поскольку $|n A| \leqslant n^{d}|A|$, то по принципу Дирихле найдется $l=O(d \log d)$ такое, что $|l A|=|(l-1) A+A| \leqslant 2|(l-1) A|$. Но тогда по лемме 7 (более аккуратные рассуждения содержатся в $[37 ; \S 6]$, см. также [95]) имеют место оценки

$$
\operatorname{dim} A \ll \log |(l-1) A| \leqslant \log \left(l^{d}|A|\right) \leqslant \log |A|+d \log l \leqslant \log |A|+O(d \log d),
$$

что и требовалось.

Вооруженные концепцией полиномиального роста, мы можем теперь приступить к доказательству теоремы 14. В методе Сандерса используется сразу несколько идей. Самая главная из них - это изящное применение теоремы Крута-Сисаска о почти периодичности сверток (см. [24] и [25]). Этот результат интересен сам по себе, см., например, его приложение к задаче о множествах без арифметических прогрессий в работе [96], изложению из которой мы и следуем.

Теорема 15. Пусть $\epsilon \in(0,1), K \geqslant 1$ - действительные числа, $p$ - натуральное, $A, B \subseteq \mathbf{G}$ таковь, ито $|A+B| \leqslant K|A|$, и пусть $f \in L_{p}(\mathbf{G})$ произвольная функиия. Тогда найдутся $b \in B$ и множество $T \subseteq B,|T| \geqslant$ $|B|(2 K)^{-O\left(\epsilon^{-2} p\right)}$, такие, что для всех $t \in T-b$ выполнено неравенство

$$
\left\|\left(f * \mu_{A}\right)(x+t)-\left(f * \mu_{A}\right)(x)\right\|_{L_{p}(\mathbf{G}, x)} \leqslant \epsilon\|f\|_{L_{p}(\mathbf{G})} .
$$

ДокАЗАтЕЛьство. Пусть $k$ - натуральный параметр, $k=O\left(\epsilon^{-2} p\right)$. Выберем $k$ случайных точек $x_{1}, \ldots, x_{k} \in A$ равномерно и независимо и положим $X_{j}(y)=f\left(y+x_{j}\right)-\left(f * \mu_{A}\right)(y)$. Для любого фиксированного $y$ случайные величины $X_{j}(y)$ являются независимыми и имеют нулевое математическое ожидание и дисперсию, не превосходящую $\left(|f|^{2} * \mu_{A}\right)(y)$. По неравенству Хинчина для сумм независимых случайных величин получаем

$$
\left\|\sum_{j=1}^{k} X_{j}(y)\right\|_{L_{p}\left(\mu_{A}^{k}\right)} \ll\left(p k\left(|f|^{2} * \mu_{A}\right)(y)\right)^{1 / 2} .
$$

Возводя последнее неравенство в $p$-ю степень, деля на $k^{p}$, интегрируя по $y$ и применяя неравенство Гёльдера для $L_{p}(y-A)$, которое дает $\left(|f|^{2} * \mu_{A}\right)^{p / 2}(y) \leqslant$ $\left(|f|^{p} * \mu_{A}\right)(y)$, находим

$$
\iint\left|\frac{1}{k} \sum_{j=1}^{k} f\left(y+x_{j}\right)-\left(f * \mu_{A}\right)(y)\right|^{p} d y d \mu_{A}^{k}(\mathbf{x}) \ll\left(p k^{-1}\|f\|_{L_{p}(\mathbf{G})}^{2}\right)^{p / 2} .
$$

Здесь $\mathbf{x}=\left(x_{1}, \ldots, x_{k}\right)$. Снова используя неравенство Гёльдера и вспоминая, что $k=O\left(\epsilon^{-2} p\right)$, имеем

$$
\int\left\|\frac{1}{k} \sum_{j=1}^{k} f\left(y+x_{j}\right)-\left(f * \mu_{A}\right)(y)\right\|_{L_{p}(\mathbf{G}, y)} d \mu_{A}^{k}(\mathbf{x}) \leqslant \frac{\epsilon}{4}\|f\|_{L_{p}(\mathbf{G}) .}
$$


Из оценки (39) вытекает, что множество $L$, состоящее из $\mathbf{x} \in A^{k}$ таких, что подынтегральное выражение меньше $\epsilon\|f\|_{L_{p}(\mathbf{G})} / 2$, имеет меру $\mu_{A}^{k}(L) \geqslant 1 / 2$. Поскольку для $\Delta=\Delta(B)$ выполнено включение $\Delta+A \subseteq(A+B)^{k}$, то $|\Delta+L| \leqslant$ $2 K^{k}|L|$ и мы получаем из (13), что

$$
\sum_{\alpha, \beta \in \mathbf{G}^{k}} \Delta(\alpha) \Delta(\beta)(L \circ L)(\alpha-\beta)=\mathrm{E}(\Delta, L) \geqslant \frac{|\Delta|^{2}|L|}{2 K^{k}} .
$$

Следовательно, найдется $\beta \in \Delta, \beta=(b, \ldots, b), b \in B$, для которого выполнено неравенство

$$
|\{\alpha \in \Delta: \alpha-\beta \in L-L\}| \geqslant \frac{|\Delta|}{2 K^{k}} .
$$

Полагая $T=\{t \in B: \Delta(t)-\Delta(b) \in L-L\}$, мы видим, что $|T| \geqslant|B|(2 K)^{-O\left(\epsilon^{-2} p\right)}$. Кроме того, для всякого $s \in T-b$ имеем $\Delta(s) \in L-L$, и, следовательно, найдется $\alpha=\left(\alpha_{1}, \ldots, \alpha_{k}\right) \in L$, для которого $\alpha+\Delta(s)=\left(s+\alpha_{1}, \ldots, s+\alpha_{k}\right) \in L$. Значит, по неравенству треугольника

$$
\left\|\left(f * \mu_{A}\right)(x+t)-\left(f * \mu_{A}\right)(x)\right\|_{L_{p}(\mathbf{G}, x)} \leqslant \epsilon\|f\|_{L_{p}(\mathbf{G})},
$$

что и требовалось доказать.

Итак, теорема 15 показывает, что у свертки интегрируемой функции с характеристической функцией множества существует большое множество почти периодов $T-b$, причем почти периодичность понимается в $L_{p}$-смысле. Заметим также, что теорема Крута-Сисаска несимметрична по $A$ и $B$, можно представлять себе множество $A$ большим, а $B$ малым, например, $A=H \dot{+} \Lambda$, $B=H$ из примера 5. Другие результаты о почти периодичности собраны в [25]. Оптимальная версия теоремы Крута-Сисаска доказана в [21].

Следующая идея Сандерса - это старое аналитическое наблюдение, заключающееся в том, что взятие все больших сумм множеств существенно упрощает аддитивную задачу. Сандерс остроумно применил эту мысль при доказательстве теоремы 14. Другой важный момент состоит в использовании тождества (42).

СлеДСТВИЕ 1. Пусть $A, B, C \subseteq \mathbf{G}-$ произвольные множества, $|A+B| \leqslant$ $K|A|,|B+C| \leqslant L|B|$. Пусть также $\epsilon \in(0,1)$ - действительное число и $k$ натуральное. Тогда найдется множество $X \subseteq \mathbf{G}, X=-X, 0 \in X$, такое, yтo

$$
|X| \geqslant \exp \left\{-O\left(\epsilon^{-2} k^{2} \log K \log L\right)\right\}|C|
$$

и для всех $x \in k X$ выполнено неравенство

$$
|(A+B) \circ(A * B)(x)-| A|| B|| \leqslant \epsilon|A||B| .
$$

Следствие 1 мгновенно вытекает из теоремы 15 (подобные рассуждения использовались и в статьях [8], [9]). Действительно, поскольку (см. приложения этого тождества в [97], [98])

$$
\sum_{\alpha}(A+B)(\alpha)(A * B)(\alpha)=|A||B|,
$$


то формула (41), очевидно, справедлива для $x=0$. Далее, применяя теорему 15 с $A=-B, f=A+B$ и пользуясь неравенством треугольника, получаем, что найдется симметричное множество $X,|X| \geqslant \exp \left\{O\left(\epsilon^{-2} k^{2} p \log L\right)\right\}|C|$, такое, что для всех $x \in k X$ выполнено неравенство

$$
\left\|\left((A+B) \circ \mu_{B}\right)(y+x)-\left((A+B) \circ \mu_{B}\right)(y)\right\|_{L_{p}(\mathbf{G}, y)} \leqslant \epsilon e^{-1}\|(A+B)\|_{L_{p}(\mathbf{G})} .
$$

Здесь $p$ - параметр, который мы выбираем равным $2+[\log K]$. Соединяя почти периодичность (43) с тождеством (42) и используя неравенство Гёльдера, получаем, что для всех $x \in k X$ справедлива оценка

$$
\begin{aligned}
& |((A+B) \circ(A * B))(x)-| A|| B|| \\
& \quad \leqslant\|A\|_{L_{p^{\prime}}(\mathbf{G})} \cdot\|((A+B) \circ B)(y+x)-((A+B) \circ B)(y)\|_{L_{p}(\mathbf{G}, y)} \\
& \quad \leqslant \epsilon e^{-1}|A|^{1 / p^{\prime}}|A+B|^{1 / p}|B| \leqslant \epsilon e^{-1} K^{1 / p}|A||B| \leqslant \epsilon|A||B| .
\end{aligned}
$$

Поскольку от множества почти периодов мы перешли к сумме $k X$, которая по формуле (41) также является множеством почти периодов, то мы можем применить наш аналитический трюк. Используя следствие 1 с $B=C=A$, $L=K$ и вычисляя преобразование Фурье, находим

$$
\begin{aligned}
\sum_{x}(2 A \circ(A * A))(x)\left(X *_{k-1} X\right)(x) & =\frac{1}{2^{n}} \sum_{\xi} \widehat{(2 A)}(\xi) \widehat{A}^{2}(\xi) \widehat{X}^{k}(\xi) \\
& \geqslant(1-\epsilon)|A|^{2}|X|^{k} .
\end{aligned}
$$

Чтобы разобраться в том, какие слагаемые вносят существенный вклад в формулу (44), нам понадобится важное понятие спектра множества $A \subseteq \mathbf{G}$. Пусть $c \in(0,1]$ - произвольное действительное число. Тогда $\operatorname{Spec}_{c}(A)$ (или множество больших тригонометрических сумм множества $A$ ) - это

$$
\operatorname{Spec}_{c}(A)=\{\xi \in \widehat{\mathbf{G}}:|\widehat{A}(\xi)| \geqslant c|A|\} .
$$

Такие множества интенсивно изучались в работах [50], [67], [97], [99]-[104], см. также обзор [33]. Замечательным результатом теории больших тригонометрических сумм, имеющим множество приложений (см., например, [26], [36], [72], [105]), является теорема Чанг [26] (простое доказательство может быть найдено в [101], [103]), которую мы формулируем здесь в частном случае $\mathbf{G}=\mathbb{F}_{2}^{n}$.

Теорема 16. Пусть $X \subseteq \mathbb{F}_{2}^{n}$ - произвольное множество и $с \in(0,1]$ - некоторое число. Тогда

$$
\operatorname{dim}\left(\operatorname{Spec}_{c}(X)\right) \ll c^{-2} \log \frac{2^{n}}{|X|} .
$$

Выберем параметр $k$ равным $2+\left[\log \left(\epsilon^{-1} K\right)\right]$, а $\epsilon$ будет взято впоследствии равным $1 / 4$. Тогда ясно, что в правой части равенства (44a) существенный 
вклад вносят лишь $\xi$, которые принадлежат $\operatorname{Spec}_{c}(X)$, где $c$ равно, скажем, 1/2. Действительно, применяя равенство Парсеваля, мы имеем

$$
\begin{aligned}
\frac{1}{2^{n}} \sum_{\xi \notin \operatorname{Spec}_{c}(X)}|\widehat{(2 A)}(\xi)| \widehat{A}^{2}(\xi)|\widehat{X}|^{k}(\xi) & <(c|X|)^{k} \frac{1}{2^{n}} \sum_{\xi}|\widehat{(2 A)}(\xi)| \widehat{A}^{2}(\xi) \\
& \leqslant(c|X|)^{k}|2 A||A| \leqslant K(c|X|)^{k}|A|^{2} \leqslant \epsilon|A|^{2}|X|^{k} .
\end{aligned}
$$

Следовательно, если $\mathscr{L}$ - это минимальное подпространство, содержащее множество $\operatorname{Spec}_{c}(X)$, то

$$
\frac{1}{2^{n}} \sum_{\xi \in \mathscr{L}} \widehat{(2 A)}(\xi) \widehat{A}^{2}(\xi) \widehat{X}^{k}(\xi) \geqslant(1-2 \epsilon)|A|^{2}|X|^{k} .
$$

Пусть $H=\mathscr{L}^{\perp}$. Пользуясь тождеством (10) и переходя, как в формуле (44), от преобразований Фурье обратно в “физическое пространство” $\mathbf{G}$, получаем

$$
\sum_{x}(2 A \circ(A * A))(x)\left(\left(X *_{k-1} X\right) * \mu_{H}\right)(x) \geqslant(1-2 \epsilon)|A|^{2}|X|^{k} .
$$

Отсюда и из соображений среднего находим $x$ такое, что $|2 A \cap(H+x)| \geqslant$ $(1-2 \epsilon)|H|$. Но тогда, очевидно, $H \subseteq 2 A-2 A$, поскольку мы выбрали $\epsilon=$ $1 / 4$, и, значит, нужное нам включение доказано. Осталось получить оценку $|H| \geqslant|A| \exp \left\{-O\left(\log ^{4} K \cdot \log \log K\right)\right\}$ или, ввиду равенства $\operatorname{dim} H=n-\operatorname{dim} \mathscr{L}$, найти подходящую верхнюю границу на размерность подпространства $\mathscr{L}$. Если бы мы рассматривали более простой плотностной случай, то для оценки этой размерности мы бы просто применили теорему Чанг и неравенство $|X| \geqslant \exp \left\{-O\left(\log ^{4} K\right)\right\}|A|$. В общей ситуации нам необходимо доказать, что множество $X$ обладает полиномиальным ростом степени $d=O\left(\log ^{4} K\right)$, а затем воспользоваться леммой 6 и теоремой 16. Действительно, если $X$ имеет полиномиальный рост, то по лемме 6 множество $X$ принадлежит некоторому подпространству $H_{*},\left|H_{*}\right| \ll \exp \{d \log d\}|X|$, и, следовательно,

$|H|=2^{\operatorname{dim} H}=2^{\operatorname{dim} H_{*}-\operatorname{dim} \mathscr{L}}=2^{\log |X|-O(d \log d)} \geqslant|A| \exp \left\{-O\left(\log ^{4} K \cdot \log \log K\right)\right\}$,

что и требуется. Наконец, полиномиальный рост множества $X$ вытекает из следующих соображений. По формуле (41) имеем $k X \subseteq 2 A-2 A$, где $k$ - достаточно большое такое, что $k \ll \log K$. Пусть $l$ - достаточно малый натуральный параметр со свойством $l \gg \log ^{3} K$. Тогда по неравенству Плюннеке

$$
|3 l k X| \leqslant|3 l(2 A-2 A)| \leqslant K^{12 l}|A| \leqslant|X| \exp \left\{O\left(\log ^{4} K+l \log K\right)\right\} \leqslant 2^{l k-2}|X| .
$$

Применяя лемму 5 с $A=X, B=3 X$ и $k=l k-1$, получаем, что $3 X \subseteq$ $2 X+\operatorname{Span}(T)$, где $|T| \leqslant l k \ll \log ^{4} K$. Отсюда, как в доказательстве теоремы 12 , имеем для всех натуральных чисел $n$

$$
\begin{aligned}
|n X| & \leqslant|(n+1) X| \leqslant|2 X+(n-1) \operatorname{Span}(T)| \leqslant|2 X| n^{|T|} \\
& \leqslant|2 A-2 A| n^{O\left(\log ^{4} K\right)} \leqslant \exp \left\{O\left(\log ^{4} K\right)\right\}|X| n^{O\left(\log ^{4} K\right)} \leqslant n^{O\left(\log ^{4} K\right)}|X|
\end{aligned}
$$

и, значит, множество почти периодов $X$ имеет полиномиальный рост степени $d=O\left(\log ^{4} K\right)$. 


\section{6. Теорема Балога-Семереди-Гауэрса}

В этом разделе мы обсудим важный аддитивно-комбинаторный результат теорему Балога-Семереди-Гауэрса и проиллюстрируем ее применение на примере задачи о суммах произведений в конечном поле [106]. Другое ее приложение обсуждается в разделе 8 (см. теорему 31). Часто теорему Балога-Семереди-Гауэрса применяют совместно с теоремой Фреймана (см. раздел 4). Примерами такого использования являются задачи о множествах без арифметических прогрессий [8], [9], проблемы, связанные с суммами произведений [107], вопросы теории функций [80], [108] и многие другие.

На данный момент самое короткое доказательство теоремы Балога-Семереди-Гауэрса принадлежит Шоену [109]. Замечательной особенностью его метода является явное построение множества $A_{*}$ с малой константой удвоения: таким множеством будет большое подмножество множества $A_{s}=A \cap(A-s)$ для случайного сдвига $s \in A-A$. С другой стороны, пример "картошек Шоена" указывает также и на слабость приводимого ниже доказательства теоремы 17. Действительно, в примере 6 структурированными объектами являются отнюдь не множества $A_{s}$, а сдвиги соответствующего подпространства $H$.

Теорема 17. Пусть $A \subseteq \mathbf{G}$ - произвольное множество, $K \geqslant 1$ - действительное число. Предположим, что $\mathrm{E}(A) \geqslant|A|^{3} / K$. Тогда найдется множество $A_{*} \subseteq A$ такое, что $\left|A_{*}\right| \geqslant|A| /(2 K)$ u

$$
\left|A_{*}-A_{*}\right| \leqslant 2^{14} K^{4}\left|A_{*}\right| .
$$

ДокАЗАТЕЛЬСтво. Пусть $a=|A|, \mathrm{E}=\mathrm{E}(A)=a^{3} / K$. Напомним, что мы пользуемся обозначением $A_{s}=A \cap(A-s)$. Имеем

$$
\mathrm{E}=\sum_{s}\left|A_{s}\right|^{2}=\sum_{s} \sum_{x, y} A_{s}(x) A_{s}(y)=\sum_{s} \sum_{x, y} A(x) A(y) A(x+s) A(y+s) .
$$

Пусть $\varepsilon \in(0,1)$ - некоторое число, которое мы выберем позднее; положим

$$
P_{\varepsilon}=\left\{s:\left|A_{s}\right| \geqslant \frac{\varepsilon a}{2 K}\right\} \text {. }
$$

Ясно, что для любого $\varepsilon$ множество $P_{\varepsilon}$ симметрично. Поскольку

$$
\sum_{s} \sum_{x-y \notin P_{\varepsilon}} A_{s}(x) A_{s}(y)=\sum_{x-y \notin P_{\varepsilon}} A(x) A(y)\left|A_{x-y}\right| \leqslant \frac{\varepsilon a^{3}}{2 K},
$$

то, соединяя последнюю оценку с (47), мы получаем

$$
\sum_{s:\left|A_{s}\right| \geqslant a /(2 K)} \sum_{x-y \in P_{\varepsilon}} A_{s}(x) A_{s}(y)-\varepsilon^{-1} \sum_{s:\left|A_{s}\right| \geqslant a /(2 K)} \sum_{x-y \notin P_{\varepsilon}} A_{s}(x) A_{s}(y)>0 .
$$


Значит, найдется $s$ такое, что $\left|A_{s}\right| \geqslant a /(2 K)$ и одновременно

$$
\varepsilon \sum_{x-y \in P_{\varepsilon}} A_{s}(x) A_{s}(y)>\sum_{x-y \notin P_{\varepsilon}} A_{s}(x) A_{s}(y) .
$$

Иными словами,

$$
\sum_{x-y \in P_{\varepsilon}} A_{s}(x) A_{s}(y)>(1-\varepsilon)\left|A_{s}\right|^{2} .
$$

Теперь рассмотрим неориентируемый граф $G=(V, E)$, множество вершин которого есть $A_{s}$ и вершины $x, y$ которого соединены ребром тогда и только тогда, когда $x-y \in P_{\varepsilon}$. Ясно, что $|E|>(1-\varepsilon)\left|A_{s}\right|^{2}$. Положим

$$
A_{*}=\{v \in V: \operatorname{deg} v \geqslant(1-2 \varepsilon)|V|\} \subseteq A_{s} .
$$

Из (48) вытекает оценка $\left|A_{*}\right| \geqslant \varepsilon|V|$. Кроме того, у любых вершин $a, b \in A_{*}$ найдется не менее $(1-4 \varepsilon)|V|$ общих соседей $x \in V$, т. е. вершин $x$ таких, что $(a, x) \in E$ и $(x, b) \in E$. Выберем $\varepsilon$ равным $1 / 8$. Пользуясь тождеством

$$
a-b=(a-x)+(x-b),
$$

а также определением множества $P_{\varepsilon}$, мы видим, что для любых $a, b \in A_{*}$ выполнено неравенство

$$
((A \circ A) *(A \circ A))(a-b) \geqslant\left(\frac{\varepsilon a}{2 K}\right)^{2} \frac{\left|A_{s}\right|}{2} .
$$

Следовательно,

$$
\left(\frac{\varepsilon a}{2 K}\right)^{2} \frac{\left|A_{s}\right|}{2}\left|A_{*}-A_{*}\right| \leqslant \sum_{z \in A_{*}-A_{*}}((A \circ A) *(A \circ A))(z) \leqslant \sum_{z}((A \circ A) *(A \circ A))(z)=a^{4} .
$$

Другими словами,

$$
\left|A_{*}-A_{*}\right| \leqslant 2^{9} K^{2} a^{2}\left|A_{s}\right|^{-1} \leqslant 2^{12} K^{2} a^{2}\left|A_{*}\right|^{-1} \leqslant 2^{14} K^{4}\left|A_{*}\right| .
$$

Теорема доказана.

Таким образом, теорему Балога-Семереди-Гауэрса можно рассматривать как результат о структуре множеств $A$, имеющих экстремальное (в терминах мощности или, другими словами, энергии $\left.\mathrm{E}_{1}(A)\right)$ значение аддитивной энергии $\mathrm{E}(A)$. Любое такое множество, говорит теорема, содержит большое подмножество $A_{*}$, имеющее малую (в терминах $K$ ) константу удвоения. Строение же такого рода множеств обсуждалось нами в разделе 4. С другой стороны, из неравенства Коши-Буняковского (13) легко вытекает, что произвольное множество $A$, имеющее подмножество $A_{*}$ с малым удвоением (46), автоматически обладает полиномиально большой аддитивной энергией $\mathrm{E}(A) \gg_{K}|A|^{3}$. Таким образом, теорема 17 , помимо того что она позволяет находить в $A$ подмножества с достаточно жесткой структурой, еще и является критерием того, что множество $A$ обладает большой (в терминах $\left.\mathrm{E}_{1}(A)\right)$ аддитивной энергией, 
а именно, соотношение $\mathrm{E}(A) \sim_{K}|A|^{3} \sim_{K}\left(\mathrm{E}_{1}(A)\right)^{3 / 2}$ возможно тогда и только тогда, когда для некоторого подмножества $A_{*} \subseteq A, A_{*} \gg_{K}|A|$, выполнено неравенство $\left|A_{*}-A_{*}\right| \ll_{K}\left|A_{*}\right|$.

Показатель четыре в теореме 17 можно немного улучшить, если частично пожертвовать симметрией (см. [109]). Дело в том, что доказательство неявно использует энергию $\mathrm{E}_{3}$, поскольку, как можно легко показать, обобщая рассуждения выше, для любого натурального $k$ справедливо неравенство $\left|A_{*}-A_{*}\right| \ll$ $\mathrm{E}_{k}^{2}(A) \mathrm{E}_{k+1}^{-4}(A)|A|^{2 k+6}\left|A_{*}\right|$ и, в частности, $\left|A_{*}-A_{*}\right| \ll \mathrm{E}^{2}(A) \mathrm{E}_{3}^{-4}(A)|A|^{10}\left|A_{*}\right|$ (выбор множества $A_{*}$ зависит от параметра $k$ ). Это, в свою очередь, означает, что константа 4 получается, только если соотношение между $\mathrm{E}_{3}$ и $\mathrm{E}$ экстремально, т. е. если $\mathrm{E}_{3}(A) \ll \mathrm{E}^{2}(A)|A|^{-2}$. Из последнего же неравенства вытекает, что у $A$ имеются сильные структурные свойства (более подробно см. раздел 7).

ТЕОрема 18. Пусть $A \subseteq \mathbf{G}-$ произвольное множество, $K \geqslant 1$ - действительное число. Предположим, что $\mathrm{E}(A) \geqslant|A|^{3} / K$. Тогда найдутся множества $A^{\prime}, B^{\prime} \subseteq A$ mакие, что $\left|A^{\prime}\right|,\left|B^{\prime}\right| \gg|A| K^{-3 / 4} \log ^{-5 / 4} K u$

$$
\left|A^{\prime}-B^{\prime}\right| \ll K^{7 / 2} \log ^{5 / 2} K \cdot\left(\left|A^{\prime}\right|\left|B^{\prime}\right|\right)^{1 / 2} .
$$

В работе [110] был доказан другой вариант теоремы Балога-Семереди-Гауэрса.

Теорема 19. Пусть $A \subseteq \mathbf{G}-$ произвольное множество, $K \geqslant 1$ - действительное число. Предположим, что $\mathrm{E}(A) \geqslant|A|^{3} / K$. Тогда найдется множество $A_{*} \subseteq$ A такое, что $\left|A_{*}\right| \gg|A| / K u$

$$
\left|A_{*}-A_{*}\right| \ll K^{4} \frac{\left|A_{*}\right|^{3}}{|A|^{2}} .
$$

Таким образом, теорема 17 хорошо работает, когда множество $A_{*}$ велико, а теорема 19 - когда $A_{*}$ мало.

Наконец, первая часть теоремы 23 раздела 7 ниже является примером "оптимальной" формы теоремы Балога-Семереди-Гауэрса, в том смысле, что если величина $M$ мала, то оценки на мощность множества $A^{\prime}$, а также на величину $\left|A^{\prime}-A^{\prime}\right|$ (случай $n=m=1$ ) являются наилучшими из возможных. Заметим, что впервые такого рода результат был доказан в статье [21] при несколько более сильных условиях.

Важность теоремы Балога-Семереди-Гауэрса заключается в том, что она, образно говоря, связывает через аддитивную характеристику множества $\mathrm{E}(A)$ два мира - комбинаторный и аналитический. Действительно, с одной стороны, и в этом, собственно, состоит утверждение теоремы, большая аддитивная энергия означает, что у множества есть подмножество с малым удвоением, а это чисто комбинаторное свойство. С другой стороны, из формулы (12), а именно

$$
\mathrm{E}(A)=\frac{1}{|\mathbf{G}|} \sum_{\xi}|\widehat{A}(\xi)|^{4},
$$

видно, что аддитивная энергия связана с тригонометрическими суммами по множеству $A$, т. е. с коэффициентами Фурье $A$, поскольку она равна $L_{4}$-норме 
преобразования Фурье характеристической функции. Если мы можем что-то сказать об аддитивной энергии, то это поможет нам оценить соответствующую тригонометрическую сумму. Еще одной замечательной особенностью теоремы является то, что все оценки в ней носят полиномиальный (по $K$ ) характер. Конечно, это также очень важно для приложений.

Для простоты мы рассматривали самый простой симметричный случай энергии $\mathrm{E}(A, A)$. В несимметричной ситуации справедлив следующий аналог теоремы 17 (см., например, [2; § 2.5]).

Tеорема 20. Пусть $A, B \subseteq \mathbf{G}$ - произвольные множества, $K \geqslant 1$ - действительное число. Предположим, что $\mathrm{E}(A, B) \geqslant(|A||B|)^{3 / 2} / K$. Тогда найдутся множества $A^{\prime} \subseteq A, B^{\prime} \subseteq B$ такие, что $\left|A^{\prime}\right| \gg|A| / K,\left|B^{\prime}\right| \gg|B| / K u$

$$
\left|A^{\prime}+B^{\prime}\right| \ll K^{C}(|A||B|)^{1 / 2},
$$

где $C>0$ - некоторая абсолютная константа.

Мы продемонстрируем применение теоремы Балога-Семереди-Гауэрса на примере замечательного результата из теории сумм произведений (см. пионерские работы [111]-[113], а также книгу [2; гл. 8]), дающего оценку тригонометрических сумм по очень маленьким мультипликативным подгруппам [114]. Ранее такие оценки были недостижимы для аналитических методов (см. [31]). Читателю, желающему быстро разобраться в вопросах сумм произведений, мы рекомендуем замечательную статью Грина [115], изложению из которой мы и следуем.

Теорема 21. Пусть $\delta \in(0,1]$ - действительное число, $p$ - простое число $u \Gamma \subseteq \mathbb{Z}_{p}^{*}-$ произвольная мультипликативная подгруппа, $|\Gamma| \geqslant p^{\delta}$. Тогда найдется $\varepsilon=\varepsilon(\delta)>0$ такое, что для всех $\xi \neq 0$ выполнено неравенство

$$
\left|\sum_{x \in \Gamma} e^{2 \pi i x \xi / p}\right| \ll|\Gamma| p^{-\varepsilon} .
$$

Теорема 21 доказывается рассуждением от противного. Предположим, что для какого-то $\xi$ неравенство (51)несправедливо. В терминах спектра $\Gamma$ (см. формулу (45)) это означает, что $\xi \in \operatorname{Spec}_{c}(\Gamma), c=p^{-\varepsilon}$, т. е. множество $\operatorname{Spec}_{c}(\Gamma)$ содержит ненулевой элемент. Мультипликативные подгруппы обладают характеристическим свойством Г-инвариантности, которое означает, что для всех $\gamma \in \Gamma$ выполнено равенство $\gamma \Gamma=\Gamma$. Ясно, что множество $\operatorname{Spec}_{c}(\Gamma)$ также является Г-инвариантным, т. е. для любого $\gamma \in \Gamma$ имеем $\gamma \operatorname{Spec}_{c}(\Gamma)=\operatorname{Spec}_{c}(\Gamma)$. Следовательно, если неравенство (51) не справедливо, то $\left|\operatorname{Spec}_{c}(\Gamma)\right|>|\Gamma|$. Нам понадобится один общий результат о спектре множества (см., например, [33; предложение 4]).

Лемма 8. Пусть $A \subseteq \mathbf{G}-$ произвольное множество,,$\in(0,1]$ - действительное число. Тогда для любого подмножества $B \subseteq \operatorname{Spec}_{c}(A)$ выполнено неравенство

$$
\sum_{x}(B \circ B)(x) \operatorname{Spec}_{c^{2} / 2}(A)(x) \geqslant 2^{-1} c^{2}|B|^{2} .
$$


Для всякого $\alpha \in(0,1)$ будем для краткости писать $S_{\alpha}$ вместо $\operatorname{Spec}_{\alpha}(\Gamma)$. Из неравенства Коши-Буняковского и оценки (52) имеем

$$
\mathrm{E}\left(S_{\alpha}, \gamma S_{\alpha}\right)=\mathrm{E}\left(S_{\alpha}\right) \gg \frac{c^{4}\left|S_{\alpha}\right|^{3}}{K},
$$

где $K=\left|S_{\alpha^{2} / 2}\right| /\left|S_{\alpha}\right| \geqslant 1$, а $\gamma \in \Gamma$ - произвольное. Мы уже получили условие теоремы Балога-Семереди-Гауэрса, однако ее эффективное применение подразумевает, конечно, что параметр $K$ достаточно мал. Этого можно легко добиться, рассмотрев вложенные множества

$$
S_{\alpha_{0}} \subseteq S_{\alpha_{1}} \subseteq \cdots \subseteq S_{\alpha_{J}}
$$

где $\alpha_{0}=c, \alpha_{j+1}=\alpha_{j}^{2} / 2$ и $J$ - некоторый параметр, который мы выберем позже. По принципу Дирихле найдется $j \in[J]$ такое, что $\left|S_{\alpha_{j+1}}\right| \leqslant p^{1 / J}\left|S_{\alpha_{j}}\right|$. Положим $\alpha=\alpha_{j}$. Тогда в силу оценки (53) для всех $\gamma \in \Gamma$ имеем

$$
\mathrm{E}\left(S_{\alpha}, \gamma S_{\alpha}\right) \gg\left(\alpha p^{-1 / J}\right)^{C}\left|S_{\alpha}\right|^{3}:=\frac{\left|S_{\alpha}\right|^{3}}{K},
$$

где $C>0$ - некоторая абсолютная константа. Ясно также, что $\alpha \geqslant(c / 2)^{2^{J}}$.

$\mathrm{K}$ каждой паре множеств $\left(S_{\alpha}, \gamma S_{\alpha}\right)$ мы можем применить теорему Балога-Семереди-Гауэрса 20, которая даст нам большие (в терминах $K$ ) подмножества $A^{\prime}, B^{\prime} \subseteq S_{\alpha}$ такие, что сумма $A^{\prime}+\gamma B^{\prime}$ мала. При этом множества $A^{\prime}, B^{\prime}$, конечно, зависят от вычета $\gamma$. С помощью несложных комбинаторных рассуждений мы избавимся от этой зависимости. Нам понадобится простая лемма, доказательство которой мы оставляем читателю (можно, например, использовать вероятностный метод [32]).

Лемма 9. Пусть $\delta \in(0,1]$ - действительное число и $M_{1}, \ldots, M_{k}$ - произвольные подмножества множества $M$ со свойством $\left|M_{j}\right| \geqslant \delta|M|, j \in[k]$. Тогда найдется $i \in[k]$ такое, что неравенство $\left|M_{i} \cap M_{j}\right| \geqslant \delta^{2}|M| / 2$ справедливо для не менее чем $\delta^{2} k / 2$ значений $j$.

Очень удобно использовать запись $A \approx_{K} B$, которая будет означать, что $d(A, B) \leqslant(\log K)^{\widetilde{C}}$, где $\widetilde{C}>0$ - некоторая абсолютная константа. Например, по теореме Балога-Семереди-Гауэрса 20 мы знаем, что $A^{\prime}(\gamma) \approx_{K} \gamma B^{\prime}(\gamma)$. Применяя теперь лемму 9 с $M=S_{\alpha} \times S_{\alpha}$ и $M_{\gamma}=A^{\prime}(\gamma) \times B^{\prime}(\gamma), \gamma \in \Gamma$, находим $\gamma_{0}$ и множество $X_{0} \subseteq \Gamma,\left|X_{0}\right| \gg|\Gamma| / K^{2}$ такие, что для всех $\gamma \in X_{0}$ выполнены неравенства

$$
\left|A^{\prime}(\gamma) \cap A^{\prime}\left(\gamma_{0}\right)\right| \gg \frac{\left|S_{\alpha}\right|}{K^{2}}, \quad\left|B^{\prime}(\gamma) \cap B^{\prime}\left(\gamma_{0}\right)\right| \gg \frac{\left|S_{\alpha}\right|}{K^{2}} .
$$

Кроме того, $A^{\prime}(\gamma) \approx_{K} \gamma B^{\prime}(\gamma), A^{\prime}\left(\gamma_{0}\right) \approx_{K} \gamma B^{\prime}\left(\gamma_{0}\right)$. По неравенству треугольника Ружи $A^{\prime}(\gamma) \approx_{K} A^{\prime}(\gamma), B^{\prime}(\gamma) \approx_{K} B^{\prime}(\gamma)$ и, кроме того,

$$
\begin{aligned}
& \left|A^{\prime}(\gamma) \cap A^{\prime}\left(\gamma_{0}\right)\right|\left|A^{\prime}(\gamma)-A^{\prime}\left(\gamma_{0}\right)\right| \leqslant\left|A^{\prime}(\gamma)-\left(A^{\prime}(\gamma) \cap A^{\prime}\left(\gamma_{0}\right)\right)\right| \\
& \quad \times\left|A^{\prime}\left(\gamma_{0}\right)-\left(A^{\prime}(\gamma) \cap A^{\prime}\left(\gamma_{0}\right)\right)\right| \leqslant\left|A^{\prime}(\gamma)-A^{\prime}(\gamma)\right|\left|A^{\prime}\left(\gamma_{0}\right)-A^{\prime}\left(\gamma_{0}\right)\right| .
\end{aligned}
$$


Значит, $A^{\prime}(\gamma) \approx_{K} A^{\prime}\left(\gamma_{0}\right), B^{\prime}(\gamma) \approx_{K} B^{\prime}\left(\gamma_{0}\right)$. Отсюда $\gamma B^{\prime}(\gamma) \approx_{K} \gamma B^{\prime}\left(\gamma_{0}\right)$ и, следовательно,

$$
A^{\prime}\left(\gamma_{0}\right) \approx_{K} A^{\prime}(\gamma) \approx_{K} \gamma B^{\prime}(\gamma) \approx_{K} \gamma B^{\prime}\left(\gamma_{0}\right) \approx_{K} \frac{\gamma}{\gamma_{0}} A^{\prime}\left(\gamma_{0}\right)
$$

Итак, мы нашли множество $X \subseteq \gamma_{0}^{-1} \Gamma,|X| \geqslant|\Gamma| / K^{C}$, и множество $A^{\prime} \subseteq S_{\alpha}$, $\left|A^{\prime}\right| \geqslant\left|S_{\alpha}\right| / K^{C}$, такие, что для всех $x \in X$ выполнено неравенство $\left|A^{\prime}+x A^{\prime}\right| \leqslant$ $K^{C}\left|A^{\prime}\right|$. Последний нужный нам инструмент - это феномен сумм произведений в $\mathbb{F}_{p}$ (см. книгу [2; гл. 8] и статью [106], явные оценки содержатся, например, в [116]), который вступает в противоречие с неравенствами для множеств $X$ и $A^{\prime}$, если выбрать параметр $J$ достаточно большим, а $\varepsilon(\delta)>0$ малым.

Tеорема 22. Пусть $A, B \subseteq \mathbb{F}_{p}$ - произволъные множества, $\alpha, \beta \in(0,1)$ некоторые числа, $p^{\alpha} \leqslant|A| \leqslant p^{1-\alpha},|B| \geqslant p^{\beta}$. Тогда найдется $b \in B$ такое, что $|A+b A| \geqslant|A|^{1+c(\alpha, \beta)}, c(\alpha, \beta)>0$.

Приведенный пример использования теоремы Балога-Семереди-Гауэрса наглядно показывает основной способ применения этой теоремы: сведение аналитики к комбинаторике, а именно, сведение аналитической оценки тригонометрической суммы (21) к чисто комбинаторному утверждению - теореме 22 .

Несколько иной взгляд на теорему Балога-Семереди-Гауэрса будет предложен в следующем разделе 7 .

\section{7. Энергии $\mathrm{E}_{k}$ и $\mathrm{T}_{k}$}

В разделе 6 мы видели, что теорему Балога-Семереди-Гауэрса можно рассматривать как результат о структуре множеств $A$, имеющих экстремальное соотношение между энергиями $\mathrm{E}_{1}(A)$ и $\mathrm{E}(A)$. Следующим шагом является изучение критических неравенств между другими типами энергий.

Первые результаты, где были рассмотрены иные пары энергий $\mathrm{E}_{k}, \mathrm{E}_{l}$, находящихся в экстремальном соотношении, были получены в [21]. В этой работе авторы имели дело с ситуацией, когда $k=2$, a $l>3$. Впоследствии все эти результаты были перекрыты теоремой из [22], к формулировке которой мы сейчас переходим.

Теорема 23. Пусть $K \geqslant 1$ - действительное число, $A \subseteq \mathbf{G}$ - некоторое множество, $\mathrm{E}(A)=|A|^{3} / K u \mathrm{E}_{3}(A)=M|A|^{4} / K^{2}$. Тогда найдется множество $A^{\prime} \subseteq A$ maкое, ито

$$
\left|A^{\prime}\right| \gg M^{-10} \log ^{-15} M \cdot|A|
$$

$u$

$$
\left|n A^{\prime}-m A^{\prime}\right| \ll\left(M^{9} \log ^{14} M\right)^{6(n+m)} K\left|A^{\prime}\right|
$$

для всех $n, m \in \mathbb{N}$. Более того, если $s \in(1,3)$ - действительное число и $\mathrm{E}_{s}(A)=|A|^{s+1} / K^{s-1}$, то для всех $s \in(1,3 / 2]$ существует множество $A^{\prime} \subseteq A$ со свойствами

$$
\left|A^{\prime}\right| \gg M^{-(14-4 s) /(3-s)}(s-1)^{21} \log ^{-21}\left(M(s-1)^{-1}\right) \cdot|A|
$$


u

$$
\left|n A^{\prime}-m A^{\prime}\right| \ll\left(M^{5}(s-1)^{-20} \log ^{20}\left(M(s-1)^{-1}\right)\right)^{6(n+m)} K\left|A^{\prime}\right| .
$$

Наконеи, если $s \in[3 / 2,3)$, то найдется множество $A^{\prime} \subseteq A$ такое, что

$$
\left|A^{\prime}\right| \gg M^{-(44-24 s) /(3-s)}(3-s)^{21} \log ^{-21} M \cdot|A|
$$

$u$

$$
\left|n A^{\prime}-m A^{\prime}\right| \ll\left(M^{(45-25 s) /(3-s)}(3-s)^{-20} \log ^{20} M\right)^{6(n+m)} K\left|A^{\prime}\right|
$$

для всех $n, m \in \mathbb{N}$.

Обсудим формулировку теоремы 23. В этой теореме есть два параметра - $K$ и $M$. На число $K$ нет никаких ограничений, и формулу $\mathrm{E}(A)=|A|^{3} / K$ можно считать его определением. Хотя на параметр $M$ формально тоже нет ограничений, тем не менее оценки (54), (55) и др. имеют смысл, только если число $M$ мало. Таким образом, параметры $K$ и $M$ неравноправны. Далее, рассмотрим для простоты лишь первую часть теоремы 23 и положим в неравенстве (55) $n=m=1$. Тогда $\left|A^{\prime}-A^{\prime}\right| \ll_{M} K\left|A^{\prime}\right|$. Кроме того, в силу оценки (54) имеем $\left|A^{\prime}\right| \gg_{M}|A|$. Применяя неравенство (13) и условие $\mathrm{E}(A)=|A|^{3} / K$, мы видим, что $\left|A^{\prime}-A^{\prime}\right| \gg_{M} K\left|A^{\prime}\right|$, и, значит, в терминах величины $M$ в случае $n=m=1$ оценка (55) является точной. Если же рассматривать бо́льшие $n$ и $m$, то данное неравенство говорит нам о том, что добавление или вычитание $A^{\prime}$ из множества $A^{\prime}-A^{\prime}$ не приводит к его увеличению (в терминах $K$ ). Иными словами, теорема 23 утверждает, что критическое соотношение между энергиями $\mathrm{E}_{2}(A)$ и $\mathrm{E}_{3}(A)$ означает, что множество $A$ обладает жесткой структурой, а именно, у него найдется подмножество $A^{\prime}$ такое, что $\left|A^{\prime}\right| \sim_{M}|A|,\left|A^{\prime}-A^{\prime}\right| \sim_{M}|A|^{4} \mathrm{E}^{-1}(A)$ и, наконец, его разностное множество $A^{\prime}-A^{\prime}$ является множеством с малым удвоением (как мы уже говорили выше, само множество $A^{\prime}$ не будет множеством с малым удвоением). В группе $\mathbb{F}_{2}^{n}$ можно сказать то же самое немного по-другому: если мы рассмотрим последовательность $A^{\prime}, 2 A^{\prime}, 3 A^{\prime}, \ldots$, то после второго сложения рост мощности множеств в ней прекратится (опять же в терминах $K)$.

Применения теоремы Балога-Семереди-Гауэрса обсуждались в разделе 6 . У теоремы 23 также имеется ряд приложений (см., например, [22], [117]-[119]). Мы обсудим самое простое из них. В работе [120] Хиф-Браун и Конягин (см. также [121]) доказали такой результат.

Теорема 24. Пусть $p$ - простое число $и$ $Г \subseteq \mathbb{F}_{p}^{*}-$ произвольная мультипликативная подгруппа, $|\Gamma|<p^{2 / 3}$. Тогда

$$
\mathrm{E}(\Gamma) \ll|\Gamma|^{5 / 2} .
$$

Таким образом, теорема 24 дает нетривиальную оценку для аддитивной энергии подгруппы. Такого рода результаты требуются в задачах о распределении элементов мультипликативных подгрупп (см., например, [31]). В работе [118] оценка (60) в случае достаточно малой подгруппы Г была усилена следующим образом: $\mathrm{E}(\Gamma) \ll|\Gamma|^{5 / 2-c}$, где $c>0$ - некоторая абсолютная 
константа. Мы опишем лишь основную идею доказательства этого результата, конечно, в реальности потребовались более строгие и прямые рассуждения. Итак, предположим, что оценка (60) по порядку точная, иными словами, $\mathrm{E}(\Gamma) \gg|\Gamma|^{5 / 2}$. Применяя метод Степанова из [120], можно показать, что всегда выполнено неравенство $\mathrm{E}_{3}(\Gamma) \ll|\Gamma|^{3} \log |\Gamma|$. В терминах величины $M$ это означает справедливость оценки $M \ll \log |\Gamma|$, следовательно, величина $M$ мала и, значит, мы вправе применить теорему 23. По этой теореме у подгруппы Г найдется большое подмножество $\Gamma^{\prime}$ такое, что $\Gamma^{\prime}-\Gamma^{\prime}$ является множеством с малым удвоением и, более того, от последующих сложений или вычитаний с $\Gamma^{\prime}$ наше разностное множество практически не растет. Но это уже дает требуемое противоречие, поскольку, с другой стороны, комбинаторные рассуждения показывают, что подгруппы (и их большие подмножества) являются базисами малого порядка [114], а значит, не расти не могут. Отсюда вытекает, что наше изначальное предположение $\mathrm{E}(\Gamma) \gg|\Gamma|^{5 / 2}$ было неверно, и, следовательно, $\mathrm{E}(\Gamma) \ll|\Gamma|^{5 / 2-c}, c>0$. Еще раз оговоримся, что эти рассуждения дают лишь грубую схему доказательства.

Наверное, самое интересное теоретико-числовое приложение метода доказательства теоремы 23 - это новая оценка для тригонометрической суммы Хейльбронна [119], которая связана с распределением частных Ферма $q(n)=$ $\left(n^{p-1}-1\right) / p, n \neq 0$ (см. [122]). Все ссылки, посвященные истории вопроса, а также приложениям частных Ферма, могут быть найдены, например, в статьях [120], [122], [123] (см. также [117]).

Теорема 25. Пусть $p$ - простое число и а-ненулевой вычет. Тогда

$$
\left|\sum_{n=1}^{p} \exp \left(2 \pi i \cdot \frac{a n^{p}}{p^{2}}\right)\right| \ll p^{5 / 6} \log ^{1 / 6} p .
$$

В разделе 6 мы видели, что теорема Балога-Семереди-Гауэрса является критерием для множества иметь подмножество с малым удвоением. Является ли критерием теорема 23? Легко видеть, что нет. Действительно, пусть $A=A_{1} \sqcup H \subseteq \mathbb{F}_{2}^{n}$, где $|H| \sim|A| / K^{1 / 3}$ - подпространство и множество $A_{1}-$ случайное. Тогда $\mathrm{E}(A) \sim|A|^{3} / K, \mathrm{E}_{3}(A) \geqslant \mathrm{E}_{3}(H)=|H|^{4} \gg|A|^{4} / K^{4 / 3}$ и у $A$ существует подмножество $A^{\prime}$, удовлетворяющее всем заключениям теоремы. Этим множеством будет, конечно, множество $A_{1}$.

С другой стороны, применяя неравенство Коши-Буняковского, легко видеть, что из заключений теоремы 23 вытекает оценка $\mathrm{T}_{k}(A) \gg_{M}|A|^{2 k-4} \mathrm{E}(A), k \geqslant 3$. Оказывается, что это более широкое соотношение уже является искомым критерием [23].

Теорема 26. Пусть $A \subseteq \mathbf{G}$ - произвольное множество и $M \geqslant 1$ - действительное число. Предположим, что

$$
\mathrm{T}_{4}(A) \geqslant \frac{|A|^{4} \mathrm{E}(A)}{M} .
$$

Тогда найдется множество $A^{\prime} \subseteq A$ такое, что

$$
\left|A^{\prime}\right| \gg \frac{|A|}{M^{3} \log ^{16 / 3}|A|}
$$


$u$

$$
\left|n A^{\prime}-m A^{\prime}\right| \ll\left(M^{3} \log ^{4}|A|\right)^{4(n+m)} M\left|A^{\prime}\right| \frac{|A|^{3}}{\mathrm{E}(A)},
$$

где $n, m \in \mathbb{N}$ - произвольные.

Более того, если

$$
\mathrm{T}_{2 s}(A) \geqslant \frac{|A|^{2 s} \mathrm{~T}_{s}(A)}{M},
$$

$s \geqslant 2$, то формулы выше остаются в силе. Наоборот, из оценок (62), (63) вытекает, что $\mathrm{T}_{2 s}(A) \gg_{M, \log |A|, s}|A|^{2 s} \mathrm{~T}_{s}(A)$.

В работе [20] (см. также предшествующую версию в статье [19]) была доказана новая структурная теорема о множествах с критическим соотношением между энергиями $\mathrm{T}_{4}(A)$ и $\mathrm{E}(A)$. Этот результат позволил Бэйтману и Кацу получить существенное продвижение в задаче о множествах без арифметических прогрессий в группе $\mathbb{F}_{3}^{n}$ (см. [19]).

Теорема 27. Пусть $\tau_{0}>0, \sigma_{0} \geqslant 0$ - действительные числа, $A \subseteq \mathbf{G}-$ произвольное множество, $A=-A$, обладающее свойством, что для всякого $A_{*} \subseteq A,\left|A_{*}\right| \gg|A|$, выполнено $\mathrm{E}\left(A_{*}\right) \gg \mathrm{E}(A)=|A|^{2+\tau_{0}}$. Предположим, что $\mathrm{T}_{4}(A) \ll|A|^{4+3 \tau_{0}+\sigma_{0}}$. Тогда существуют функиия $f_{\tau_{0}}:(0,1) \rightarrow(0, \infty)$ со свойством $f_{\tau_{0}}(\eta) \rightarrow 0, \eta \rightarrow 0$, и число $0 \leqslant \alpha \leqslant\left(1-\tau_{0}\right) / 2$ такие, что найдутся множества $X_{j}, H_{j} \subseteq \mathbf{G} u B_{j} \subseteq A, j \in\left[|A|^{\alpha-f_{\tau_{0}}\left(\sigma_{0}\right)}\right]$, для которых выполнены неравенства

$$
\begin{gathered}
\left|H_{j}\right| \ll|A|^{\tau_{0}+\alpha+f_{\tau_{0}}\left(\sigma_{0}\right)}, \quad\left|X_{j}\right| \ll|A|^{1-\tau_{0}-2 \alpha+f_{\tau_{0}}\left(\sigma_{0}\right)}, \\
\left|H_{j}-H_{j}\right| \ll\left|H_{j}\right|^{1+f_{\tau_{0}}\left(\sigma_{0}\right)}, \\
\left|\left(X_{j}+H_{j}\right) \cap B_{j}\right| \gg|A|^{1-\alpha-f_{\tau_{0}}\left(\sigma_{0}\right)}
\end{gathered}
$$

$u B_{i} \cap B_{j}=\varnothing$, если $i \neq j$.

Можно проверить, что любое множество $A$ с $\mathrm{E}(A)=|A|^{2+\tau_{0}}$, для которого существуют $X_{j}, H_{j}$, удовлетворяющие (65)-(67), является примером множества со свойством $\mathrm{T}_{4}(A) \ll|A|^{4+3 \tau_{0}+\sigma_{0}}$. Заметим также, что если $\mathrm{E}(A)=|A|^{2+\tau_{0}}$, то по неравенству Гёльдера выполнено $\mathrm{T}_{4}(A) \geqslant|A|^{4+3 \tau_{0}}$. Таким образом, теорема 27 дает нам полное описание множеств, имеющих критическое соотношение между парой энергий $\mathrm{E}(A)$ и $\mathrm{T}_{4}(A)$, т. е. она является критерием в нашем смысле.

Условие теоремы 27 " $A_{*} \subseteq A,\left|A_{*}\right| \gg|A|$, влечет $\mathrm{E}\left(A_{*}\right) \gg \mathrm{E}(A)=|A|^{2+\tau_{0}}$ " называется связностъю; оно может быть определено аналогичным образом для любой энергии, а не только аддитивной энергии E (см., например, [23]).

У теоремы 27 есть два крайних случая: $\alpha=0$ и $\alpha=\left(1-\tau_{0}\right) / 2$. Для простоты рассмотрим ситуацию, когда $\mathbf{G}=\mathbb{F}_{2}^{n}$. В случае $\alpha=0$ теорема Бэйтмана-Каца говорит, что наше множество $A$ является близким к множеству вида $H \dot{+} \Lambda$, где $H$ - подпространство, $|\Lambda| \sim|A| /|H| \sim|A|^{1-\tau_{0}}-$ базис, т. е. оно близко к множеству из семейства примера 5 (более точная формулировка изложена ниже, см. теорему 28). Возникает естественный вопрос: существует ли критерий для множества быть близким к множеству вида $H+\Lambda$ в терминах каких-либо 
энергий? Ответ здесь оказывается положительным - нужной парой энергий снова оказываются $\mathrm{E}(A)$ и $\mathrm{E}_{3}(A)$, но критическое соотношение между ними понимается теперь в другом смысле [23]. Легко видеть, что всегда выполнено неравенство $\mathrm{E}_{3}(A) \leqslant|A| \mathrm{E}(A)$, поэтому можно сказать, что $\mathrm{E}, \mathrm{E}_{3}$ находятся в критическом соотношении, если $\mathrm{E}_{3}(A) \gg|A| \mathrm{E}(A)$.

Теорема 28. Пусть $A \subseteq \mathbf{G}$ - произвольное множество $и M \geqslant 1, \varepsilon \in$ $(0,1)$ - действительные числа. Положим $K:=|A|^{3} / \mathrm{E}(A)$. В этом случае

$$
\mathrm{E}_{3}(A) \gg_{M, K^{\varepsilon}}|A| \mathrm{E}(A)
$$

тогда и толъко тогда, когда

$$
A \approx_{M, K^{\varepsilon}} \Lambda \dot{+} H,
$$

m.е. найдутся множества $H \subseteq \mathbf{G}, \Lambda \subseteq \mathbf{G}$ и элемент $z \in \mathbf{G}$ такие, что

$$
\begin{gathered}
|(H+z) \cap A| \gg_{M, K^{\varepsilon}} \frac{\mathrm{E}(A)}{|A|^{2}}, \quad|\Lambda| \ll_{M, K^{\varepsilon}} \frac{|A|}{|H|}, \\
|H-H| \ll_{M, K^{\varepsilon}}|H|
\end{gathered}
$$

$u$

$$
|A \cap(H+\Lambda)| \gg_{M, K^{\varepsilon}}|A| .
$$

Теорема 28 имеет интересное следствие о строении сумм/разностей множеств с критическим соотношением на пересечение их сдвигов [23]. Пусть множество $A \subseteq \mathbf{G}$ - произвольное, а $S=A+A$ и $D=A-A$ - его сумма и разность соответственно. Тогда, как легко видеть, для любого натурального $k$ и произвольных элементов $a_{1}, \ldots, a_{k} \in A$ выполнены включения

$$
A \subseteq\left(D+a_{1}\right) \cap\left(D+a_{2}\right) \cap \cdots \cap\left(D+a_{k}\right), \quad A \subseteq\left(S-a_{1}\right) \cap\left(S-a_{2}\right) \cap \cdots \cap\left(S-a_{k}\right) .
$$

Отсюда вытекает, в частности, что у множеств $S$ и $D$ всегда есть такие $\mathbf{x}=$ $\left(x_{1}, \ldots, x_{k}\right)$ с попарно различными компонентами $x_{j}$, что $\left|S_{\mathbf{x}}\right|,\left|D_{\mathbf{x}}\right| \geqslant|A|$. Оказывается, что если справедливо обратное неравенство, то структура множества $A$ обязана быть весьма жесткой.

Tеорема 29. Пусть $A \subseteq \mathbf{G}-$ произвольное множество, $D=A-A, S=$ $A+A, k \geqslant 2 u M \geqslant 1, \varepsilon \in(0,1)$ - действительные числа. Пусть такосе $K=|A|^{k+1} \mathrm{E}_{k}^{-1}(A)$. Предположим, что для всякого вектора $\mathbf{x}=\left(x_{1}, \ldots, x_{k-1}\right)$ с попарно различными компонентами $x_{j}, j \in[k]$, выполнено неравенство

$$
\left|D_{\mathbf{x}}\right| \leqslant M|A| \quad \text { или, аналогично, }\left|S_{\mathbf{x}}\right| \leqslant M|A| .
$$

Тогда либо $\mathrm{E}_{k}(A) \ll_{M,|A|^{\varepsilon}, k}|A|^{k}$, либо $\mathrm{E}(A) \gg_{M,|A|^{\varepsilon}, k}|A|^{3}$.

Итак, теорема 29 говорит, что условие (72) влечет две возможности для $A$ : оно близко либо к случайному множеству, либо, наоборот, к очень структурированному. Ясно, что оба варианта из теоремы 29 реализуются: первый случай когда $A$ действительно есть случайное множество, а второй - когда, например, наше $A$ - это множество с малым удвоением. 
В другом крайнем случае теоремы 27 , а именно когда $\alpha=\left(1-\tau_{0}\right) / 2$ (для простоты мы снова рассматриваем ситуацию $\left.\mathbf{G}=\mathbb{F}_{2}^{n}\right)$, наше множество $A$ выглядит как дизъюнктное объединение "аддитивно независимых" подпространств $H_{1}, \ldots, H_{k}$, где $k=|A|^{\left(1+\tau_{0}\right) / 2}$ (см. пример 7 в разделе 3$)$. Снова возникает вопрос: существует ли критерий в терминах энергий для множества быть близким к множеству такого типа? Ответ здесь снова оказывается положительным [23]. Чтобы сформулировать соответствующий результат, нам понадобится еще один тип энергий. (Ненормированной) нормой Гауэрса множества $A \subseteq \mathbf{G}$ называется выражение

$$
\|A\|_{\mathscr{U}^{d}}=\sum_{s_{1}, \ldots, s_{d}}\left|A_{\pi\left(s_{1}, \ldots, s_{d}\right)}\right|,
$$

где $\pi\left(s_{1}, \ldots, s_{d}\right)$ - вектор с $2^{d}$ компонентами, равными

$$
\left(\sum_{j=1}^{d} s_{j} \varepsilon_{j}\right), \quad \varepsilon_{j} \in\{0,1\}^{d} .
$$

Подробнее о нормах Гауэрса будет рассказано в разделе 8. Заметим, что

$$
\|A\|_{\mathscr{U}^{1}}=\mathrm{E}_{1}(A)=|A|^{2}, \quad\|A\|_{\mathscr{U}^{2}}=\mathrm{E}(A) \quad \text { и } \quad\|A\|_{\mathscr{U}^{3}}=\sum_{s} \mathrm{E}\left(A_{s}\right) .
$$

Очевидно, что

$$
\|A\|_{\mathscr{U}^{3}}=\sum_{s} \mathrm{E}\left(A_{s}\right) \leqslant \sum_{s}\left|A_{s}\right|^{3}=\mathrm{E}_{3}(A),
$$

и, следовательно, по неравенству Коши-Буняковского

$$
\|A\|_{\mathscr{U}^{3}}^{2} \leqslant \mathrm{E}_{4}(A) \mathrm{E}(A) .
$$

Критическое соотношение между $\|A\|_{\mathscr{\ell}^{3}}^{2}, \mathrm{E}_{4}(A)$ и $\mathrm{E}(A)$ состоит в обращении неравенства (73). Именно в этом случае множество $A$ оказывается близким к самодуальному, что и соответствует случаю $\alpha=\left(1-\tau_{0}\right) / 2$ теоремы 27.

Теорема 30. Пусть $A \subseteq \mathbf{G}$ - произвольное множество и $M \geqslant 1$ - действительное число. Пусть также $l=\log |A|$. Предположим, что множество А является $\mathscr{U}^{3}-u$ Е-связным. В этом случае неравенство

$$
\|A\|_{\mathscr{U}^{3}}^{2} \gg_{M} \mathrm{E}_{4}(A) \mathrm{E}(A)
$$

имеет место тогда и только тогда, когда найдутся действительное число $\Delta \sim_{M, l} \mathrm{E}_{3}(A) \mathrm{E}(A)^{-1}$ и множество

$$
P \subseteq\left\{s \in A-A: \Delta<\left|A_{s}\right|\right\}
$$

maкие, что $|P| \gg_{M, l}|A|, P=-P$,

$$
\mathrm{E}^{P}(A) \gg_{M, l} \mathrm{E}(A), \quad \mathrm{E}_{3}^{P}(A) \gg_{M, l} \mathrm{E}_{3}(A), \quad \mathrm{E}_{4}^{P}(A) \gg_{M, l} \mathrm{E}_{4}(A)
$$


u для всякого $s \in P$ существует множество $H^{s} \subseteq A_{s},\left|H^{s}\right| \gg_{M, l} \Delta$, со свойствами

$$
\left|H^{s}-H^{s}\right| \ll_{M, l}\left|H^{s}\right|
$$

$u \mathrm{E}\left(A, H^{s}\right) \ll_{M, l}\left|H^{s}\right|^{3}$.

Более того, найдутся непересекающиеся множества $H_{j} \subseteq A_{s_{j}},\left|H_{j}\right| \gg_{M, l} \Delta$, $s_{j} \in P, j \in[k]$, имеющие малое удвоение $(76)$, такие, что $\mathrm{E}\left(A, H_{j}\right) \ll_{M, l}\left|H_{j}\right|^{3}$ $u k \gg_{M, l}|A| \Delta^{-1}$.

Итак, мы видим, что экстремальные соотношения между разными типами энергий влекут за собой наличие жесткой структуры рассматриваемого множества, причем для описания этой структуры нам уже совершенно недостаточно одной константы удвоения, как это было в разделе 4. Наоборот, здесь требуется весь арсенал множеств из семейств раздела 3.

Интересно описать оставшиеся случаи теоремы 27 в терминах каких-либо энергий множеств.

\section{8. Структурные теоремы для норм Гауэрса}

В настоящем разделе мы обсудим одно важное понятие аддитивной комбинаторики и теории чисел, а именно нормы Гауэрса [8], [9], в свете структурных или, как говорят, обратных теорем для этих норм. Нормы Гауэрса нашли применения в теории чисел [8]-[15], [55], [56], динамических системах [124]-[130], а также в вопросах теоретической информатики [16], [17].

В этом разделе мы будем считать для простоты, что группа $\mathbf{G}$ конечна, $|\mathbf{G}|=N$. Пусть $d \geqslant 0$ - целое число и $\{0,1\}^{d}=\left\{\omega=\left(\omega_{1}, \ldots, \omega_{d}\right): \omega_{j} \in\right.$ $\{0,1\}, j=1,2, \ldots, d\}$ - обычный $d$-мерный куб. Для $\omega \in\{0,1\}^{d}$ пусть $|\omega|$ равно $\omega_{1}+\cdots+\omega_{d}$. Если $h=\left(h_{1}, \ldots, h_{d}\right) \in \mathbf{G}^{d}$, то $\omega \cdot h:=\omega_{1} h_{1}+\cdots+\omega_{d} h_{d}$. Пусть также $\mathscr{C}$ - это оператор комплексного сопряжения. Если $n$ - натуральное число, то $\mathscr{C}^{n}$ означает применение оператора комплексного сопряжения $n$ раз.

ОПРедЕлЕниЕ 1. Пусть $f: \mathbf{G} \rightarrow \mathbb{C}$ - произвольная функция. Равномерная норма Гауэрса (или просто норма Гауэрса) этой функции задается формулой

$$
\|f\|_{U^{d}}:=\left(\frac{1}{N^{d+1}} \sum_{x \in \mathbf{G}, h \in \mathbf{G}^{d}} \prod_{\omega \in\{0,1\}^{d}} \mathscr{C}^{|\omega|} f_{\omega}(x+\omega \cdot h)\right)^{1 / 2^{d}} .
$$

Можно показать, что выражение (77) действительно задает норму для всех $d \geqslant 2$ (см. [9] или [4]). В частности, справедливо неравенство треугольника

$$
\|f+g\|_{U^{d}} \leqslant\|f\|_{U^{d}}+\|g\|_{U^{d}}
$$

Кроме того, нормы Гауэрса, как и $L_{p}$-нормы, удовлетворяют неравенству монотонности

$$
\|f\|_{U^{d-1}} \leqslant\|f\|_{U^{d}}
$$

для всех $d \geqslant 2$. 
Из определения (77) вытекает, что для любой функции $f: \mathbf{G} \rightarrow \mathbb{C}$ такой, что $\|f\|_{\infty} \leqslant 1$, выполнено неравенство $\|f\|_{U^{d}} \leqslant 1$. Сформулируем основной вопрос этого раздела. Пусть $\delta \in(0,1]$ - действительное число и $f: \mathbf{G} \rightarrow \mathbb{C}-$ произвольная функция, $\|f\|_{\infty} \leqslant 1$, такая, что

$$
\|f\|_{U^{d}} \geqslant \delta \text {. }
$$

Есть ли у функции $f$ какая-то структура? Например, верно ли, что $f$ коррелирует с некоторой функцией из "хорошего" семейства?

Чтобы разобраться с постановкой вопроса, разберем сначала простейший случай $d=2$. Пользуясь формулой обращения (8), легко видеть, что $U^{2}$-норма функции $f$ выражается в терминах преобразования Фурье:

$$
\|f\|_{U^{2}}=\left(\frac{1}{N^{4}} \sum_{r \in \mathbf{G}}|\widehat{f}(r)|^{4}\right)^{1 / 4} .
$$

Таким образом, $U^{2}$-норма некоторой функции - это просто $L_{4}$-норма ее преобразования Фурье, умноженная на $N^{-1}$. Из формулы (81) вытекает такое утверждение.

Лемма 10. Пусть $f: \mathbf{G} \rightarrow \mathbb{C}-$ произвольная функиия $c\|f\|_{\infty} \leqslant 1$. Тогда $\|\widehat{f}\|_{\infty} \leqslant\|\widehat{f}\|_{4}$. Наоборот, $\|f\|_{U^{2}}=N^{-1}\|\widehat{f}\|_{4} \leqslant\|\widehat{f}\|_{\infty}^{1 / 2} N^{1 / 2}$.

Первое неравенство леммы очевидно, а второе вытекает из равенства Парсеваля:

$$
\|\widehat{f}\|_{4}^{4}=\sum_{r}|\widehat{f}(r)|^{4} \leqslant\|\widehat{f}\|_{\infty}^{2}\|\widehat{f}\|_{2}^{2}=N\|\widehat{f}\|_{\infty}^{2}\|f\|_{2}^{2} \leqslant\|\widehat{f}\|_{\infty}^{2} N^{2} .
$$

Таким образом, если неравенство (80) справедливо для $d=2$, то для функции $f$ найдется $\xi \in \widehat{\mathbf{G}}$ такое, что

$$
|\widehat{f}(\xi)|=\left|\sum_{x} f(x) \overline{\xi(x)}\right| \geqslant \delta^{C} N,
$$

где $C>0$ - некоторая абсолютная константа. Наоборот, оценка (82) автоматически означает, что у функции $f$ большая $U^{2}$-норма. Значит, в ситуации, когда $d=2$, неравенство (80) возможно тогда и только тогда, когда функция $f$ коррелирует с некоторым характером.

Что можно сказать в случае старших норм? Хорошо известно, что даже для $d=3$ норма Гауэрса не выражается через коэффициенты Фурье функции и, следовательно, аналог формулы (81) не имеет места. Тем не менее ответить на основной вопрос этого раздела, сформулированный после $(80)$, можно и в этой, более сложной, ситуации. Первым результатом, обобщающим неравенство (82) для $d>2$, была следующая теорема Гауэрса [9] (случай $\mathbf{G}=\mathbb{Z}_{N}$ ).

Теорема 31. Пусть $\delta>0$ - действительное число, $f: \mathbb{Z}_{N} \rightarrow \mathbb{C}$ - некоторая функиия, $\|f\|_{\infty} \leqslant 1, \sum_{x} f(x)=0 u\|f\|_{U^{d}} \geqslant \delta$. Тогда найдутся абсолютные константы $C_{d}, c_{d}>0$ такие, что для всех $N \geqslant \exp \left\{C_{d} \delta^{-C_{d}}\right\}$ существуют многочлен $p_{d-1}(x)$ степени $d-1$ и арифметическая прогрессия $P$, 
$|P| \gg c_{d} \delta^{C_{d}} N^{c_{d} \delta^{C_{d}}}$, для которых выполнено неравенство

$$
\left|\sum_{x \in P} f(x) e\left(p_{d-1}(x)\right)\right| \geqslant c_{d} \delta^{C_{d}}|P| .
$$

В очень грубом смысле доказательство теоремы 31 похоже на метод получения теоремы 21 из раздела 6. Гауэрс показывает, что условие (80) эквивалентно тому, что некоторые производные функции $f$ (так называемые "разностные функции") имеют, в некотором роде, большую аддитивную энергию, а затем применяет теорему Балога-Семереди-Гауэрса для получения из этого факта чисто комбинаторных следствий (более подробно см. статьи [8], [9] и обзоры [4], [33]). Интересно, что для доказательства своего результата в первой статье [8] Гауэрс соединял теорему Балога-Семереди-Гауэрса с теоремой Фреймана.

В ситуации, когда $d=3$, теорема 31 была перенесена Грином и Тао на случай произвольной абелевой группы $\mathbf{G}$ (см. [10]). Грубо говоря, в группе $\mathbf{G}$ арифметическая прогрессия $P$ в формуле (83) должна быть заменена на трансляцию множества Бора. Мы не хотим на этом подробно останавливаться, заметим только, что теорема 31 обладает по сравнению с оценкой (82) значительным недостатком. Этот недостаток состоит в том, что суммирование в формуле (83) идет лишь по прогрессии $P$, а не по всей группе, как в (82). Теоремы, обладающие тем же свойством локальности, что и теорема 31, называют слабыми обратными теоремами для норм Гауэрса, а если имеется глобальная корреляция как в формуле (82), то - сильными обратными теоремами. Известно, что именно последние и нужны для получения результатов об арифметических прогрессиях в простых числах (см. [57]), а также, например, для таких утверждений, как теоремы 5, 6 раздела 4.

Сначала сильная обратная теорема для $U^{3}$-нормы Гауэрса была доказана в случае конечных векторных пространств над простым полем (см. [10] для случая $p \neq 2$ и [18] для случая $p=2$ ).

Теорема 32. Пусть $\delta>0$ - действителъное число, $f: \mathbb{F}_{p}^{n} \rightarrow \mathbb{C}$-некоторая функиия, $\|f\|_{\infty} \leqslant 1 u\|f\|_{U^{3}} \geqslant \delta$. Тогда найдутся $(n \times n)$-матрица $M$, вектор $b \in \mathbb{F}_{p}^{n}$, а также константа $c(\delta)>0$ такие, что

$$
\left|\sum_{x} f(x) \exp \left(\frac{2 \pi i(\langle M x, x\rangle+b x)}{p}\right)\right| \geqslant c(\delta) p^{n} .
$$

Таким образом, в ситуации $\mathbf{G}=\mathbb{F}_{p}^{n}$ условие $\|f\|_{U^{3}} \geqslant \delta$ означает глобальную корреляцию с квадратичным тригонометрическим многочленом вида

$$
\exp \left(\frac{2 \pi i(\langle M x, x\rangle+b x)}{p}\right) \text {. }
$$

Несложно показать, что, обратно, из условия (84) вытекает, что $\|f\|_{U^{3}} \geqslant C(\delta)$, где $C(\delta)>0$ - некоторая константа, и, значит, теорема 32 полностью описывает все функции на $\mathbb{F}_{p}^{n}$ с большой $U^{3}$-нормой. 
$\mathrm{K}$ сожалению, даже в случае групп $\mathbb{F}_{p}^{n}$ для $d>3$ аналог теоремы 32 уже не имеет места. Если же мы рассматриваем общие абелевы группы, то ситуация становится еще сложнее. Окончательный ответ был дан в [12], [13], где также может быть найдена и история вопроса. В эргодической постановке результат ниже был получен несколько ранее (см. [124], [130]).

Напоминаем, что нильмногообразием степени $d$ называется многообразие вида $G / \Gamma$, где $G$ - связная, односвязная нильпотентная группа Ли порядка $d$ (т. е. ее $(d+1)$-й коммутатор тривиален), а $\Gamma$ - решетка, т. е. дискретная кокомпактная подгруппа группы $G$.

Теорема 33. Пусть $d$ - натуральное число и $\delta \in(0,1]$ - действительное число. Тогда найдется конечное семейство $\mathscr{M}_{d, \delta}$ нильмногообразий $G / \Gamma$ степени $d$, снабженных гладкой римановой метрикой $D_{G / \Gamma}$, а также константы $C(d, \delta), c(d, \delta)>0$ со следующими свойствами. Для любого натурального $N$ и произвольной функици $f:[N] \rightarrow \mathbb{C},\|f\|_{\infty} \leqslant 1$, такой, что

$$
\|f\|_{U^{d+1}[N]} \geqslant \delta
$$

найдутся нильмногообразие $G / \Gamma \in \mathscr{M}_{d, \delta}$, некоторый элемент $g \in G$ и функиия $F: G / \Gamma \rightarrow \mathbb{C},\|F\|_{\infty} \leqslant 1$, с константой Липшица, относительно метрики $D_{G / \Gamma}$ не превосходящей $C(d, \delta)$, для которых выполнено неравенство

$$
\left|\sum_{n=1}^{N} f(n) \overline{F\left(g^{n} \Gamma\right)}\right| \geqslant c(d, \delta) N .
$$

Последовательность $F\left(g^{n} x\right)$ в (86) называется нильпоследовательностью; таким образом, теорема 33 утверждает, что любая функция с большой нормой Гауэрса (85) коррелирует с некоторой нильпоследовательностью. Замечательно, что обратное также верно (см. [10], [124]), причем доказательство этого факта сравнительно простое. Таким образом, теорема 33 является критерием для функции иметь большую норму Гауэрса. Подробнее о нильпоследовательностях можно прочитать, например, в работе [12], а также в [124].

Оценки величины $C(d, \delta), c(d, \delta)$ в статьях [12], [13] совершенно неконструктивны, кроме некоторых частных случаев, например $d=3$. Заметим, что в последней ситуации существует тесная связь с результатами и гипотезами раздела 4, в частности с полиномиальной гипотезой Фреймана-Ружи (см. [55]).

Из сложных теорем 32, 33 уже достаточно просто вытекают общие результаты о декомпозиции ограниченных функций. Мы приведем здесь лишь две декомпозиционные теоремы для групп $\mathbb{F}_{p}^{n}$. Первый совсем простой результат отвечает неравенству (82), а второй использует в своем доказательстве обратную теорему 32. В нашем изложении мы следуем лекциям [28].

Пусть $\varphi_{1}, \ldots, \varphi_{k}: \mathbb{F}_{p}^{n} \rightarrow \mathbb{F}_{p}$ - произвольные функции. Зададим $\sigma$-алгебру (разбиение) $\mathscr{S}$ группы $\mathbb{F}_{p}^{n}$ на атомы, состоящие из множества уровней

$$
\left\{x: \varphi_{1}(x)=c_{1}, \ldots, \varphi_{k}(x)=c_{k}\right\},
$$


где $c_{1}, \ldots, c_{k} \in \mathbb{F}_{p}$ - некоторые константы. Обычным образом определяется условное математическое ожидание $\mathbf{E}(f \mid \mathscr{S})$ функции $f: \mathbb{F}_{p}^{n} \rightarrow \mathbb{C}$. Число $k$ называется сложностъю $\sigma$-алгебры $\mathscr{S}$. Наконец, если все функции $\varphi_{j}-$ линейные, то назовем $\mathscr{S}$ линейным фактором. Справедлива следующая теорема [28].

Теорема 34. Пусть $f: \mathbb{F}_{p}^{n} \rightarrow \mathbb{C}-$ произвольная функиия, $\|f\|_{\infty} \leqslant 1, u$ пусть $\varepsilon>0$ - действительное число. Тогда найдется линейный фактор $\mathscr{S}$ сложности не более $4 \varepsilon^{-4}$ такой, что

$$
f=f_{1}+f_{2},
$$

где $f_{1}:=\mathbf{E}(f \mid \mathscr{S})$ u $\left\|f_{2}\right\|_{U^{2}} \leqslant \varepsilon$.

Доказательство. Пусть $S=\operatorname{Spec}_{\varepsilon^{2} / 2}(f):=\left\{\xi \in \mathbb{F}_{p}^{n}:|\widehat{f}(\xi)| \geqslant 2^{-1} \varepsilon^{2}\|f\|_{2}\right\}$. Из равенства Парсеваля (5) имеем $|S| \leqslant 4 \varepsilon^{-4}$. Пусть $S$ состоит из элементов $s_{1}, \ldots, s_{|S|}$ и $H=S^{\perp}$. Положим $f_{1}=f * \mu_{H}$ и $f_{2}=f-f * \mu_{H}$. Легко видеть, что $f_{1}=\mathbf{E}(f \mid \mathscr{S})$, где $\sigma$-алгебра $\mathscr{S}$ порождена функциями $\varphi_{j}(x)=\left\langle s_{j}, x\right\rangle, j \in[|S|]$. Далее, $\widehat{f}_{2}(\xi)=\widehat{f}(\xi)\left(1-\widehat{\mu}_{H}(\xi)\right)$ и, следовательно, $\widehat{f}_{2}(\xi)=0$ для $\xi \in S$. Отсюда $\left\|\widehat{f}_{2}\right\|_{\infty} \leqslant \varepsilon^{2}$. Наконец, из леммы 10 вытекает, что $\left\|f_{2}\right\|_{U^{2}} \leqslant \varepsilon$. Теорема доказана.

Итак, теорема 34 - это по сути простое разложение функции на две части, содержащие соответственно маленькие и большие коэффициенты Фурье. Ясно, что если требуется доказать аналог теоремы 34 для старших норм Гауэрса, то мы столкнемся со значительными сложностями, поскольку, как обсуждалось ранее, в этой ситуации не работают методы классического гармонического анализа. Тем не менее, используя обратные теоремы для $U^{d}$-норм, можно доказать подобные обобщения. Для простоты мы рассмотрим случай $\mathbf{G}=\mathbb{F}_{5}^{n}, d=3$.

Назовем $\mathscr{S}$ квадратичным фактором, если все функции $\varphi_{j}(x)$ имеют вид $\varphi_{j}(x)=\left\langle M_{j} x, x\right\rangle+\left\langle s_{j} x\right\rangle$, где $M_{j}$ - симметричные матрицы, а $s_{j}$ - произвольные векторы.

Теорема 35. Пусть $\varepsilon>0$ - действительное число $и \omega: \mathbb{R}^{+} \rightarrow \mathbb{R}^{+}$- произвольная возрастающая функиия. Пусть также $n \geqslant n_{0}(\varepsilon, \omega)-$ достаточно большое число и $f: \mathbb{F}_{5}^{n} \rightarrow \mathbb{C}-$ произвольная функиия, $\|f\|_{\infty} \leqslant 1$. Тогда найдется квадратичный фактор $\mathscr{S}$ сложности $d, d \leqslant M(\varepsilon, \omega)$, такой, что

$$
f=f_{1}+f_{2}+f_{3},
$$

где $f_{1}:=\mathbf{E}(f \mid \mathscr{S}),\left\|f_{2}\right\|_{2} \leqslant \varepsilon u\left\|f_{3}\right\|_{U^{3}} \leqslant 1 / \omega(d)$.

Конечно, доказательство теоремы 35 уже не столь просто, как доказательство теоремы о $U^{2}$-норме. Основная идея состоит в следующем (более подробно см. [28] и [4]). Если функция $f$ обладает малой $U^{3}$-нормой, то доказывать нечего, мы просто полагаем $f_{3}=f, f_{2}=0, \mathscr{S}=\{\varnothing, \mathbf{G}\}$. Если же $U^{3}$-норма $f$ велика, то по теореме 32 имеется корреляция с квадратичной формой. Рассматривая множества уровней этой формы, мы получаем $\sigma$-алгебру $\mathscr{S}_{1}$. Затем вычисляем $\mathbf{E}\left(f \mid \mathscr{S}_{1}\right)$ и применяем предыдущие рассуждения к функции $f-\mathbf{E}\left(f \mid \mathscr{S}_{1}\right)$. Если ее $U^{3}$-норма мала, то мы останавливаем нашу процедуру, 
полагая $f_{3}=f-\mathbf{E}\left(f \mid \mathscr{S}_{1}\right)$, а если нет, то, снова применяя теорему 32, находим еще одну квадратичную форму, используя ее, измельчаем $\sigma$-алгебру $\mathscr{S}_{1}$, получая $\sigma$-алгебру $\mathscr{S}_{2}$ и т.д. В итоге наш алгоритм сойдется и мы получим разложение (87).

Надо сказать, что разложения, подобные теоремам 34,35 , берут свое начало из работы [131] и леммы регулярности Семереди [132], [62].

Обратные теоремы и разложения выше обобщались на разные ситуации. В настоящем обзоре мы не будем останавливаться на этом вопросе, см., например, работы [12], [13], [128] и др. Скажем только, что именно замечательное разложение неограниченных функций специального класса (вроде модифицированной функции Мангольдта) из статьи [57] позволило Грину и Тао получить результат о простых числах в арифметических прогрессиях. Полезность для теории чисел подобных декомпозиций достаточно очевидна. Действительно, рассмотрим, для простоты, теорему 35 и дадим с ее помощью схему доказательства теоремы Семереди о прогрессиях длины четыре в группе $\mathbb{F}_{5}^{n}$ (применение теоремы 34 позволяет получить теорему Семереди о прогрессиях длины три, причем еще проще). Другими словами, нам надо показать, что любое множество $A \subseteq \mathbb{F}_{5}^{n}$ положительной плотности содержит прогрессию $\{x, x+d, x+2 d, x+3 d\}, d \neq 0$. Предположим противное, т. е. что некоторое множество $A \subseteq \mathbb{F}_{5}^{n},|A|=\delta \cdot 5^{n}, \delta \gg 1, n \rightarrow \infty$, не имеет конфигураций вида $\{x, x+d, x+2 d, x+3 d\}, d \neq 0$. Легко видеть, что число арифметических прогрессий длины четыре (включая вырожденный случай $d=0$ ) в множестве $A$ есть

$$
\Lambda_{4}(A):=\sum_{x, d} A(x) A(x+d) A(x+2 d) A(x+3 d)
$$

и оно равняется, по нашему предположению, $|A|=\delta \cdot 5^{n}=o\left(5^{2 n}\right)$. Рассмотрим балансовую функцию множества $A$, т. е. функцию $f(x)=A(x)-\delta$. Пользуясь полилинейностью функционала $\Lambda_{4}$, несложно проверить, что $\Lambda_{4}(A) \approx$ $\delta^{4} \cdot 5^{2 n}+\Lambda_{4}(f)$, и, значит, если показать, что $\Lambda_{4}(f)=o\left(5^{2 n}\right)$, то мы придем к противоречию. Применяя декомпозиционную теорему 35 к функции $f$, легко убедиться, что $\Lambda_{4}(f)$ выражается через $\Lambda_{4}\left(f_{j}\right), j=1,2,3$. Но число $\Lambda_{4}\left(f_{2}\right)$ может быть сделано сколь угодно малым посредством выбора параметра $\varepsilon$. Далее, величина $\Lambda_{4}\left(f_{3}\right)$ также мала, поскольку (и это причина, по которой Гауэрс ввел свои нормы) $U^{3}$-норма контролирует $\Lambda_{4}$. Значит, оставшаяся величина $\Lambda_{4}\left(f_{1}\right)$ обязана быть большой. Но функция $f_{1}$ постоянна на хорошо устроенной $\sigma$-алгебре $\mathscr{S}$ с малым числом атомов, которые, как можно показать, суть подпространства $\mathbb{F}_{5}^{n}$. С другой стороны, среднее балансовой функции равно нулю. Отсюда вытекает, что множество $A$ имеет строго бо́льшую, чем $\delta$, плотность на некотором подпространстве $\sigma$-алгебры $\mathscr{S}$. Это дает возможность проитерировать нашу процедуру, рассматривая лишь пересечение множества $A$ с этим подпространством. И так далее. В итоге, после нескольких применений описанного алгоритма, плотность $A$ в некотором подпространстве достигнет 1 и это приведет к противоречию с отсутствием у $A$ арифметических прогрессий длины четыре. Более подробно, см., например, обзор [4]. 


\section{9. Заключение}

Мы заканчиваем настоящий обзор формулировкой нескольких нерешенных проблем аддитивной комбинаторики. Часть проблем была уже сформулирована в предыдущих разделах, например, полиномиальная гипотеза Фреймана-Ружи, полиномиальная гипотеза Боголюбова (см. раздел 4), вопросы об обратных теоремах для норм Гауэрса (раздел 8) и т. д.

Важной, может быть даже центральной, задачей в аддитивной комбинаторике является вопрос об определении необходимых и достаточных условий для того, чтобы множество $S$ имело вид $A+A, A-A$, а также являлось старшей суммой или разностью. Иными словами, какие специфические свойства отделяют сумму или разность от произвольного множества? Это сложная проблема, не поддающаяся решению даже в случае хорошо изученных семейств (см. [133], [134]). Тем не менее в последнее время здесь наметилось небольшое продвижение [135], [23].

Еще одним сложным вопросом остается гипотеза Эрдёша-Турана [136].

ГиПотеЗА 6. Пусть $A=\left\{n_{1}<n_{2}<\cdots\right\}$ - бесконечная последовательность натуральных чисел такая, что

$$
\sum_{i=1}^{\infty} \frac{1}{n_{i}}=\infty .
$$

Тогда А содержит арифметическую прогрессию любой длины.

Помимо того, что из гипотезы 6 автоматически вытекает теорема Грина-Тао об арифметических прогрессиях [57], она интересна еще и потому, что постоянно дает мотивацию для развития все новых методов и даже целых областей аддитивной комбинаторики. Так, даже частичный прогресс в ее решении потребовал создания изящных комбинаторных подходов [61], [62], [66], [137], [138], тонких инструментов из анализа Фурье [50], [83], [104], [105], [131], [139], [140], структурных аддитивно-комбинаторных результатов [19], [20], эргодических идей [124]-[126], [141], [142], и др. Благодаря этой проблеме мы имеем новые мощные инструменты, такие как лемма регулярности Семереди [132] (см. гиперграфовую версию в [143]-[146]), принцип соответствия Фюрстенберга [141], глубокие результаты из теории больших тригонометрических сумм [50], [83], [104] и т. д. Более подробно см. обзоры [4], [33].

Простейший случай гипотезы 6 относительно мощности множеств без прогрессий длины три принимает более сильную форму в случае конечномерных векторных пространств над простым полем. Так, в работе [3] Грин высказал следующее предположение.

ГиПотезА 7. Пусть $A \subseteq \mathbb{F}_{3}^{n}$ - произвольное множество без решений уравнения $x+y+z=0$. Тогда найдется абсолютная константа $\delta>0$ такая, что $|A| \leqslant(3-\delta)^{n}$.

В случае группы $\mathbb{F}_{2}^{n}$ по теореме Конягина (24) мы знаем, что множество $2 A-2 A$ содержит подпространство малой коразмерности. Что можно сказать 
о подпространствах в множествах вида $A+A$, где $A \subseteq \mathbb{F}_{2}^{n}$ достаточно плотное, например, $|A| \gg 2^{n}$ ? Известно [3], [68], что коразмерность максимального подпространства $H$ из суммы может быть, по крайней мере, $\sqrt{n}$. С другой стороны, в настоящее время имеются лишь очень плохие нижние оценки на размерность $H$, а именно $\operatorname{dim} H \gg n$ (см. [3], [147]). Интересно получить хоть какое-то неравенство вида $\operatorname{codim}(H)=o(n)$ на коразмерность максимального подпространства $H$ в суммах.

Сам по себе интересен вопрос о строении множеств больших тригонометрических сумм. Каковы необходимые и достаточные условия для того, чтобы некоторое множество было множеством вида $\operatorname{Spec}_{c}$ ? Неизвестен ответ даже на более специальный вопрос: что можно сказать о структуре множеств $\mathrm{Spec}_{c}$, мощность которых близка к числу $1 /\left(\delta c^{2}\right)$ - верхней границе для мощности множества больших тригонометрических сумм, вытекающей из равенства Парсеваля? Подобные результаты могли бы помочь в решении задач комбинаторной теории чисел, использующих анализ Фурье.

До сих пор ни для одного линейного уравнения $c_{1} x_{1}+\cdots+c_{n} x_{n}=0$ при $n$, не зависящем от размера группы $\mathbf{G}$, не найдено точное значение его рамсеевского числа (см. книгу [148] и обзор [33]), т. е. минимального количества цветов, требуемых, чтобы раскрасить $\mathbf{G}$ и не получить ни одного одноцветного решения нашего уравнения.

Мы заканчиваем список проблем формулировкой главного вопроса теории сумм произведений [111] - гипотезой Эрдёша-Семереди. Некоторые задачи о суммах произведений уже обсуждались нами в разделе 6 .

ГиПотезА 8. Пусть $A \subset \mathbb{R}$ - произвольное множество. Тогда для любого $\varepsilon>0$ выполнено неравенство

$$
\max \{|A+A|,|A A|\} \gg|A|^{2-\varepsilon} .
$$

В настоящий момент гипотеза 8 остается недоказанной и для других колец, например для $\mathbb{Z}$ и $\mathbb{C}$. Аналог гипотезы Эрдёша-Семереди в конечных полях давал бы целый ряд важных приложений (см., например, [149], а также [106], [114], [115]).

Автор выражает благодарность Т. Шоену и К. С. Рютину за ряд полезных обсуждений и замечаний.

\section{Список литературы}

[1] M. B. Nathanson, Additive number theory. Inverse problems and the geometry of sumsets, Grad. Texts in Math., 165, Springer-Verlag, New York, 1996, xiv+293 pp.

[2] T. Tao, V. Vu, Additive combinatorics, Cambridge Stud. Adv. Math., 105, Cambridge Univ. Press, Cambridge, 2006, xviii+512 pp.

[3] B. Green, "Finite field models in additive combinatorics", Surveys in combinatorics, London Math. Soc. Lecture Note Ser., 327, Cambridge Univ. Press, Cambridge, 2005, 1-28. 
[4] И. Д. Шкредов, "Теорема Семереди и задачи об арифметических прогрессиях", УМН, 61:6(372) (2006), 111-178; англ. пер.: I. D. Shkredov, "Szemerédi's theorem and problems on arithmetic progressions", Russian Math. Surveys, 61:6 (2006), 1101-1166.

[5] B. Green, Approximate algebraic structure, 2014, 25 pp., arXiv: 1404.0093v1.

[6] W. T. Gowers, "Rough structure and classification", GAFA 2000 "Visions in mathematics" (Tel Aviv, 1999), Geom. Funct. Anal., 2000, Special volume, Part I, 79-117.

[7] Г. А. Фрейман, Начала структурной теории сложения множеств, Казан. гос. пед. ин-т, Казань, 1966, 140 с.

[8] W.T. Gowers, "A new proof of Szemerédi's theorem for arithmetic progressions of length four", Geom. Funct. Anal., 8:3 (1998), 529-551.

[9] W. T. Gowers, "A new proof of Szemerédi's theorem", Geom. Funct. Anal., 11:3 (2001), 465-588.

[10] B. Green, T. Tao, "An inverse theorem for the Gowers $U^{3}(G)$ norm", Proc. Edinb. Math. Soc. (2), 51:1 (2008), 73-153.

[11] B. Green, T. Tao, T. Ziegler, "An inverse theorem for the Gowers $U^{4}$-norm", Glasg. Math. J., 53:1 (2011), 1-50.

[12] B. Green, T. Tao, T. Ziegler, "An inverse theorem for the Gowers $U^{s+1}[N]$-norm", Electron. Res. Announc. Math. Sci., 18 (2011), 69-90.

[13] B. Green, T. Tao, T. Ziegler, "An inverse theorem for the Gowers $U^{s+1}[N]$-norm", Ann. of Math. (2), 176:2 (2012), 1231-1372.

[14] B. Green, T. Tao, "Linear equations in primes", Ann. of Math. (2), 171:3 (2010), 1753-1850; 2006 (v2 - 2008), 83 pp., arXiv: math/0606088v1.

[15] B. Green, "Generalising the Hardy-Littlewood method for primes", International congress of mathematicians, v. II, Eur. Math. Soc., Zürich, 2006, 373-399; 2006, 26 pp., arXiv: math/0601211v1.

[16] S. Lovett, R. Meshulam, A. Samorodnitsky, "Inverse conjecture for the Gowers norm is false", Theory Comput., 7 (2011), 131-145; 2007 (v3 - 2008), 20 pp., arXiv: $0711.3388 \mathrm{v} 1$.

[17] A. Samorodnitsky, L. Trevisan, "Gowers uniformity, influence of variables, and PCPs", SIAM J. Comput., 39:1 (2009), 323-360.

[18] A. Samorodnitsky, "Low-degree tests at large distances", STOC'07 - Proceedings of the 39th Annual ACM Symposium on Theory of Computing, ACM, New York, 2007, 506-515.

[19] M. Bateman, N.H. Katz, "New bounds on cap sets", J. Amer. Math. Soc., 25:2 (2012), 585-613.

[20] M. Bateman, N. H. Katz, Structure in additively nonsmoothing sets, 2011, 18 pp., arXiv: $1104.2862 \mathrm{v} 1$.

[21] T. Schoen, I. D. Shkredov, "Higher moments of convolutions", J. Number Theory, 133:5 (2013), 1693-1737.

[22] И. Д. Шкредов, "Несколько новых результатов о старших энергиях", Тр. ММО, 74, МЦНМО, М., 2013, 35-73; англ. пер.: I. D. Shkredov, "Some new results on higher energies", Trans. Moscow Math. Soc., 2013:1 (2013), 31-63.

[23] I. D. Shkredov, "Energies and structure of additive sets", Electron. J. Combin., 21:3 (2014), paper 3.44, 53 pp.; 2014, 52 pp., arXiv: 1405.3132v1. 
[24] E. Croot, O. Sisask, "A probabilistic technique for finding almost-periods of convolutions", Geom. Funct. Anal., 20:6 (2010), 1367-1396.

[25] E. Croot, I. Łaba, O. Sisask, "Arithmetic progressions in sumsets and $L^{p}$-almostperiodicity", Combin. Probab. Comput., 22:3 (2013), 351-365; 2011 (v2 - 2013), 13 pp., arXiv: 1103.6000v1.

[26] M.-C. Chang, "A polynomial bound in Freiman's theorem", Duke Math. J., 113:3 (2002), 399-419.

[27] T. Sanders, "The structure theory of set addition revisited", Bull. Amer. Math. Soc. (N.S.), 50:1 (2013), 93-127.

[28] B. Green, "Montréal notes on quadratic Fourier analysis", Additive combinatorics, CRM Proc. Lecture Notes, 43, Amer. Math. Soc., Providence, RI, 2007, 69-102; 2006 (v2 - 2007), 34 pp., arXiv: math.CA/0604089v1.

[29] W. Rudin, Fourier analysis on groups, Reprint of the 1962 original, Wiley Classics Lib., New York, 1990, x+285 pp.

[30] И.М. Виноградов, Основы теории чисел, 10-е изд., стер., Лань, СПб., 2004, 176 с.; англ. пер. 5-го изд.: I. M. Vinogradov, Elements of number theory, Dover Publications, Inc., New York, 1954, viii+227 pp.

[31] S. V. Konyagin, I. E. Shparlinski, Character sums with exponential functions and their applications, Cambridge Tracts in Math., 136, Cambridge Univ. Press, Cambridge, 1999, viii+163 pp.

[32] N. Alon, J. Spencer, The probabilistic method, Wiley-Intersci. Ser. Discrete Math. Optim., John Wiley \& Sons, Inc., New York, 1992, xvi+254 pp.

[33] И. Д. Шкредов, “Анализ Фурье в комбинаторной теории чисел”, УМН, 65:3(393) (2010), 127-184; англ. пер.: I. D. Shkredov, "Fourier analysis in combinatorial number theory", Russian Math. Surveys, 65:3 (2010), 513-567.

[34] А. А. Карацуба, Основы аналитической теории чисел, 2-е изд., Наука, М., 1983, 240 с.; англ. пер.: А. A. Karatsuba, Basic analytic number theory, Springer-Verlag, Berlin, 1993, xiv+222 pp.

[35] Н. М. Коробов, Тригонометрические суммы и их приложения, Наука, М., 1989, 240 с.; англ. пер.: N. M. Korobov, Exponential sums and their applications, Math. Appl. (Soviet Ser.), 80, Kluwer Academic Publishers Group, Dordrecht, 1992, xvi+209 pp.

[36] T. Schoen, "Near optimal bounds in Freiman's theorem", Duke Math. J., 158:1 (2011), 1-12.

[37] T. Sanders, "On the Bogolyubov-Ruzsa lemma", Anal. PDE, 5:3 (2012), 627-655.

[38] M. Kneser, "Abschätzung der asymptotischen Dichte von Summenmengen", Math. Z., 58:1 (1953), 459-484.

[39] B. Green, I. Z. Ruzsa, "Freiman's theorem in an arbitrary abelian group", J. Lond. Math. Soc. (2), 75:1 (2007), 163-175.

[40] T. Tao, "Product set estimates for non-commutative groups", Combinatorica, 28:5 (2008), 547-594.

[41] H. A. Helfgott, "Growth and generation in $\mathrm{SL}_{2}(\mathbb{Z} / p \mathbb{Z})$ ", Ann. of Math. (2), 167:2 (2008), 601-623.

[42] H. A. Helfgott, "Growth in $\mathrm{SL}_{3}(\mathbb{Z} / p \mathbb{Z})$ ", J. Eur. Math. Soc. (JEMS), 13:3 (2011), 761-851. 
[43] E. Breuillard, B. Green, T. Tao, "The structure of approximate groups", Publ. Math. Inst. Hautes Études Sci., 116:1 (2012), 115-221.

[44] E. Hrushovski, "Stable group theory and approximate subgroups", J. Amer. Math. Soc., 25:1 (2012), 189-243; 2009 (v4 - 2011), 32 pp., arXiv: 0909.2190v1.

[45] M. Gromov, "Groups of polynomial growth and expanding maps", Inst. Hautes Études Sci. Publ. Math., 53:1 (1981), 53-78.

[46] B. Green, Notes on the polynomial Freiman--Ruzsa conjecture. http://people.maths.ox.ac.uk/greenbj/papers/PFR.pdf.

[47] I. Z. Ruzsa, "An analog of Freiman's theorem in groups", Structure theory of set addition, Astérisque, 258, Soc. Math. France, Paris, 1999, xv, 323-326.

[48] Y. Bilu, "Structure of sets with small sumset", Structure theory of sets addition, Astérisque, 258, Soc. Math. France, Paris, 1999, xi, 77-108.

[49] I. Z. Ruzsa, "Generalized arithmetical progressions and sumsets", Acta Math. Hungar., 65:4 (1994), 379-388.

[50] J. Bourgain, "Roth's theorem on progressions revisited", J. Anal. Math., 104:1 (2008), 155-192.

[51] B. Green, T. Tao, "A note on the Freiman and Balog-Szemerédi-Gowers theorems in finite fields", J. Aust. Math. Soc., 86:1 (2009), 61-74.

[52] B. Green, T. Tao, "Freiman's theorem in finite fields via extremal set theory", Combin. Probab. Comput., 18:3 (2009), 335-355.

[53] T. Sanders, Another proof of a Freiman-type theorem, 2007 (v2 - 2010), 11 pp., arXiv: $0710.2545 \mathrm{v} 1$.

[54] M.-C. Chang, "Some consequences of the polynomial Freiman-Ruzsa conjecture", C. R. Math. Acad. Sci. Paris, 347:11-12 (2009), 583-588.

[55] B. Green, T. Tao, "An equivalence between inverse sumset theorems and inverse conjectures for the $U^{3}$-norm", Math. Proc. Cambridge Philos. Soc., 149:1 (2010), $1-19$.

[56] B. Green, T. Tao, The Möbius and nilsequences conjecture, 2008 (v4 - 2011), 20 pp., arXiv: 0807.1736v1.

[57] B. Green, T. Tao, "The primes contain arbitrarily long arithmetic progressions", Ann. of Math. (2), 167:2 (2008), 481-547.

[58] Н. Г. Чудаков, "О плотности совокупности четных чисел, непредставимых как сумма двух нечетных простых", Изв. АН СССР. Сер. матем., 2:1 (1938), 25-40.

[59] J. G. van der Corput, "Über Summen von Primzahlen und Primzahlquadraten", Math. Ann., 116:1 (1939), 1-50.

[60] S. Chowla, "There exists an infinity of 3-combinations of primes in A.P.", Proc. Lahore Philos. Soc., 6:2 (1944), 15-16.

[61] E. Szemerédi, "On sets of integers containing no four elements in arithmetic progression", Acta Math. Acad. Sci. Hungar., 20:1-2 (1969), 89-104.

[62] E. Szemerédi, "On sets of integers containing no $k$ elements in arithmetic progression", Acta Arith., 27 (1975), 199-245.

[63] E. Szemerédi, V. Vu, "Long arithmetic progressions in sumsets: thresholds and bounds", J. Amer. Math. Soc., 19:1 (2006), 119-169.

[64] T. Tao, V. Vu, "On the singularity probability of random Bernoulli matrices", J. Amer. Math. Soc., 20:3 (2007), 603-628. 
[65] T. Schoen, I. D. Shkredov, "Roth's theorem in many variables", Israel J. Math., 199:1 (2014), 287-308.

[66] F.A. Behrend, "On sets of integers which contain no three terms in arithmetic progression", Proc. Natl. Acad. Sci. USA, 32:12 (1946), 331-332.

[67] J. Bourgain, "On aritmetic progressions in sums of sets of integers", A tribute to Paul Erdős, Cambridge Univ. Press, Cambridge, 1990, 105-109.

[68] I. Z. Ruzsa, "Arithmetic progressions in sumsets", Acta Arith., 60:2 (1991), 191-202.

[69] G. A. Freiman, H. Halberstam, I. Z. Ruzsa, "Integer sum sets containing long arithmetic progressions", J. Lond. Math. Soc. (2), 46:2 (1992), 193-201.

[70] J. Solymosi, "Arithmetic progressions in sets with small sumsets", Combin. Probab. Comput., 15:4 (2006), 597-603; 2005, 6 pp., arXiv: math/0503649.

[71] T. Sanders, "Additive structures in sumsets", Math. Proc. Cambridge Philos. Soc., 144:2 (2008), 289-316; 2006 (v2 - 2010), 26 pp., arXiv: math/0605520v1.

[72] B. Green, "Arithmetic progressions in sumsets", Geom. Funct. Anal., 12:3 (2002), 584-597.

[73] K. Henriot, "On arithmetic progressions in $A+B+C$ ", Int. Math. Res. Not. IMRN, 2014:18 (2014), 5134-5164.

[74] W. Rudin, "Trigonometric series with gaps", J. Math. Mech., 9 (1960), 203-227.

[75] S. V. Konyagin, I. Łaba, "Distance sets of well-distributed planar sets for polygonal norms", Israel J. Math., 152:1 (2006), 157-179.

[76] B. Green, T. Sanders, "A quantitative version of the idempotent theorem in harmonic analysis", Ann. of Math. (2), 168:3 (2008), 1025-1054.

[77] B. Green, T. Sanders, "Boolean functions with small spectral norm", Geom. Funct. Anal., 18:1 (2008), 144-162; 2006 (v2 - 2010), 17 pp., arXiv: math/0605524v1.

[78] T. Sanders, Indicator functions in the Fourier-Eymard algebra, 2009 (v2 - 2010), 76 pp., arXiv: 0912.0308v1.

[79] T. Sanders, An application of a local version of Chang's theorem, 2006, 13 pp., arXiv: math/0607668v1.

[80] S. V. Konyagin, I. D. Shkredov, On Wiener norm of subsets of $\mathbb{Z}_{p}$ of medium size, 2014, 15 c., arXiv: $1403.8129 \mathrm{v} 1$.

[81] N. Bogoliouboff, "Sur quelques propriétés arithmétiques des presque-périodes", Ann. Chaire Phys. Math. Kiev, 4 (1939), 185-205.

[82] T. Sanders, "A note on Freuman's theorem in vector spaces", Combin. Probab. Comput., 17:2 (2008), 297-305.

[83] T. Sanders, "Appendix to 'Roth's theorem on progressions revisited' by J. Bourgain", J. Anal. Math., 104 (2008), 193-206; 2007 (v1 - 2007, v4 - 2010 ), 11 pp., arXiv: $0710.0642 \mathrm{v} 2$.

[84] С. В. Конягин, "О теореме Фреймана в конечных полях", Матем. заметки, 84:3 (2008), 472-474; англ. пер.: S. V. Konyagin, "On the Freiman theorem in finite fields", Math. Notes, 84:3 (2008), 435-438.

[85] C. Even-Zohar, "On sums of generating sets in $\mathbb{Z}_{2}^{n}$ ", Combin. Probab. Comput., 21:6 (2012), 916-941; 2011 (v2 - 2012), 21 pp., arXiv: 1108.4902v1.

[86] C. Even-Zohar, S. Lovett, "The Freiman-Ruzsa theorem over finite fields", J. Combin. Theory Ser. A, 125 (2014), 333-341. 
[87] H. Plünnecke, Eigenschaften und Abschätzungen von Wirkungsfunktionen, Ber. Gesellsch. Math. Datenverarb., 22, Gesellschaft für Mathematik und Datenverarbeitung, Bonn, 1969, iii+173 pp.

[88] I. Z. Ruzsa, "An application of graph theory to additive number theory", Sci. Ser. A Math. Sci. (N. S.), 3 (1989), 97-109.

[89] G. Petridis, "New proofs of Plünnecke-type estimates for product sets in groups", Combinatorica, 32:6 (2012), 721-733.

[90] G. Petridis, "Plünnecke's inequality", Combin. Probab. Comput., 20:6 (2011), 921-938; 2011, 19 pp., arXiv: 1101.2532.

[91] I.Z. Ruzsa, "Sumsets and structure", Combinatorial number theory and additive group theory, Adv. Courses Math. CRM Barcelona, Birkhäuser Verlag, Basel, 2009, $87-210$.

[92] А. Г. Хованский, "Многогранник Ньютона, полином Гильберта и суммы конечных множеств", Функи. анализ и его прил., 26:4 (1992), 57-63; англ. пер.: A. G. Khovanskii, "Newton polyhedron, Hilbert polynomial, and sums of finite sets", Funct. Anal. Appl., 26:4 (1992), 276-281.

[93] M. B. Nathanson, I.Z. Ruzsa, "Polynomial growth of sumsets in abelian semigroups", J. Théor. Nombres Bordeaux, 14:2 (2002), 553-560; 2002, 8 pp., arXiv: math/0204052v1.

[94] T. Sanders, "On a theorem of Shkredov", Online J. Anal. Comb., 2010, № 5, 4 pp. (electronic only).

[95] T. Schoen, I. D. Shkredov, Additive dimension and a theorem of Sanders, 2014, 20 pp., arXiv: $1404.2044 \mathrm{v} 1$.

[96] T. Sanders, "On Roth's theorem on progressions", Ann. of Math. (2), 174:1 (2011), 619-636.

[97] А. А. Юдин, "О мере больших значений тригонометрических сумм", Теоретикочисловые исследования по спектру Маркова и структурной теории сложения множеств, ред. Г. А. Фрейман, А. М. Рубинов, Е. В. Новоселов, Калининский гос. ун-т, Калинин, 1973, 163-171.

[98] J. M. López, K. A. Ross, Sidon sets, Lecture Notes in Pure and Appl. Math., 13, Marcel Dekker, Inc., New York, 1975, v+193 pp.

[99] B. Green, "Spectral structure of sets of integers", Fourier analysis and convexity, Appl. Numer. Harmon. Anal., Birkhäuser Boston, Boston, MA, 2004, 83-96.

[100] B. Green, Structure theory of set addition, ICMS Instructional Conference in Combinatorial Aspects of Mathematical Analysis (Edinburgh), 2002, 27 pp.

[101] И. Д. Шкредов, "О множествах больших тригонометрических сумм", Изв. РАН. Сер. матем., 72:1 (2008), 161-182; англ. пер.: I. D. Shkredov, "On sets of large trigonometric sums", Izv. Math., 72:1 (2008), 149-168.

[102] И. Д. Шкредов, "Некоторые примеры множеств больших тригонометрических сумм", Матем. сб., 198:12 (2007), 105-140; англ. пер.: I. D. Shkredov, "Examples of sets with large trigonometric sums", Sb. Math., 198:12 (2007), 1805-1838.

[103] I. D. Shkredov, "On sumsets of dissociated sets", Online J. Anal. Comb., 2009, № 4, 26 pp. (electronic only).

[104] T.F. Bloom, A quantitative improvement of Roth's theorem on arithmetic progressions, 2014, 20 pp., arXiv: 1405.5800v1. 
[105] J. Bourgain, "On triples in arithmetic progression", Geom. Funct. Anal., 9:5 (1999), 968-984.

[106] J. Bourgain, N. Katz, T. Tao, "A sum-product estimate in finite fields, and applications", Geom. Funct. Anal., 14:1 (2004), 27-57.

[107] M.-C. Chang, "The Erdős-Szemerédi problem on sum set and product set", Ann. of Math. (2), 157:3 (2003), 939-957.

[108] M.-C. Chang, "On problems of Erdös and Rudin", J. Funct. Anal., 207:2 (2004), 444-460.

[109] T. Schoen, "New bounds in Balog-Szemerédi-Gowers theorem", Combinatorica, 34:5 (2014), 7 pp.; www.staff.amu.edu.pl/ ${ }^{\sim}$ schoen/remark-B-S-G.pdf.

[110] J. Bourgain, M.Z. Garaev, "On a variant of sum-product estimates and explicit exponential sum bounds in prime fields", Math. Proc. Cambridge Philos. Soc., 146:1 (2009), 1-21.

[111] P. Erdős, E. Szemerédi, "On sums and products of integers", Studies in pure mathematics, ed. P. Erdős, Birkhäuser, Basel, 1983, 213-218.

[112] G. Elekes, "On the number of sums and products", Acta Arith., 81:4 (1997), 365-367.

[113] J. Solymosi, "Bounding multiplicative energy by the sumset", Adv. Math., 222:2 (2009), 402-408.

[114] J. Bourgain, A. A. Glibichuk, S. V. Konyagin, "Estimate for the number of sums and products and for exponential sums in fields of prime order", J. Lond. Math. Soc. (2), 73:2 (2006), 380-398.

[115] B. Green, Sum-product phenomena in $\mathbb{F}_{p}$ : a brief introduction, 2009, 10 pp., arXiv: $0904.2075 \mathrm{v} 1$.

[116] A. A. Glibichuk, "Average estimate for additive energy in prime field", Mosc. J. Comb. Number Theory, 1:3 (2011), 50-68.

[117] I. D. Shkredov, "On Heilbronn's exponential sum”, Q. J. Math., 64:4 (2013), 1221-1230.

[118] I. D. Shkredov, "Some new inequalities in additive combinatorics", Mosc. J. Comb. Number Theory, 3:3-4 (2013), 189-239.

[119] I. D. Shkredov, "On exponential sums over multiplicative subgroups of medium size", Finite Fields Appl., 30 (2014), 72-87.

[120] D. R. Heath-Brown, S. Konyagin, "New bounds for Gauss sums derived from $k$ th powers, and for Heilbronn's exponential sum", Q. J. Math., 51:2 (2000), 221-235.

[121] С. В. Конягин, "Оценки тригонометрических сумм по подгруппам и сумм Гаусса", IV Международная конферениия "Современные проблемы теории чисел и ее приложения”. Актуалъные проблемы, Ч. 3 (Тула, 2001), МГУ, М., 2002, 86-114.

[122] D. R. Heath-Brown, "An estimate for Heilbronn's exponential sum", Analytic number theory, v. 2 (Allerton Park, IL, 1995), Progr. Math., 139, Birkhäuser Boston, Boston, MA, 1996, 451-463.

[123] J. Bourgain, K. Ford, S. V. Konyagin, I. E. Shparlinski, "On the divisibility of Fermat quotients", Michigan Math. J., 59:2 (2010), 313-328.

[124] B. Host, B. R. Kra, "Nonconventional ergodic averages and nilmanifolds", Ann. of Math. (2), 161:1 (2005), 397-488.

[125] B. Host, B. Kra, "Convergence of polynomial ergodic averages", Israel J. Math., 149:1 (2005), 1-19. 
[126] T. Tao, "A quantitative ergodic theory proof of Szemerédi's theorem", Electron. J. Combin., 13:1 (2006), R99, 49 pp.

[127] T. Tao, "Norm convergence of multiple ergodic averages for commuting transformations", Ergodic Theory Dynam. Systems, 28:2 (2008), 657-688.

[128] T. Tao, T. Ziegler, "The inverse conjecture for the Gowers norm over finite fields via the correspondence principle", Anal. PDE, 3:1 (2010), 1-20.

[129] T. Ziegler, "A non-conventional ergodic theorem for a nilsystem", Ergodic Theory Dynam. Systems, 25:4 (2005), 1357-1370.

[130] T. Ziegler, "Universal characteristic factors and Furstenberg averages", J. Amer. Math. Soc., 20:1 (2007), 53-97 (electronic).

[131] K. F. Roth, "On certain sets of integers", J. London Math. Soc., 28 (1953), 104-109.

[132] E. Szemerédi, "Regular partitions of graphs", Problèmes combinatoires et théorie des graphes (Univ. Orsay, Orsay, 1976), Colloq. Internat. CNRS, 260, CNRS, Paris, 1978, 399-401.

[133] A. Sárközy, "On additive decompositions of the set of quadratic residues modulo $p$ ", Acta Arith., 155 (2012), 41-51.

[134] C. Dartyge, A. Sárközy, "On additive decompositions of the set of primitive roots modulo p", Monatsh. Math., 169:3-4 (2013), 317-328.

[135] N. H. Katz, P. Koester, "On additive doubling and energy", SIAM J. Discrete Math., 24:4 (2010), 1684-1693.

[136] P. Erdös, P. Turán, "On some sequences of integers", J. London Math. Soc., 11:4 (1936), 261-264.

[137] B. L. van der Waerden, "Beweis einer Baudetschen Vermutung", Nieuw Arch. Wisk., 15 (1927), 212-216.

[138] E. Szemerédi, "Integer sets containing no arithmetic progressions", Acta Math. Hungar., 56:1-2 (1990), 155-158.

[139] D. R. Heath-Brown, "Integer sets containing no arithmetic progressions", J. London Math. Soc. (2), 35:3 (1987), 385-394.

[140] R. Meshulam, "On subsets of finite abelian groups with no 3-term arithmetic progressions", J. Combin. Theory Ser. A, 71:1 (1995), 168-172.

[141] H. Furstenberg, Recurrence in ergodic theory and combinatorial number theory, M. B. Porter Lectures, Princeton Univ. Press, Princeton, N.J., 1981, xi+203 pp.

[142] T. Austin, "On the norm convergence of non-conventional ergodic averages", Ergodic Theory Dynam. Systems, 30:2 (2010), 321-338; 2008 (v3 - 2009), 19 pp., arXiv: 0805.0320v1.

[143] W. T. Gowers, "Hypergraph regularity and the multidimensional Szemerédi theorem", Ann. of Math. (2), 166:3 (2007), 897-946.

[144] B. Nagle, V. Rödl, M. Schacht, "The counting lemma for regular $k$-uniform hypergraphs", Random Structures Algorithms, 28:2 (2006), 113-179.

[145] V. Rödl, J. Skokan, "Regularity lemma for k-uniform hypergraphs", Random Structures Algorithms, 25:1 (2004), 1-42.

[146] T. Tao, "A variant of the hypergraph removal lemma", J. Combin. Theory Ser. A, 113:7 (2006), 1257-1280.

[147] T. Sanders, "Green's sumset problem at density one half", Acta Arith., 146:1 (2011), 91-101. 
[148] Р. Грэхем, Начала теории Рамсея, Мир, М., 1984, 97 с.; пер.с англ.: R. L. Graham, Rudiments of Ramsey theory, CBMS Reg. Conf. Ser. Math., 45, Providence, RI, 1981, v+65 pp.

[149] J. Bourgain, "More on the sum-product phenomenon in prime fields and its applications", Int. J. Number Theory, 1:1 (2005), 1-32.

\section{Илья Дмитриевич Шкредов} (Ilya D. Shkredov )

Математический институт им. В. А. Стеклова РАН;

Институт проблем передачи информации

им. А. А. Харкевича РАН

E-mail: ilya.shkredov@gmail.com 